Centers for Disease Control and Prevention

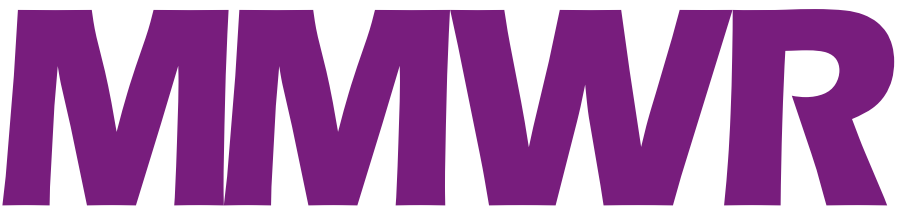

Morbidity and Mortality Weekly Report

Recommendations and Reports / Vol. 65 / No. 4 July 29, 2016

\title{
U.S. Selected Practice Recommendations for Contraceptive Use, 2016
}

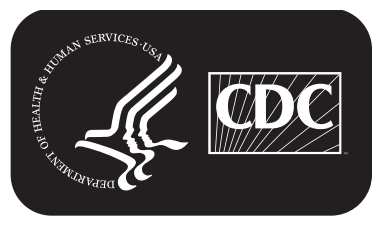




\section{CONTENTS}

Introduction . 1

Summary of Changes from the

2013 U.S. SPR . .2

Methods .. .2

Maintaining Updated Guidance .................................................................. 3

How To Use This Document............................................................................

Contraceptive Method Choice ...................................................................... 4

How To Be Reasonably Certain that a Woman Is Not Pregnant.............5

Intrauterine Contraception ............................................................................. 7

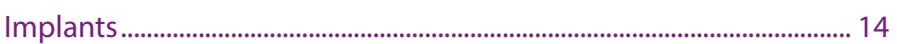

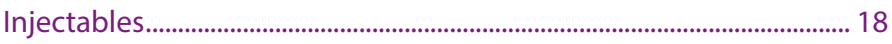

Combined Hormonal Contraceptives ........................................................ 22

Progestin-Only Pills........................................................................................ 31

Standard Days Method.................................................................................. 34

Emergency Contraception ........................................................................ 34

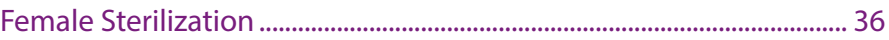

Male Sterilization ...................................................................................................... 37

When Women Can Stop Using Contraceptives ......................................... 38

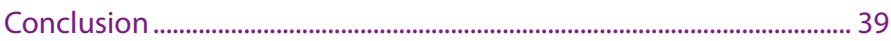

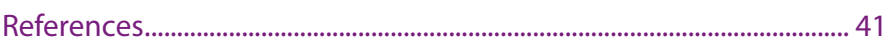

\section{CONTENTS (Continued)}

Appendix A: Summary Chart of U.S. Medical Eligibility Criteria for

Contraceptive Use, 2016 53

Appendix B: When To Start Using Specific Contraceptive Methods ....... 62

Appendix C: Examinations and Tests Needed Before Initiation of

Contraceptive Methods 63

Appendix D: Routine Follow-Up After Contraceptive Initiation......... 64

Appendix E: Management of Women with Bleeding Irregularities

While Using Contraception 65

Appendix F: Management of Intrauterine Devices When Users are

Found To Have Pelvic Inflammatory Disease 66

\section{Disclosure of Relationship}

CDC, our planners, and our content experts wish to disclose they have no financial interest or other relationships with the manufacturers of commercial products, suppliers of commercial services, or commercial supporters. Planners have reviewed content to ensure there is no bias. This document will not include any discussion of the unlabeled use of a product or a product under investigational use, with the exception that some of the recommendations in this document might be inconsistent with package labeling.

The MMWR series of publications is published by the Center for Surveillance, Epidemiology, and Laboratory Services, Centers for Disease Control and Prevention (CDC), U.S. Department of Health and Human Services, Atlanta, GA 30329-4027.

Suggested citation: [Author names; first three, then et al., if more than six.] [Title]. MMWR Recomm Rep 2016;65(No. RR-\#):[inclusive page numbers].

\section{Centers for Disease Control and Prevention}

Thomas R. Frieden, MD, MPH, Director

Harold W. Jaffe, MD, MA, Associate Director for Science

Joanne Cono, MD, ScM, Director, Office of Science Quality

Chesley L. Richards, MD, MPH, Deputy Director for Public Health Scientific Services

Michael F. Iademarco, MD, MPH, Director, Center for Surveillance, Epidemiology, and Laboratory Services

\section{MMWR Editorial and Production Staff (Serials)}

Sonja A. Rasmussen, MD, MS, Editor-in-Chief

Charlotte K. Kent, PhD, MPH, Executive Editor

Christine G. Casey, MD, Editor

Teresa F. Rutledge, Managing Editor

David C. Johnson, Lead Technical Writer-Editor

Catherine B. Lansdowne, MS, Project Editor
Martha F. Boyd, Lead Visual Information Specialist

Maureen A. Leahy, Julia C. Martinroe,

Stephen R. Spriggs, Moua Yang, Tong Yang, Visual Information Specialists

Quang M. Doan, MBA, Phyllis H. King, Terraye M. Starr,

Information Technology Specialists

\section{MMWR Editorial Board}

Timothy F. Jones, MD, Chairman

Matthew L. Boulton, MD, MPH

Virginia A. Caine, MD

Katherine Lyon Daniel, PhD

Jonathan E. Fielding, MD, MPH, MBA

David W. Fleming, MD
William E. Halperin, MD, DrPH, MPH

King K. Holmes, MD, PhD

Robin Ikeda, MD, MPH

Rima F. Khabbaz, MD

Phyllis Meadows, PhD, MSN, RN

Jewel Mullen, MD, MPH, MPA
Jeff Niederdeppe, $\mathrm{PhD}$

Patricia Quinlisk, MD, MPH

Patrick L. Remington, MD, MPH Carlos Roig, MS, MA

William L. Roper, MD, MPH

William Schaffner, MD 


\title{
U.S. Selected Practice Recommendations for Contraceptive Use, 2016
}

\author{
Kathryn M. Curtis, $\mathrm{PhD}^{1}$ \\ Tara C. Jatlaoui, $\mathrm{MD}^{1}$ \\ Naomi K. Tepper, $\mathrm{MD}^{1}$ \\ Lauren B. Zapata, $\mathrm{PhD}^{1}$ \\ Leah G. Horton, MSPH${ }^{1}$ \\ Denise J. Jamieson, $\mathrm{MD}^{1}$ \\ Maura K. Whiteman, $\mathrm{PhD}^{1}$ \\ ${ }^{1}$ Division of Reproductive Health, National Center for Chronic Disease Prevention and Health Promotion
}

\section{Summary}

\begin{abstract}
The 2016 U.S. Selected Practice Recommendations for Contraceptive Use (U.S. SPR) addresses a select group of common, yet sometimes controversial or complex, issues regarding initiation and use of specific contraceptive methods. These recommendations for health care providers were updated by CDC after review of the scientific evidence and consultation with national experts who met in Atlanta, Georgia, during August 26-28, 2015. The information in this report updates the 2013 U.S. SPR (CDC. U.S. selected practice recommendations for contraceptive use, 2013. MMWR 2013;62[No. RR-5]). Major updates include 1) revised recommendations for starting regular contraception after the use of emergency contraceptive pills and 2) new recommendations for the use of medications to ease insertion of intrauterine devices. The recommendations in this report are intended to serve as a source of clinical guidance for health care providers and provide evidence-based guidance to reduce medical barriers to contraception access and use. Health care providers should always consider the individual clinical circumstances of each person seeking family planning services. This report is not intended to be a substitute for professional medical advice for individual patients. Persons should seek advice from their health care providers when considering family planning options.
\end{abstract}

\section{Introduction}

Unintended pregnancy rates remain high in the United States; approximately $45 \%$ of all pregnancies are unintended, with higher proportions among adolescent and young women, women who are racial/ethnic minorities, and women with lower levels of education and income (1). Unintended pregnancies increase the risk for poor maternal and infant outcomes (2) and in 2010, resulted in U.S. government health care expenditures of $\$ 21$ billion (3). Approximately half of unintended pregnancies are among women who were not using contraception at the time they became pregnant; the other half are among women who became pregnant despite reported use of contraception (4). Strategies to prevent unintended pregnancy include assisting women at risk for unintended pregnancy and their partners with choosing appropriate contraceptive methods and helping them use methods correctly and consistently to prevent pregnancy.

In 2013, CDC published the first U.S. Selected Practice Recommendations for Contraceptive Use (U.S. SPR), adapted from global guidance developed by the World Health Organization (WHO SPR), which provided evidence-based guidance on how to use contraceptive methods safely and effectively once they are deemed to be medically appropriate.

Corresponding author: Kathryn M. Curtis, PhD, Division of Reproductive Health, National Center for Chronic Disease Prevention and Health Promotion, CDC. Telephone: 770-488-5200; E-mail: kmc6@cdc.gov.
U.S. SPR is a companion document to U.S. Medical Eligibility Criteria for Contraceptive Use (U.S. MEC) (http://www.cdc. gov/reproductivehealth/unintendedpregnancy/usmec.htm), which provides recommendations on safe use of contraceptive methods for women with various medical conditions and other characteristics (5). WHO intended for the global guidance to be used by local or national policy makers, family planning program managers, and the scientific community as a reference when they develop family planning guidance at the country or program level. During 2012-2013, CDC went through a formal process to adapt the global guidance for best implementation in the United States, which included rigorous identification and critical appraisal of the scientific evidence through systematic reviews, and input from national experts on how to translate that evidence into recommendations for U.S. health care providers ( 6 ). At that time, CDC committed to keeping this guidance up to date and based on the best available evidence, with full review every few years ( $($ ).

This document updates the 2013 U.S. SPR (6) with new evidence and input from experts. Major updates include 1) revised recommendations for starting regular contraception after the use of emergency contraceptive pills and 2) new recommendations for the use of medications to ease insertion of intrauterine devices (IUDs). Recommendations are provided for health care providers on the safe and effective use of contraceptive methods and address provision of contraceptive methods and management of side effects and other problems 
with contraceptive method use, within the framework of removing unnecessary medical barriers to accessing and using contraception. These recommendations are meant to serve as a source of clinical guidance for health care providers; health care providers should always consider the individual clinical circumstances of each person seeking family planning services. This report is not intended to be a substitute for professional medical advice for individual patients, who should seek advice from their health care providers when considering family planning options.

\section{Summary of Changes from the 2013 U.S. SPR}

\section{Updated Recommendations}

Recommendations have been updated regarding when to start regular contraception after ulipristal acetate (UPA) emergency contraceptive pills:

- Advise the woman to start or resume hormonal contraception no sooner than 5 days after use of UPA, and provide or prescribe the regular contraceptive method as needed. For methods requiring a visit to a health care provider, such as depo-medroxyprogesterone acetate (DMPA), implants, and IUDs, starting the method at the time of UPA use may be considered; the risk that the regular contraceptive method might decrease the effectiveness of UPA must be weighed against the risk of not starting a regular hormonal contraceptive method.

- The woman needs to abstain from sexual intercourse or use barrier contraception for the next 7 days after starting or resuming regular contraception or until her next menses, whichever comes first.

- Any nonhormonal contraceptive method can be started immediately after the use of UPA.

- Advise the woman to have a pregnancy test if she does not have a withdrawal bleed within 3 weeks.

\section{New Recommendations}

New recommendations have been made for medications to ease IUD insertion:

- Misoprostol is not recommended for routine use before IUD insertion. Misoprostol might be helpful in select circumstances (e.g., in women with a recent failed insertion).

- Paracervical block with lidocaine might reduce patient pain during IUD insertion.

\section{Methods}

Since publication of the 2013 U.S. SPR, CDC has monitored the literature for new evidence relevant to the recommendations through the $\mathrm{WHO} / \mathrm{CDC}$ continuous identification of research evidence (CIRE) system (7). This system identifies new evidence as it is published and allows WHO and CDC to update systematic reviews and facilitate updates to recommendations as new evidence warrants. Automated searches are run in PubMed weekly, and the results are reviewed. Abstracts that meet specific criteria are added to the web-based CIRE system, which facilitates coordination and peer review of systematic reviews for both WHO and CDC. In 2014, CDC reviewed all of the existing recommendations in the 2013 U.S. SPR for new evidence identified by CIRE that had the potential to lead to a changed recommendation. During August 27-28, 2014, CDC held a meeting in Atlanta, Georgia, of 11 family planning experts and representatives from partner organizations to solicit their input on the scope of and process for updating both the 2010 U.S. MEC and the 2013 U.S. SPR. The participants were experts in family planning and represented different provider types and organizations that represent health care providers. A list of participants is provided at the end of this report. The meeting related to topics to be addressed in the update of U.S. SPR based on new scientific evidence published since 2013 (identified though the CIRE system), topics addressed at a $2014 \mathrm{WHO}$ meeting to update global guidance, and suggestions $\mathrm{CDC}$ received from providers for the addition of recommendations not included in the 2013 U.S. SPR (e.g., from provider feedback through e-mail, public inquiry, and questions received at conferences). CDC identified one topic to consider adding to the guidance: the use of medications to ease IUD insertion (evidence question: "Among women of reproductive age, does use of medications before IUD insertion improve the safety or effectiveness of the procedure [ease of insertion, need for adjunctive insertion measures, or insertion success] or affect patient outcomes [pain or side effects] compared with nonuse of these medications?"). CDC also identified one topic for which new evidence warranted a review of an existing recommendation: initiation of regular contraception after emergency contraceptive pills (evidence question: "Does ulipristal acetate for emergency contraception interact with regular use of hormonal contraception leading to decreased effectiveness of either contraceptive method?"). CDC determined that all other recommendations in the 2013 U.S. SPR were up to date and consistent with the current body of evidence for that recommendation.

In preparation for a subsequent expert meeting August 26-28, 2015, to review the scientific evidence 
for potential recommendations, CDC staff conducted independent systematic reviews for each of the topics being considered. The purpose of these systematic reviews was to identify direct evidence related to the common clinical challenges associated with the recommendations. Preferred Reporting Items for Systematic Reviews and Meta-Analyses (PRISMA) guidelines were followed for reporting systematic reviews $(8,9)$, and strength and quality of the evidence were assigned using the system of the U.S. Preventive Services Task Force (10). When direct evidence was limited or not available, indirect evidence (e.g., evidence on surrogate outcomes) and theoretical issues were considered and either added to direct evidence within a systematic review or separately compiled for presentation to the meeting participants. Completed systematic reviews were peer reviewed by two or three experts and then provided to participants before the expert meeting. Reviews are referenced throughout this document; the full reviews have been published and contain the details of each review, including systematic review question, literature search protocol, inclusion and exclusion criteria, evidence tables, and quality assessment. CDC staff continued to monitor new evidence identified through the CIRE system during the preparation for the August 2015 meeting.

During August 26-28, 2015, CDC held a meeting in Atlanta, Georgia, of 29 participants who were invited to provide their individual perspectives on the scientific evidence presented and to discuss potential recommendations that followed. Participants represented a wide range of expertise in family planning provision and research and included obstetrician/gynecologists, pediatricians, family physicians, nurse practitioners, epidemiologists, and others with research and clinical practice expertise in contraceptive safety, effectiveness, and management. Lists of participants and any potential conflicts of interest are provided at the end of this report. During the meeting, the evidence from the systematic review for each topic was presented, including direct evidence and any indirect evidence or theoretical concerns. Participants provided their perspectives on using the evidence to develop the recommendations that would meet the needs of U.S. health care providers. After the meeting, CDC determined the recommendations in this report, taking into consideration the perspectives provided by the meeting participants. Feedback also was received from four external reviewers, composed of health care providers and researchers who had not participated in the update meetings. These providers were asked to provide comments on the accuracy, feasibility, and clarity of the recommendations. Areas of research that need additional investigation also were considered during the meeting (11).

\section{Maintaining Updated Guidance}

As with any evidence-based guidance document, a key challenge is keeping the recommendations up to date as new scientific evidence becomes available. Working with WHO, CDC uses the CIRE system to ensure that WHO and CDC guidance is based on the best available evidence and that a mechanism is in place to update guidance when new evidence becomes available (7). CDC will continue to work with WHO to identify and assess all new relevant evidence and determine whether changes in the recommendations are warranted. In most cases, U.S. SPR will follow any updates in the WHO guidance, which typically occurs every 5 years (or sooner if warranted by new data). In addition, CDC will review any interim WHO updates for their application in the United States. CDC also will identify and assess any new literature for the recommendations that are not included in the WHO guidance and will completely review U.S. SPR every 5 years. Updates to the guidance can be found on the U.S. SPR website (http://www.cdc.gov/reproductivehealth/ UnintendedPregnancy/USSPR.htm).

\section{How To Use This Document}

The recommendations in this report are intended to help health care providers address issues related to use of contraceptives, such as how to help a woman initiate use of a contraceptive method, which examinations and tests are needed before initiating use of a contraceptive method, what regular follow-up is needed, and how to address problems that often arise during use, including missed pills and side effects such as unscheduled bleeding. Each recommendation addresses what a woman or health care provider can do in specific situations. For situations in which certain groups of women might be medically ineligible to follow the recommendations, comments and reference to U.S. MEC are provided (5). The full U.S. MEC recommendations and the evidence supporting those recommendations have been updated in 2016 (5) and are summarized (Appendix A).

The information in this document is organized by contraceptive method, and the methods generally are presented in order of effectiveness, from highest to lowest. However, the recommendations are not intended to provide guidance on every aspect of provision and management of contraceptive method use. Instead, they incorporate the best available evidence to address specific issues regarding common, yet sometimes complex, clinical issues. Each contraceptive method section generally includes information about initiation of the method, regular follow-up, and management of problems with use (e.g., usage errors and side effects). Each section first 
provides the recommendation and then includes comments and a brief summary of the scientific evidence on which the recommendation is based. The level of evidence from the systematic reviews for each evidence summary are provided based on the U.S. Preventive Services Task Force system, which includes ratings for study design (I: randomized controlled trials; II-1: controlled trials without randomization; II-2: observational studies; and II-3: multiple time series or descriptive studies), ratings for internal validity (good, fair, or poor), and categorization of the evidence as direct or indirect for the specific review question (10).

Recommendations in this document are provided for permanent methods of contraception, such as vasectomy and female sterilization, as well as for reversible methods of contraception, including the copper-containing intrauterine device (Cu-IUD); levonorgestrel-releasing IUDs (LNG-IUDs); the etonogestrel implant; progestin-only injectables; progestinonly pills (POPs); combined hormonal contraceptive methods that contain both estrogen and a progestin, including combined oral contraceptives (COCs), a transdermal contraceptive patch, and a vaginal contraceptive ring; and the standard days method (SDM). Recommendations also are provided for emergency use of the Cu-IUD and emergency contraceptive pills (ECPs).

For each contraceptive method, recommendations are provided on the timing for initiation of the method and indications for when and for how long additional contraception, or a back-up method, is needed. Many of these recommendations include guidance that a woman can start a contraceptive method at any time during her menstrual cycle if it is reasonably certain that she is not pregnant. Guidance for health care providers on how to be reasonably certain that a woman is not pregnant also is provided.

For each contraceptive method, recommendations include the examinations and tests needed before initiation of the method. These recommendations apply to persons who are presumed to be healthy. Those with known medical problems or other special conditions might need additional examinations or tests before being determined to be appropriate candidates for a particular method of contraception. U.S. MEC might be useful in such circumstances (5). Most women need no or very few examinations or tests before initiating a contraceptive method although they might be needed to address other noncontraceptive health needs (12). Any additional screening needed for preventive health care can be performed at the time of contraception initiation, and initiation should not be delayed for test results. The following classification system was developed by WHO and adopted by CDC to categorize the applicability of the various examinations or tests before initiation of contraceptive methods (13):

Class A: These tests and examinations are essential and mandatory in all circumstances for safe and effective use of the contraceptive method.
Class B: These tests and examinations contribute substantially to safe and effective use, although implementation can be considered within the public health context, service context, or both. The risk for not performing an examination or test should be balanced against the benefits of making the contraceptive method available.

Class C: These tests and examinations do not contribute substantially to safe and effective use of the contraceptive method.

These classifications focus on the relation of the examinations or tests to safe initiation of a contraceptive method. They are not intended to address the appropriateness of these examinations or tests in other circumstances. For example, some of the examinations or tests that are not deemed necessary for safe and effective contraceptive use might be appropriate for good preventive health care or for diagnosing or assessing suspected medical conditions. Systematic reviews were conducted for several different types of examinations and tests to assess whether a screening test was associated with safe use of contraceptive methods. Because no single convention exists for screening panels for certain diseases, including diabetes, lipid disorders, and liver diseases, the search strategies included broad terms for the tests and diseases of interest.

Summary charts and clinical algorithms that summarize the guidance for the various contraceptive methods have been developed for many of the recommendations, including when to start using specific contraceptive methods (Appendix B), examinations and tests needed before initiating the various contraceptive methods (Appendix C), routine follow-up after initiating contraception (Appendix D), management of bleeding irregularities (Appendix E), and management of IUDs when users are found to have pelvic inflammatory disease (PID) (Appendix F). These summaries might be helpful to health care providers when managing family planning patients. Additional tools are available on the U.S. SPR website (http://www.cdc. gov/reproductivehealth/UnintendedPregnancy/USSPR.htm).

\section{Contraceptive Method Choice}

Many elements need to be considered individually by a woman, man, or couple when choosing the most appropriate contraceptive method. Some of these elements include safety, effectiveness, availability (including accessibility and affordability), and acceptability. Although most contraceptive methods are safe for use by most women, U.S. MEC provides recommendations on the safety of specific contraceptive methods for women with certain characteristics and medical conditions (5); a U.S. MEC summary (Appendix A) and the categories of medical eligibility criteria for contraceptive use (Box 1) are provided. 
Voluntary informed choice of contraceptive methods is an essential guiding principle, and contraceptive counseling, where applicable, might be an important contributor to the successful use of contraceptive methods.

Contraceptive method effectiveness is critically important in minimizing the risk for unintended pregnancy, particularly

\section{BOX 1. Categories of medical eligibility criteria for contraceptive use}

- U.S. MEC 1 = A condition for which there is no restriction for the use of the contraceptive method.

- U.S. MEC 2 = A condition for which the advantages of using the method generally outweigh the theoretical or proven risks.

- U.S. MEC 3 = A condition for which the theoretical or proven risks usually outweigh the advantages of using the method.

- U.S. MEC 4 = A condition that represents an unacceptable health risk if the contraceptive method is used.

Source: Curtis KM, Jatlaoui TC, Tepper NK, et al. U.S. medical eligibility criteria for contraceptive use. MMWR 2016;65(No. RR-3).

Abbreviation: U.S. MEC $=$ U.S. Medical Eligibility Criteria for

Contraceptive Use.

among women for whom an unintended pregnancy would pose additional health risks. The effectiveness of contraceptive methods depends both on the inherent effectiveness of the method itself and on how consistently and correctly it is used (Figure 1). Both consistent and correct use can vary greatly with characteristics such as age, income, desire to prevent or delay pregnancy, and culture. Methods that depend on consistent and correct use by clients have a wide range of effectiveness between typical use (actual use, including incorrect or inconsistent use) and perfect use (correct and consistent use according to directions) (14). IUDs and implants are considered longacting, reversible contraception (LARC); these methods are highly effective because they do not depend on regular compliance from the user. LARC methods are appropriate for most women, including adolescents and nulliparous women. All women should be counseled about the full range and effectiveness of contraceptive options for which they are medically eligible so that they can identify the optimal method.

In choosing a method of contraception, dual protection from the simultaneous risk for human immunodeficiency virus (HIV) and other sexually transmitted diseases (STDs) also should be considered. Although hormonal contraceptives and IUDs are highly effective at preventing pregnancy, they do not protect against STDs, including HIV. Consistent and correct use of the male latex condom reduces the risk for HIV infection and other STDs, including chlamydial infection, gonococcal infection, and trichomoniasis (15). Although evidence is limited, use of female condoms can provide protection from acquisition and transmission of STDs (15). All patients, regardless of contraceptive choice, should be counseled about the use of condoms and the risk for STDs, including HIV infection (15). Additional information about prevention and treatment of STDs is available from the CDC Sexually Transmitted Diseases Treatment Guidelines (http://www. cdc.gov/std/treatment) (15).

Women, men, and couples have increasing numbers of safe and effective choices for contraceptive methods, including LARC methods such as IUDs and implants, to reduce the risk for unintended pregnancy. However, with these expanded options comes the need for evidence-based guidance to help health care providers offer quality family planning care to their patients, including assistance in choosing the most appropriate contraceptive method for individual circumstances and using that method correctly, consistently, and continuously to maximize effectiveness. Removing unnecessary barriers can help patients access and successfully use contraceptive methods. Several medical barriers to initiating and continuing contraceptive methods might exist, such as unnecessary screening examinations and tests before starting the method (e.g., a pelvic examination before initiation of COCs), inability to receive the contraceptive on the same day as the visit (e.g., waiting for test results that might not be needed or waiting until the woman's next menstrual cycle to start use), and difficulty obtaining continued contraceptive supplies (e.g., restrictions on number of pill packs dispensed at one time). Removing unnecessary steps, such as providing prophylactic antibiotics at the time of IUD insertion or requiring unnecessary follow-up procedures, also can help patients access and successfully use contraception.

\section{How To Be Reasonably Certain that a Woman Is Not Pregnant}

In most cases, a detailed history provides the most accurate assessment of pregnancy risk in a woman who is about to start using a contraceptive method. Several criteria for assessing pregnancy risk are listed in the recommendation that follows. These criteria are highly accurate (i.e., a negative predictive value of $99 \%-100 \%)$ in ruling out pregnancy among women who are not pregnant (16-19). Therefore, CDC recommends that health care providers use these criteria to assess pregnancy status in a woman who is about to start using contraceptives (Box 2). If a woman meets one of these criteria (and therefore the health care provider can be reasonably certain that she is not pregnant), a urine pregnancy 
FIGURE 1. Effectiveness of family planning methods*

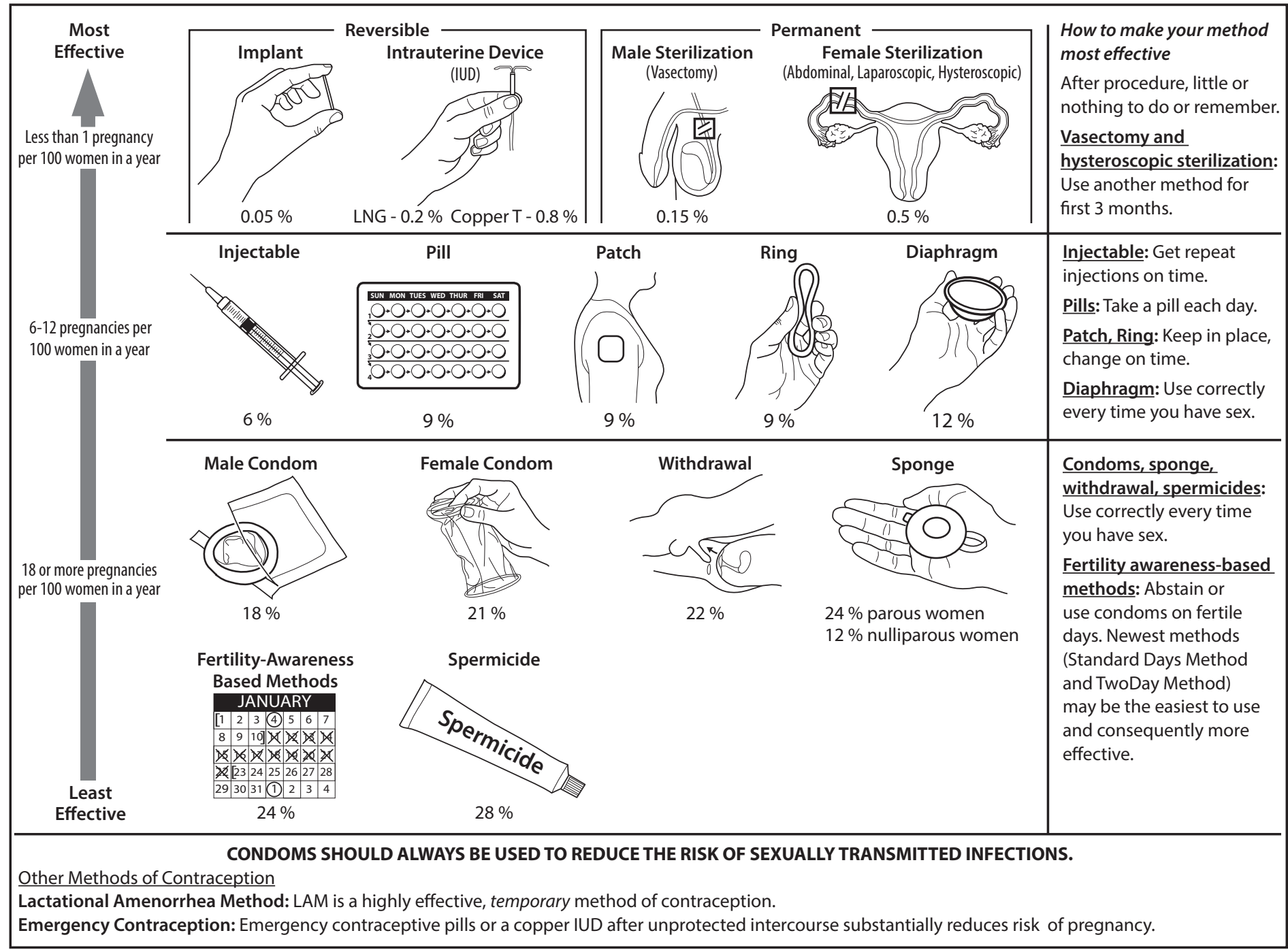

Sources: Adapted from World Health Organization (WHO) Department of Reproductive Health and Research, Johns Hopkins Bloomberg School of Public Health/ Center for Communication Programs (CCP). Knowledge for health project. Family planning: a global handbook for providers (2011 update). Baltimore, MD; Geneva, Switzerland: CCP and WHO; 2011; and Trussell J. Contraceptive failure in the United States. Contraception 2011;83:397-404.

* The percentages indicate the number out of every 100 women who experienced an unintended pregnancy within the first year of typical use of each contraceptive method.

test might be considered in addition to these criteria (based on clinical judgment), bearing in mind the limitations of the accuracy of pregnancy testing. If a woman does not meet any of these criteria, then the health care provider cannot be reasonably certain that she is not pregnant, even with a negative pregnancy test. Routine pregnancy testing for every woman is not necessary.

On the basis of clinical judgment, health care providers might consider the addition of a urine pregnancy test; however, they should be aware of the limitations, including accuracy of the test relative to the time of last sexual intercourse, recent delivery, or spontaneous or induced abortion. Routine pregnancy testing for every woman is not necessary. If a woman has had recent (i.e., within the last 5 days) unprotected sexual intercourse, consider offering emergency contraception (either a Cu-IUD or ECPs) if pregnancy is not desired.

Comments and Evidence Summary. The criteria for determining whether a woman is pregnant depend on the assurance that she has not ovulated within a certain amount of time after her last menses, spontaneous or induced abortion, or delivery. Among menstruating women, the timing of ovulation can vary widely. During an average 28 -day cycle, ovulation generally occurs during days 9-20 (20). In addition, the likelihood of ovulation is low from days $1-7$ of the menstrual cycle (21). After a spontaneous or an induced abortion, ovulation can occur within 2-3 weeks and has been found to occur as early as $8-13$ days after the end of the pregnancy. Therefore, the likelihood of ovulation is low $\leq 7$ days after 
BOX 2. How to be reasonably certain that a woman is not pregnant

A health care provider can be reasonably certain that a woman is not pregnant if she has no symptoms or signs of pregnancy and meets any one of the following criteria:

- is $\leq 7$ days after the start of normal menses

- has not had sexual intercourse since the start of last normal menses.

- has been correctly and consistently using a reliable method of contraception

- is $\leq 7$ days after spontaneous or induced abortion

- is within 4 weeks postpartum

- is fully or nearly fully breastfeeding (exclusively breastfeeding or the vast majority $[\geq 85 \%]$ of feeds are breastfeeds), amenorrheic, and $<6$ months postpartum

an abortion (22-24). A systematic review reported that the mean day of first ovulation among postpartum nonlactating women occurred 45-94 days after delivery (25). In one study, the earliest ovulation was reported at 25 days after delivery. Among women who are within 6 months postpartum, are fully or nearly fully breastfeeding (exclusively breastfeeding or the vast majority $[\geq 85 \%]$ of feeds are breastfeeds), and are amenorrheic, the risk for pregnancy is $<2 \%(26,27)$.

Although pregnancy tests often are performed before initiating contraception, the accuracy of qualitative urine pregnancy tests varies depending on the timing of the test relative to missed menses, recent sexual intercourse, or recent pregnancy. The sensitivity of a pregnancy test is defined as the concentration of human chorionic gonadotropin (hCG) at which $95 \%$ of tests are positive. Most qualitative pregnancy tests approved by the U.S. Food and Drug Administration (FDA) report a sensitivity of $20-25 \mathrm{mIU} / \mathrm{mL}$ in urine $(28-31)$. However, pregnancy detection rates can vary widely because of differences in test sensitivity and the timing of testing relative to missed menses $(30,32)$. Some studies have shown that an additional 11 days past the day of expected menses are needed to detect $100 \%$ of pregnancies using qualitative tests (29). In addition, pregnancy tests cannot detect a pregnancy resulting from recent sexual intercourse. Qualitative tests also might have positive results for several weeks after termination of pregnancy because hCG can be present for several weeks after delivery or abortion (spontaneous or induced) (33-35).

For contraceptive methods other than IUDs, the benefits of starting to use a contraceptive method likely exceed any risk, even in situations in which the health care provider is uncertain whether the woman is pregnant. Therefore, the health care provider can consider having patients start using contraceptive methods other than IUDs at any time, with a follow-up pregnancy test in 2-4 weeks. The risks of not starting to use contraception should be weighed against the risks of initiating contraception use in a woman who might be already pregnant. Most studies have shown no increased risk for adverse outcomes, including congenital anomalies or neonatal or infant death, among infants exposed in utero to COCs (36-38). Studies also have shown no increased risk for neonatal or infant death or developmental abnormalities among infants exposed in utero to DMPA $(37,39,40)$.

In contrast, for women who want to begin using an IUD (Cu-IUD or LNG-IUD), in situations in which the health care provider is uncertain whether the woman is pregnant, the woman should be provided with another contraceptive method to use until the health care provider is reasonably certain that she is not pregnant and can insert the IUD. Pregnancies among women with IUDs are at higher risk for complications such as spontaneous abortion, septic abortion, preterm delivery, and chorioamnionitis (41).

A systematic review identified four analyses of data from three diagnostic accuracy studies that evaluated the performance of the listed criteria (Box 2) through use of a pregnancy checklist compared with a urine pregnancy test conducted concurrently (42). The performance of the checklist to diagnose or exclude pregnancy varied, with sensitivity of $55 \%-100 \%$ and specificity of $39 \%-89 \%$. The negative predictive value was consistent across studies at 99\%-100\%; the pregnancy checklist correctly ruled out women who were not pregnant. One of the studies assessed the added usefulness of signs and symptoms of pregnancy and found that these criteria did not substantially improve the performance of the pregnancy checklist, although the number of women with signs and symptoms was small (16) (Level of evidence: Diagnostic accuracy studies, fair, direct).

\section{Intrauterine Contraception}

Four IUDs are available in the United States, the coppercontaining IUD and three levonorgestrel-releasing IUDs (containing a total of either $13.5 \mathrm{mg}$ or $52 \mathrm{mg}$ levonorgestrel). Fewer than 1 woman out of 100 becomes pregnant in the first year of using IUDs (with typical use) (14). IUDs are long-acting, are reversible, and can be used by women of all ages, including adolescents, and by parous and nulliparous women. IUDs do not protect against STDs; consistent and correct use of male latex condoms reduces the risk for STDs, including HIV. 


\section{Initiation of Cu-IUDs}

\section{Timing}

- The Cu-IUD can be inserted at any time if it is reasonably certain that the woman is not pregnant (Box 2).

- The $\mathrm{Cu}-\mathrm{IUD}$ also can be inserted within 5 days of the first act of unprotected sexual intercourse as an emergency contraceptive. If the day of ovulation can be estimated, the $\mathrm{Cu}-\mathrm{IUD}$ also can be inserted $>5$ days after sexual intercourse as long as insertion does not occur $>5$ days after ovulation.

\section{Need for Back-Up Contraception}

- No additional contraceptive protection is needed after $\mathrm{Cu}-\mathrm{IUD}$ insertion.

\section{Special Considerations}

\section{Amenorrhea (Not Postpartum)}

- Timing: The Cu-IUD can be inserted at any time if it is reasonably certain that the woman is not pregnant (Box 2).

- Need for back-up contraception: No additional contraceptive protection is needed.

\section{Postpartum (Including After Cesarean Delivery)}

- Timing: The Cu-IUD can be inserted at any time postpartum, including immediately postpartum (U.S. MEC 1 or 2) (Box 1), if it is reasonably certain that the woman is not pregnant (Box 2). The Cu-IUD should not be inserted in a woman with postpartum sepsis (e.g., chorioamnionitis or endometritis) (U.S. MEC 4).

- Need for back-up contraception: No additional contraceptive protection is needed.

\section{Postabortion (Spontaneous or Induced)}

- Timing: The Cu-IUD can be inserted within the first 7 days, including immediately postabortion (U.S. MEC 1 for first-trimester abortion and U.S. MEC 2 for secondtrimester abortion). The $\mathrm{Cu}-\mathrm{IUD}$ should not be inserted immediately after a septic abortion (U.S. MEC 4).

- Need for back-up contraception: No additional contraceptive protection is needed.

\section{Switching from Another Contraceptive Method}

- Timing: The Cu-IUD can be inserted immediately if it is reasonably certain that the woman is not pregnant (Box 2). Waiting for her next menstrual cycle is unnecessary.

- Need for back-up contraception: No additional contraceptive protection is needed.
Comments and Evidence Summary. In situations in which the health care provider is not reasonably certain that the woman is not pregnant, the woman should be provided with another contraceptive method to use until the health care provider can be reasonably certain that she is not pregnant and can insert the $\mathrm{Cu}-\mathrm{IUD}$.

A systematic review identified eight studies that suggested that timing of $\mathrm{Cu}-\mathrm{IUD}$ insertion in relation to the menstrual cycle in non-postpartum women had little effect on longterm outcomes (rates of continuation, removal, expulsion, or pregnancy) or on short-term outcomes (pain at insertion, bleeding at insertion, or immediate expulsion) (43) (Level of evidence: II-2, fair, direct).

\section{Initiation of LNG-IUDs}

\section{Timing of LNG-IUD Insertion}

- The LNG-IUD can be inserted at any time if it is reasonably certain that the woman is not pregnant (Box 2).

\section{Need for Back-Up Contraception}

- If the LNG-IUD is inserted within the first 7 days since menstrual bleeding started, no additional contraceptive protection is needed.

- If the LNG-IUD is inserted $>7$ days since menstrual bleeding started, the woman needs to abstain from sexual intercourse or use additional contraceptive protection for the next 7 days.

\section{Special Considerations}

\section{Amenorrhea (Not Postpartum)}

- Timing: The LNG-IUD can be inserted at any time if it is reasonably certain that the woman is not pregnant (Box 2).

- Need for back-up contraception: The woman needs to abstain from sexual intercourse or use additional contraceptive protection for the next 7 days.

\section{Postpartum (Including After Cesarean Delivery)}

- Timing: The LNG-IUD can be inserted at any time, including immediately postpartum (U.S. MEC 1 or 2 ) if it is reasonably certain that the woman is not pregnant (Box 2). The LNG-IUD should not be inserted in a woman with postpartum sepsis (e.g., chorioamnionitis or endometritis) (U.S. MEC 4).

- Need for back-up contraception: If the woman is $<6$ months postpartum, amenorrheic, and fully or nearly fully breastfeeding (exclusively breastfeeding or the vast majority $[\geq 85 \%]$ of feeds are breastfeeds) (27), no 
additional contraceptive protection is needed. Otherwise, a woman who is $\geq 21$ days postpartum and has not experienced return of her menstrual cycle needs to abstain from sexual intercourse or use additional contraceptive protection for the next 7 days. If her menstrual cycles have returned and it has been $>7$ days since menstrual bleeding began, she needs to abstain from sexual intercourse or use additional contraceptive protection for the next 7 days.

\section{Postabortion (Spontaneous or Induced)}

- Timing: The LNG-IUD can be inserted within the first 7 days, including immediately postabortion (U.S. MEC 1 for first-trimester abortion and U.S. MEC 2 for secondtrimester abortion). The LNG-IUD should not be inserted immediately after a septic abortion (U.S. MEC 4).

- Need for back-up contraception: The woman needs to abstain from sexual intercourse or use additional contraceptive protection for the next 7 days unless the IUD is placed at the time of a surgical abortion.

\section{Switching from Another Contraceptive Method}

- Timing: The LNG-IUD can be inserted immediately if it is reasonably certain that the woman is not pregnant (Box 2). Waiting for her next menstrual cycle is unnecessary.

- Need for back-up contraception: If it has been $>7$ days since menstrual bleeding began, the woman needs to abstain from sexual intercourse or use additional contraceptive protection for the next 7 days.

- Switching from a Cu-IUD: If the woman has had sexual intercourse since the start of her current menstrual cycle and it has been $>5$ days since menstrual bleeding started, theoretically, residual sperm might be in the genital tract, which could lead to fertilization if ovulation occurs. A health care provider may consider providing any type of ECPs at the time of LNG-IUD insertion.

Comments and Evidence Summary. In situations in which the health care provider is uncertain whether the woman might be pregnant, the woman should be provided with another contraceptive method to use until the health care provider can be reasonably certain that she is not pregnant and can insert the LNG-IUD. If a woman needs to use additional contraceptive protection when switching to an LNG-IUD from another contraceptive method, consider continuing her previous method for 7 days after LNG-IUD insertion. No direct evidence was found regarding the effects of inserting LNG-IUDs on different days of the cycle on short- or longterm outcomes (43).

\section{Examinations and Tests Needed Before Initiation of a Cu-IUD or an LNG-IUD}

Among healthy women, few examinations or tests are needed before initiation of an IUD (Table 1). Bimanual examination and cervical inspection are necessary before IUD insertion. A baseline weight and BMI measurement might be useful for monitoring IUD users over time. If a woman has not been screened for STDs according to STD screening guidelines, screening can be performed at the time of insertion. Women with known medical problems or other special conditions might need additional examinations or tests before being determined to be appropriate candidates for a particular method of contraception. U.S. MEC might be useful in such circumstances (5).

Comments and Evidence Summary. Weight (BMI): Obese women can use IUDs (U.S. MEC 1) (5); therefore, screening for obesity is not necessary for the safe initiation of IUDs. However, measuring weight and calculating

TABLE 1. Classification of examinations and tests needed before IUD insertion

\begin{tabular}{|c|c|c|}
\hline \multirow[b]{2}{*}{ Examination or test } & \multicolumn{2}{|c|}{ Class* } \\
\hline & $\begin{array}{c}\text { Copper- } \\
\text { containing IUD }\end{array}$ & $\begin{array}{l}\text { Levonorgestrel- } \\
\text { releasing IUD }\end{array}$ \\
\hline \multicolumn{3}{|l|}{ Examination } \\
\hline Blood pressure & $\mathrm{C}$ & $\mathrm{C}$ \\
\hline Weight (BMI) (weight $[\mathrm{kg}] /$ height $[\mathrm{m}]^{2}$ ) & $-^{\dagger}$ & $-^{\dagger}$ \\
\hline Clinical breast examination & $\mathrm{C}$ & $\mathrm{C}$ \\
\hline Bimanual examination and cervical inspection & A & A \\
\hline \multicolumn{3}{|l|}{ Laboratory test } \\
\hline Glucose & C & C \\
\hline Lipids & C & C \\
\hline Liver enzymes & C & C \\
\hline Hemoglobin & C & C \\
\hline Thrombogenic mutations & C & C \\
\hline Cervical cytology (Papanicolaou smear) & C & C \\
\hline STD screening with laboratory tests & —§ & —§ \\
\hline HIV screening with laboratory tests & C & C \\
\hline
\end{tabular}

Abbreviations: $\mathrm{BMI}=$ body mass index; $\mathrm{HIV}=$ human immunodeficiency virus; IUD = intrauterine device; STD = sexually transmitted disease; U.S. MEC = U.S. Medical Eligibility Criteria for Contraceptive Use.

* Class A: essential and mandatory in all circumstances for safe and effective use of the contraceptive method. Class B: contributes substantially to safe and effective use, but implementation may be considered within the public health and/or service context; the risk of not performing an examination or test should be balanced against the benefits of making the contraceptive method available. Class C: does not contribute substantially to safe and effective use of the contraceptive method.

† Weight (BMI) measurement is not needed to determine medical eligibility for any methods of contraception because all methods can be used (U.S. MEC 1) or generally can be used (U.S. MEC 2) among obese women (Box 1). However, measuring weight and calculating BMl at baseline might be helpful for monitoring any changes and counseling women who might be concerned about weight change perceived to be associated with their contraceptive method.

$\S$ Most women do not require additional STD screening at the time of IUD insertion. If a woman with risk factors for STDs has not been screened for gonorrhea and chlamydia according to CDC's STD Treatment Guidelines (http://www.cdc.gov/std/ treatment), screening can be performed at the time of IUD insertion, and insertion should not be delayed. Women with current purulent cervicitis or chlamydial infection or gonococcal infection should not undergo IUD insertion (U.S. MEC 4). 
BMI (weight $[\mathrm{kg}] /$ height $\left[\mathrm{m}^{2}\right]$ ) at baseline might be helpful for monitoring any changes and counseling women who might be concerned about weight change perceived to be associated with their contraceptive method.

Bimanual examination and cervical inspection: Bimanual examination and cervical inspection are necessary before IUD insertion to assess uterine size and position and to detect any cervical or uterine abnormalities that might indicate infection or otherwise prevent IUD insertion $(44,45)$.

STDs: Women should be routinely screened for chlamydial infection and gonorrhea according to national screening guidelines. The CDC Sexually Transmitted Diseases Treatment Guidelines provide information on screening eligibility, timing, and frequency of screening and on screening for persons with risk factors (15) (http://www.cdc.gov/std/treatment). If STD screening guidelines have been followed, most women do not need additional STD screening at the time of IUD insertion, and insertion should not be delayed. If a woman with risk factors for STDs has not been screened for gonorrhea and chlamydia according to CDC STD treatment guidelines, screening can be performed at the time of IUD insertion, and insertion should not be delayed. Women with current purulent cervicitis or chlamydial infection or gonococcal infection should not undergo IUD insertion (U.S. MEC 4). A systematic review identified two studies that demonstrated no differences in PID rates among women who screened positive for gonorrhea or chlamydia and underwent concurrent IUD insertion compared with women who screened positive and initiated other contraceptive methods (46). Indirect evidence demonstrates women who undergo same-day STD screening and IUD insertion have similar PID rates compared with women who have delayed IUD insertion. Women who undergo same-day STD screening and IUD insertion have low incidence rates of PID. Algorithms for predicting PID among women with risk factors for STDs have poor predictive value. Risk for PID among women with risk factors for STDs is low (15,47-57). Although women with STDs at the time of IUD insertion have a higher risk for PID, the overall rate of PID among all IUD users is low $(51,54)$.

Hemoglobin: Women with iron-deficiency anemia can use the LNG-IUD (U.S. MEC 1) (5); therefore, screening for anemia is not necessary for safe initiation of the LNGIUD. Women with iron-deficiency anemia generally can use $\mathrm{Cu}-\mathrm{IUDs}$ (U.S. MEC 2) (5). Measurement of hemoglobin before initiation of $\mathrm{Cu}-\mathrm{IUDs}$ is not necessary because of the minimal change in hemoglobin among women with and without anemia using Cu-IUDs. A systematic review identified four studies that provided direct evidence for changes in hemoglobin among women with anemia who received $\mathrm{Cu}$-IUDs (58). Evidence from one randomized trial (59) and one prospective cohort study $(60)$ showed no significant changes in hemoglobin among $\mathrm{Cu}$-IUD users with anemia, whereas two prospective cohort studies $(61,62)$ showed a statistically significant decrease in hemoglobin levels during 12 months of follow-up; however, the magnitude of the decrease was small and most likely not clinically significant. The systematic review also identified 21 studies that provided indirect evidence by examining changes in hemoglobin among healthy women receiving $\mathrm{Cu}-\mathrm{IUDs}$ (63-83), which generally showed no clinically significant changes in hemoglobin levels with up to 5 years of follow up (Level of evidence: I to II-2, fair, direct).

Lipids: Screening for dyslipidemias is not necessary for the safe initiation of $\mathrm{Cu}$-IUD or LNG-IUD because of the low prevalence of undiagnosed disease in women of reproductive age and the low likelihood of clinically significant changes with use of hormonal contraceptives. A systematic review did not identify any evidence regarding outcomes among women who were screened versus not screened with lipid measurement before initiation of hormonal contraceptives (57). During 2009-2012 among women aged 20-44 years in the United States, 7.6\% had high cholesterol, defined as total serum cholesterol $\geq 240 \mathrm{mg} / \mathrm{dL}$ (84). During 1999-2008, the prevalence of undiagnosed hypercholesterolemia among women aged $20-44$ years was approximately $2 \%$ (85). Studies have shown mixed results about the effects of hormonal methods on lipid levels among both healthy women and women with baseline lipid abnormalities, and the clinical significance of these changes is unclear $(86-89)$.

Liver enzymes: Women with liver disease can use the Cu-IUD (U.S. MEC 1) (5); therefore, screening for liver disease is not necessary for the safe initiation of the Cu-IUD. Although women with certain liver diseases generally should not use the LNG-IUD (U.S. MEC 3) (5), screening for liver disease before initiation of the LNG-IUD is not necessary because of the low prevalence of these conditions and the high likelihood that women with liver disease already would have had the condition diagnosed. A systematic review did not identify any evidence regarding outcomes among women who were screened versus not screened with liver enzyme tests before initiation of hormonal contraceptive use (57). In 2012, among U.S. women, the percentage with liver disease (not further specified) was $1.3 \%$ (90). In 2013, the incidence of acute hepatitis A, B, or $\mathrm{C}$ was $\leq 1$ per 100,000 U.S. population (91). During 2002-2011, the incidence of liver carcinoma among U.S. women was approximately 3.7 per 100,000 population (92). Because estrogen and progestins are metabolized in the liver, the use of hormonal contraceptives among women with liver disease might, theoretically, be a concern. The use of hormonal contraceptives, specifically COCs and POPs, 
does not affect disease progression or severity in women with hepatitis, cirrhosis, or benign focal nodular hyperplasia $(93,94)$, although evidence is limited, and no evidence exists for the LNG-IUD.

Clinical breast examination: Women with breast disease can use the Cu-IUD (U.S. MEC 1) (5); therefore, screening for breast disease is not necessary for the safe initiation of the Cu-IUD. Although women with current breast cancer should not use the LNG-IUD (U.S. MEC 4) (5), screening asymptomatic women with a clinical breast examination before inserting an IUD is not necessary because of the low prevalence of breast cancer among women of reproductive age. A systematic review did not identify any evidence regarding outcomes among women who were screened versus not screened with a breast examination before initiation of hormonal contraceptives (95). The incidence of breast cancer among women of reproductive age in the United States is low. In 2012, the incidence of breast cancer among women aged 20-49 years was approximately 70.7 per 100,000 women (96).

Cervical cytology: Although women with cervical cancer should not undergo IUD insertion (U.S. MEC 4) (5), screening asymptomatic women with cervical cytology before IUD insertion is not necessary because of the high rates of cervical screening, low incidence of cervical cancer in the United States, and high likelihood that a woman with cervical cancer already would have had the condition diagnosed. A systematic review did not identify any evidence regarding outcomes among women who were screened versus not screened with cervical cytology before initiation of IUDs (57). Cervical cancer is rare in the United States, with an incidence rate of 9.8 per 100,000 women during 2012 (96). The incidence and mortality rates from cervical cancer have declined dramatically in the United States, largely because of cervical cytology screening (97). Overall screening rates for cervical cancer in the United States are high; in 2013 among women aged $18-44$ years, approximately $77 \%$ reported having cervical cytology screening within the last 3 years (98).

HIV screening: Women with HIV infection can use (U.S. MEC 1) or generally can use (U.S. MEC 2) IUDs (5). Therefore, HIV screening is not necessary before IUD insertion. A systematic review did not identify any evidence regarding outcomes among women who were screened versus not screened for HIV infection before IUD insertion (57). Limited evidence suggests that IUDs are not associated with disease progression, increased infection, or other adverse health effects among women with HIV infection (99-114).

Other screening: Women with hypertension, diabetes, or thrombogenic mutations can use (U.S. MEC 1) or generally can use (U.S. MEC 2) IUDs (5). Therefore, screening for these conditions is not necessary for the safe initiation of IUDs.

\section{Provision of Medications to Ease IUD Insertion}

- Misoprostol is not recommended for routine use before IUD insertion. Misoprostol might be helpful in select circumstances (e.g., in women with a recent failed insertion).

- Paracervical block with lidocaine might reduce patient pain during IUD insertion.

Comments and Evidence Summary. Potential barriers to IUD use include anticipated pain with insertion and provider concerns about difficult insertion. Identifying effective approaches to ease IUD insertion might increase IUD initiation.

Evidence for misoprostol from two systematic reviews, including a total of 10 randomized controlled trials, suggests that misoprostol does not improve provider ease of insertion, reduce the need for adjunctive insertion measures, or improve insertion success (Level of evidence: I, good to fair, direct) and might increase patient pain and side effects (Level of evidence: I, high quality) $(115,116)$. However, one randomized controlled trial examined women with a recent failed IUD insertion and found significantly higher insertion success with second insertion attempt among women pretreated with misoprostol versus placebo (Level of evidence: I, good, direct) (117).

Limited evidence for paracervical block with lidocaine from one systematic review suggests that it might reduce patient pain (115). In this review, two randomized controlled trials found significantly reduced pain at either tenaculum placement or IUD insertion among women receiving paracervical block with $1 \%$ lidocaine 3-5 minutes before IUD insertion (118,119). Neither trial found differences in side effects among women receiving paracervical block compared with controls (Level of evidence: I, moderate to low quality) $(118,119)$.

Limited evidence on nonsteroidal antiinflammatory drugs (NSAIDs) and nitric oxide donors generally suggested no positive effect; evidence on lidocaine with administration other than paracervical block was limited and inconclusive (Level of evidence for provider ease of insertion: I, good to poor, direct; Level of evidence for need for adjunctive insertion measures: I, fair, direct; Level of evidence for patient pain: I, high to low quality; Level of evidence for side effects: I, high to low quality) $(115,116)$.

\section{Provision of Prophylactic Antibiotics at the Time of IUD Insertion}

- Prophylactic antibiotics are generally not recommended for Cu-IUD or LNG-IUD insertion.

Comments and Evidence Summary. Theoretically, IUD insertion could induce bacterial spread and lead to 
complications such as PID or infective endocarditis. A metaanalysis was conducted of randomized controlled trials examining antibiotic prophylaxis versus placebo or no treatment for IUD insertion (120). Use of prophylaxis reduced the frequency of unscheduled return visits but did not significantly reduce the incidence of PID or premature IUD discontinuation. Although the risk for PID was higher within the first 20 days after insertion, the incidence of PID was low among all women who had IUDs inserted (51). In addition, the American Heart Association recommends that the use of prophylactic antibiotics solely to prevent infective endocarditis is not needed for genitourinary procedures (121). Studies have not demonstrated a conclusive link between genitourinary procedures and infective endocarditis or a preventive benefit of prophylactic antibiotics during such procedures (121).

\section{Routine Follow-Up After IUD Insertion}

These recommendations address when routine follow-up is needed for safe and effective continued use of contraception for healthy women. The recommendations refer to general situations and might vary for different users and different situations. Specific populations who might benefit from more frequent follow-up visits include adolescents, persons with certain medical conditions or characteristics, and persons with multiple medical conditions.

- Advise the woman to return at any time to discuss side effects or other problems, if she wants to change the method being used, and when it is time to remove or replace the contraceptive method. No routine follow-up visit is required.

- At other routine visits, health care providers who see IUD users should do the following:

- Assess the woman's satisfaction with her contraceptive method and whether she has any concerns about method use.

- Assess any changes in health status, including medications, that would change the appropriateness of the IUD for safe and effective continued use on the basis of U.S. MEC (e.g., category 3 and 4 conditions and characteristics).

- Consider performing an examination to check for the presence of the IUD strings.

- Consider assessing weight changes and counseling women who are concerned about weight changes perceived to be associated with their contraceptive method.

Comments and Evidence Summary. Evidence from a systematic review about the effect of a specific follow-up visit schedule on IUD continuation is very limited and of poor quality. The evidence did not suggest that greater frequency of visits or earlier timing of the first follow-up visit after insertion improves continuation of use (122) (Level of evidence: II-2, poor, direct). Evidence from four studies from a systematic review on the incidence of PID among IUD initiators, or IUD removal as a result of PID, suggested that the incidence of PID did not differ between women using Cu- IUDs and those using DMPA, COCs, or LNG-IUDs (123) (Level of evidence: I to II-2, good, indirect). Evidence on the timing of PID after IUD insertion is mixed. Although the rate of PID generally was low, the largest study suggested that the rate of PID was significantly higher in the first 20 days after insertion (51) (Level of evidence: I to II-3, good to poor, indirect).

\section{Bleeding Irregularities with Cu-IUD Use}

- Before Cu-IUD insertion, provide counseling about potential changes in bleeding patterns during $\mathrm{Cu}-\mathrm{IUD}$ use. Unscheduled spotting or light bleeding, as well as heavy or prolonged bleeding, is common during the first 3-6 months of Cu-IUD use, is generally not harmful, and decreases with continued $\mathrm{Cu}-\mathrm{IUD}$ use.

- If clinically indicated, consider an underlying gynecological problem, such as Cu-IUD displacement, an STD, pregnancy, or new pathologic uterine conditions (e.g., polyps or fibroids), especially in women who have already been using the $\mathrm{Cu}$-IUD for a few months or longer and who have developed a new onset of heavy or prolonged bleeding. If an underlying gynecological problem is found, treat the condition or refer for care.

- If an underlying gynecological problem is not found and the woman requests treatment, the following treatment option can be considered during days of bleeding:

- NSAIDs for short-term treatment (5-7 days)

- If bleeding persists and the woman finds it unacceptable, counsel her on alternative contraceptive methods, and offer another method if it is desired.

Comments and Evidence Summary. During contraceptive counseling and before insertion of the $\mathrm{Cu}-\mathrm{IUD}$, information about common side effects such as unscheduled spotting or light bleeding or heavy or prolonged menstrual bleeding, especially during the first 3-6 months of use, should be discussed (64). These bleeding irregularities are generally not harmful. Enhanced counseling about expected bleeding patterns and reassurance that bleeding irregularities are generally not harmful has been shown to reduce method discontinuation in clinical trials with other contraceptives (i.e., DMPA) $(124,125)$.

Evidence is limited on specific drugs, doses, and durations of use for effective treatments for bleeding irregularities with $\mathrm{Cu}-\mathrm{IUD}$ use. Therefore, although this report includes general 
recommendations for treatments to consider, evidence for specific regimens is lacking.

A systematic review identified 11 studies that examined various therapeutic treatments for heavy menstrual bleeding, prolonged menstrual bleeding, or both among women using $\mathrm{Cu}$-IUDs (126). Nine studies examined the use of various oral NSAIDs for the treatment of heavy or prolonged menstrual bleeding among $\mathrm{Cu}-\mathrm{IUD}$ users and compared them with either a placebo or a baseline cycle. Three of these trials examined the use of indomethacin (127-129), three examined mefenamic acid (130-132), and three examined flufenamic acid $(127,128,133)$. Other NSAIDs used in the reported trials included alclofenac $(127,128)$, suprofen $(134)$, and diclofenac sodium (135). All but one NSAID study (131) demonstrated statistically significant or notable reductions in mean total menstrual blood loss with NSAID use. One study among $19 \mathrm{Cu}$-IUD users with heavy bleeding suggested that treatment with oral tranexamic acid can significantly reduce mean blood loss during treatment compared with placebo (135). Data regarding the overall safety of tranexamic acid are limited; an FDA warning states that tranexamic acid is contraindicated in women with active thromboembolic disease or with a history or intrinsic risk for thrombosis or thromboembolism $(136,137)$. Treatment with aspirin demonstrated no statistically significant change in mean blood loss among women whose pretreatment menstrual blood loss was $>80 \mathrm{ml}$ or $60-80 \mathrm{~mL}$; treatment resulted in a significant increase among women whose pretreatment menstrual blood loss was $<60 \mathrm{~mL}$ (138). One study examined the use of a synthetic form of vasopressin, intranasal desmopressin $(300 \mu \mathrm{g} /$ day $)$, for the first 5 days of menses for three treatment cycles and found a significant reduction in mean blood loss compared with baseline (130) (Level of evidence: I to II-3, poor to fair, direct). Only one small study examined treatment of spotting with three separate NSAIDs and did not observe improvements in spotting in any of the groups (127) (Level of evidence: I, poor, direct).

\section{Bleeding Irregularities (Including Amenorrhea) with LNG-IUD Use}

- Before LNG-IUD insertion, provide counseling about potential changes in bleeding patterns during LNG-IUD use. Unscheduled spotting or light bleeding is expected during the first 3-6 months of LNG-IUD use, is generally not harmful, and decreases with continued LNG-IUD use. Over time, bleeding generally decreases with LNGIUD use, and many women experience only light menstrual bleeding or amenorrhea. Heavy or prolonged bleeding, either unscheduled or menstrual, is uncommon during LNG-IUD use.

\section{Irregular Bleeding (Spotting, Light Bleeding, or Heavy or Prolonged Bleeding)}

- If clinically indicated, consider an underlying gynecological problem, such as LNG-IUD displacement, an STD, pregnancy, or new pathologic uterine conditions (e.g., polyps or fibroids). If an underlying gynecological problem is found, treat the condition or refer for care.

- If bleeding persists and the woman finds it unacceptable, counsel her on alternative contraceptive methods, and offer another method if it is desired.

\section{Amenorrhea}

- Amenorrhea does not require any medical treatment. Provide reassurance.

- If a woman's regular bleeding pattern changes abruptly to amenorrhea, consider ruling out pregnancy if clinically indicated.

- If amenorrhea persists and the woman finds it unacceptable, counsel her on alternative contraceptive methods, and offer another method if it is desired

Comments and Evidence Summary. During contraceptive counseling and before insertion of the LNG-IUD, information about common side effects such as unscheduled spotting or light bleeding, especially during the first 3-6 months of use, should be discussed. Approximately half of LNG-IUD users are likely to experience amenorrhea or oligomenorrhea by 2 years of use (139). These bleeding irregularities are generally not harmful. Enhanced counseling about expected bleeding patterns and reassurance that bleeding irregularities are generally not harmful has been shown to reduce method discontinuation in clinical trials with other hormonal contraceptives (i.e., DMPA) $(124,125)$. No direct evidence was found regarding therapeutic treatments for bleeding irregularities during LNG-IUD use.

\section{Management of the IUD when a Cu-IUD or an LNG-IUD User Is Found To Have PID}

- Treat the PID according to the CDC Sexually Transmitted Diseases Treatment Guidelines (15).

- Provide comprehensive management for STDs, including counseling about condom use.

- The IUD does not need to be removed immediately if the woman needs ongoing contraception.

- Reassess the woman in 48-72 hours. If no clinical improvement occurs, continue antibiotics and consider removal of the IUD.

- If the woman wants to discontinue use, remove the IUD sometime after antibiotics have been started to avoid the 
potential risk for bacterial spread resulting from the removal procedure.

- If the IUD is removed, consider ECPs if appropriate. Counsel the woman on alternative contraceptive methods, and offer another method if it is desired.

- A summary of IUD management in women with PID is provided (Appendix F).

Comments and Evidence Summary. Treatment outcomes do not generally differ between women with PID who retain the IUD and those who have the IUD removed; however, appropriate antibiotic treatment and close clinical follow-up are necessary.

A systematic review identified four studies that included women using copper or nonhormonal IUDs who developed PID and compared outcomes between women who had the IUD removed or did not (140). One randomized trial showed that women with IUDs removed had longer hospitalizations than those who did not, although no differences in PID recurrences or subsequent pregnancies were observed (141). Another randomized trial showed no differences in laboratory findings among women who removed the IUD compared with those who did not (142). One prospective cohort study showed no differences in clinical or laboratory findings during hospitalization; however, the IUD removal group had longer hospitalizations (143). One randomized trial showed that the rate of recovery for most clinical signs and symptoms was higher among women who had the IUD removed than among women who did not (144). No evidence was found regarding women using LNG-IUDs (Level of evidence: I to II-2, fair, direct.)

\section{Management of the IUD when a Cu-IUD or an LNG-IUD User is Found To Be Pregnant}

- Evaluate for possible ectopic pregnancy.

- Advise the woman that she has an increased risk for spontaneous abortion (including septic abortion that might be life threatening) and for preterm delivery if the IUD is left in place. The removal of the IUD reduces these risks but might not decrease the risk to the baseline level of a pregnancy without an IUD.

- If she does not want to continue the pregnancy, counsel her about options.

- If she wants to continue the pregnancy, advise her to seek care promptly if she has heavy bleeding, cramping, pain, abnormal vaginal discharge, or fever.

\section{IUD Strings Are Visible or Can Be Retrieved Safely from the Cervical Canal}

- Advise the woman that the IUD should be removed as soon as possible.
- If the IUD is to be removed, remove it by pulling on the strings gently.

- Advise the woman that she should return promptly if she has heavy bleeding, cramping, pain, abnormal vaginal discharge, or fever.

- If she chooses to keep the IUD, advise her to seek care promptly if she has heavy bleeding, cramping, pain, abnormal vaginal discharge, or fever.

\section{IUD Strings Are Not Visible and Cannot Be Safely Retrieved}

- If ultrasonography is available, consider performing or referring for ultrasound examination to determine the location of the IUD. If the IUD cannot be located, it might have been expelled or have perforated the uterine wall.

- If ultrasonography is not possible or the IUD is determined by ultrasound to be inside the uterus, advise the woman to seek care promptly if she has heavy bleeding, cramping, pain, abnormal vaginal discharge, or fever.

Comments and Evidence Summary. Removing the IUD improves the pregnancy outcome if the IUD strings are visible or the device can be retrieved safely from the cervical canal. Risks for spontaneous abortion, preterm delivery, and infection are substantial if the IUD is left in place.

Theoretically, the fetus might be affected by hormonal exposure from an LNG-IUD. However, whether this exposure increases the risk for fetal abnormalities is unknown.

A systematic review identified nine studies suggesting that women who did not remove their IUDs during pregnancy were at greater risk for adverse pregnancy outcomes (including spontaneous abortion, septic abortion, preterm delivery, and chorioamnionitis) compared with women who had their IUDs removed or who did not have an IUD (41). Cu-IUD removal decreased risks but not to the baseline risk for pregnancies without an IUD. One case series examined LNG-IUDs. When they were not removed, 8 out of 10 pregnancies ended in spontaneous abortions (Level of evidence: II-2, fair, direct).

\section{Implants}

The etonogestrel implant, a single rod with $68 \mathrm{mg}$ of etonogestrel, is available in the United States. Fewer than 1 woman out of 100 become pregnant in the first year of use of the etonogestrel implant with typical use (14). The implant is long acting, is reversible, and can be used by women of all ages, including adolescents. The implant does not protect against STDs; consistent and correct use of male latex condoms reduces the risk for STDs, including HIV. 


\section{Initiation of Implants}

\section{Timing}

- The implant can be inserted at any time if it is reasonably certain that the woman is not pregnant (Box 2).

\section{Need for Back-Up Contraception}

- If the implant is inserted within the first 5 days since menstrual bleeding started, no additional contraceptive protection is needed.

- If the implant is inserted $>5$ days since menstrual bleeding started, the woman needs to abstain from sexual intercourse or use additional contraceptive protection for the next 7 days.

\section{Special Considerations}

\section{Amenorrhea (Not Postpartum)}

- Timing: The implant can be inserted at any time if it is reasonably certain that the woman is not pregnant (Box 2).

- Need for back-up contraception: The woman needs to abstain from sexual intercourse or use additional contraceptive protection for the next 7 days.

\section{Postpartum (Breastfeeding)}

- Timing: The implant can be inserted at any time (U.S. MEC 2 if $<1$ month postpartum and U.S. MEC 1 if $\geq 1$ month postpartum) if it is reasonably certain that the woman is not pregnant (Box 2).

- Need for back-up contraception: If the woman is $<6$ months postpartum, amenorrheic, and fully or nearly fully breastfeeding (exclusively breastfeeding or the vast majority $[\geq 85 \%]$ of feeds are breastfeeds) (27), no additional contraceptive protection is needed. Otherwise, a woman who is $\geq 21$ days postpartum and has not experienced return of her menstrual cycle needs to abstain from sexual intercourse or use additional contraceptive protection for the next 7 days. If her menstrual cycles have returned and it has been $>5$ days since menstrual bleeding started, she needs to abstain from sexual intercourse or use additional contraceptive protection for the next 7 days.

\section{Postpartum (Not Breastfeeding)}

- Timing: The implant can be inserted at any time, including immediately postpartum (U.S. MEC 1) if it is reasonably certain that the woman is not pregnant (Box 2).

- Need for back-up contraception: If a woman is $<21$ days postpartum, no additional contraceptive protection is needed. A woman who is $\geq 21$ days postpartum and has not experienced return of her menstrual cycle needs to abstain from sexual intercourse or use additional contraceptive protection for the next 7 days. If her menstrual cycles have returned and it has been $>5$ days since menstrual bleeding started, she needs to abstain from sexual intercourse or use additional contraceptive protection for the next 7 days.

\section{Postabortion (Spontaneous or Induced)}

- Timing: The implant can be inserted within the first 7 days, including immediately after the abortion (U.S. MEC 1).

- Need for back-up contraception: The woman needs to abstain from sexual intercourse or use additional contraceptive protection for the next 7 days unless the implant is placed at the time of a surgical abortion.

\section{Switching from Another Contraceptive Method}

- Timing: The implant can be inserted immediately if it is reasonably certain that the woman is not pregnant (Box 2). Waiting for her next menstrual cycle is unnecessary.

- Need for back-up contraception: If it has been $>5$ days since menstrual bleeding started, the woman needs to abstain from sexual intercourse or use additional contraceptive protection for the next 7 days after insertion.

- Switching from an IUD: If the woman has had sexual intercourse since the start of her current menstrual cycle and it has been $>5$ days since menstrual bleeding started, theoretically, residual sperm might be in the genital tract, which could lead to fertilization if ovulation occurs. A health care provider may consider any of the following options:

- Advise the woman to retain the IUD for at least 7 days after the implant is inserted and return for IUD removal.

- Advise the woman to abstain from sexual intercourse or use barrier contraception for 7 days before removing the IUD and switching to the new method.

- If the woman cannot return for IUD removal and has not abstained from sexual intercourse or used barrier contraception for 7 days, advise the woman to use ECPs (with the exception of UPA) at the time of IUD removal.

Comments and Evidence Summary. In situations in which the health care provider is uncertain whether the woman might be pregnant, the benefits of starting the implant likely exceed any risk; therefore, starting the implant should be considered at any time, with a follow-up pregnancy test in 2-4 weeks.

If a woman needs to use additional contraceptive protection when switching to an implant from another contraceptive method, consider continuing her previous method for 7 days after implant insertion. No direct evidence was found regarding the effects of starting the etonogestrel implant at different times of the cycle. 


\section{Examinations and Tests Needed Before Implant Insertion}

Among healthy women, no examinations or tests are needed before initiation of an implant, although a baseline weight and BMI measurement might be useful for monitoring implant users over time (Table 2). Women with known medical problems or other special conditions might need additional examinations or tests before being determined to be appropriate candidates for a particular method of contraception. U.S. MEC might be useful in such circumstances (5).

Comments and Evidence Summary. Weight (BMI): Obese women can use implants (U.S. MEC 1) (5); therefore, screening for obesity is not necessary for the safe initiation of implants. However, measuring weight and calculating BMI at baseline might be helpful for monitoring any changes and counseling women who might be concerned about weight change perceived to be associated with their contraceptive method.

Bimanual examination and cervical inspection: A pelvic examination is not necessary before initiation of implants because it would not facilitate detection of conditions for which implant use would be unsafe. Women with current breast cancer should not use implants (U.S. MEC 4); women with certain liver diseases generally should not (U.S. MEC 3) use implants (5). However, none of these conditions are likely to be detected

TABLE 2. Classification of examinations and tests needed before implant insertion

\begin{tabular}{ll}
\hline Examination or test & Class* $^{*}$ \\
\hline Examination & \\
Blood pressure & $\mathrm{C}$ \\
Weight $(\mathrm{BMI})\left(\right.$ weight $[\mathrm{kg}] /$ height $[\mathrm{m}]^{2}$ ) & $\mathrm{C}^{+}$ \\
Clinical breast examination & $\mathrm{C}$ \\
Bimanual examination and cervical inspection & \\
Laboratory test & $\mathrm{C}$ \\
Glucose & $\mathrm{C}$ \\
Lipids & $\mathrm{C}$ \\
Liver enzymes & $\mathrm{C}$ \\
Hemoglobin & $\mathrm{C}$ \\
Thrombogenic mutations & $\mathrm{C}$ \\
Cervical cytology (Papanicolaou smear) & $\mathrm{C}$ \\
STD screening with laboratory tests & $\mathrm{C}$ \\
HIV screening with laboratory tests &
\end{tabular}

Abbreviations: $\mathrm{BMI}=$ body mass index; $\mathrm{HIV}=$ human immunodeficiency virus; $\mathrm{STD}=$ sexually transmitted disease; U.S. MEC = U.S. Medical Eligibility Criteria for Contraceptive Use.

* Class A: essential and mandatory in all circumstances for safe and effective use of the contraceptive method. Class B: contributes substantially to safe and effective use, but implementation may be considered within the public health and/or service context; the risk of not performing an examination or test should be balanced against the benefits of making the contraceptive method available. Class C: does not contribute substantially to safe and effective use of the contraceptive method.

+ Weight (BMI) measurement is not needed to determine medical eligibility for any methods of contraception because all methods can be used (U.S. MEC 1) or generally can be used (U.S. MEC 2) among obese women (Box 1). However, measuring weight and calculating BMl at baseline might be helpful for monitoring any changes and counseling women who might be concerned about weight change perceived to be associated with their contraceptive method. by pelvic examination (145). A systematic review identified two case-control studies that compared delayed and immediate pelvic examination before initiation of hormonal contraceptives, specifically oral contraceptives or DMPA (95). No differences in risk factors for cervical neoplasia, incidence of STDs, incidence of abnormal Papanicolaou smears, or incidence of abnormal wet mounts were observed. No evidence was found regarding implants (Level of evidence: II-2 fair, direct).

Lipids: Screening for dyslipidemias is not necessary for the safe initiation of implants because of the low prevalence of undiagnosed disease in women of reproductive age and the low likelihood of clinically significant changes with use of hormonal contraceptives. A systematic review did not identify any evidence regarding outcomes among women who were screened versus not screened with lipid measurement before initiation of hormonal contraceptives (57). During 2009-2012 among women aged 20-44 years in the United States, $7.6 \%$ had high cholesterol, defined as total serum cholesterol $\geq 240 \mathrm{mg} /$ dL (84). During 1999-2008, the prevalence of undiagnosed hypercholesterolemia among women aged 20-44 years was approximately $2 \%$ (85). Studies have shown mixed results regarding the effects of hormonal methods on lipid levels among both healthy women and women with baseline lipid abnormalities, and the clinical significance of these changes is unclear (86-89).

Liver enzymes: Although women with certain liver diseases generally should not use implants (U.S. MEC 3) (5), screening for liver disease before initiation of implants is not necessary because of the low prevalence of these conditions and the high likelihood that women with liver disease already would have had the condition diagnosed. A systematic review did not identify any evidence regarding outcomes among women who were screened versus not screened with liver enzyme tests before initiation of hormonal contraceptives (57). In 2012, the percentage of U.S. women with liver disease (not further specified) was $1.3 \%(90)$. In 2013, the incidence of acute hepatitis A, B, or $\mathrm{C}$ was $\leq 1$ per 100,000 U.S. population (91). During 2002-2011, the incidence of liver carcinoma among U.S. women was approximately 3.7 per 100,000 population (92). Because estrogen and progestins are metabolized in the liver, the use of hormonal contraceptives among women with liver disease might, theoretically, be a concern. The use of hormonal contraceptives, specifically COCs and POPs, does not affect disease progression or severity in women with hepatitis, cirrhosis, or benign focal nodular hyperplasia $(93,94)$, although evidence is limited and no evidence exists for implants.

Clinical breast examination: Although women with current breast cancer should not use implants (U.S. MEC 4) (5), screening asymptomatic women with a clinical breast 
examination before initiation of implants is not necessary because of the low prevalence of breast cancer among women of reproductive age (15-49 years). A systematic review did not identify any evidence regarding outcomes among women who were screened versus not screened with a breast examination before initiation of hormonal contraceptives (95). The incidence of breast cancer among women of reproductive age in the United States is low. In 2012, the incidence of breast cancer among women aged 20-49 years was approximately 70.7 per 100,000 women (96).

Other screening: Women with hypertension, diabetes, anemia, thrombogenic mutations, cervical intraepithelial neoplasia, cervical cancer, STDs, or HIV infection can use (U.S. MEC 1) or generally can use (U.S. MEC 2) implants (5); therefore, screening for these conditions is not necessary for the safe initiation of implants.

\section{Routine Follow-Up After Implant Insertion}

These recommendations address when routine follow-up is needed for safe and effective continued use of contraception for healthy women. The recommendations refer to general situations and might vary for different users and different situations. Specific populations who might benefit from more frequent follow-up visits include adolescents, those with certain medical conditions or characteristics, and those with multiple medical conditions.

- Advise the woman to return at any time to discuss side effects or other problems, if she wants to change the method being used, and when it is time to remove or replace the contraceptive method. No routine follow-up visit is required.

- At other routine visits, health care providers seeing implant users should do the following:

- Assess the woman's satisfaction with her contraceptive method and whether she has any concerns about method use.

- Assess any changes in health status, including medications, that would change the appropriateness of the implant for safe and effective continued use based on U.S. MEC (e.g., category 3 and 4 conditions and characteristics).

- Consider assessing weight changes and counseling women who are concerned about weight change perceived to be associated with their contraceptive method.

Comments and Evidence Summary. A systematic review did not identify any evidence regarding whether a routine follow-up visit after initiating an implant improves correct or continued use (122).

\section{Bleeding Irregularities (Including Amenorrhea) During Implant Use}

- Before implant insertion, provide counseling about potential changes in bleeding patterns during implant use. Unscheduled spotting or light bleeding is common with implant use, and some women experience amenorrhea. These bleeding changes are generally not harmful and might or might not decrease with continued implant use. Heavy or prolonged bleeding, unscheduled or menstrual, is uncommon during implant use.

\section{Irregular Bleeding (Spotting, Light Bleeding, or Heavy or Prolonged Bleeding)}

- If clinically indicated, consider an underlying gynecological problem, such as interactions with other medications, an STD, pregnancy, or new pathologic uterine conditions (e.g., polyps or fibroids). If an underlying gynecological problem is found, treat the condition or refer for care.

- If an underlying gynecologic problem is not found and the woman wants treatment, the following treatment options during days of bleeding can be considered:

- NSAIDS for short-term treatment (5-7 days)

- Hormonal treatment (if medically eligible) with lowdose COCs or estrogen for short-term treatment (10-20 days)

- If irregular bleeding persists and the woman finds it unacceptable, counsel her on alternative methods, and offer another method if it is desired.

\section{Amenorrhea}

- Amenorrhea does not require any medical treatment. Provide reassurance.

- If a woman's regular bleeding pattern changes abruptly to amenorrhea, consider ruling out pregnancy if clinically indicated.

- If amenorrhea persists and the woman finds it unacceptable, counsel her on alternative contraceptive methods, and offer another method if it is desired.

Comments and Evidence Summary. During contraceptive counseling and before insertion of the implant, information about common side effects, such as unscheduled spotting or light bleeding and amenorrhea, especially during the first year of use, should be discussed. A pooled analysis of data from 11 clinical trials indicates that a significant proportion of etonogestrel implant users had relatively little bleeding: 22\% of women experienced amenorrhea and $34 \%$ experienced infrequent spotting, although $7 \%$ reported frequent bleeding 
and $18 \%$ reported prolonged bleeding (146). Unscheduled bleeding or amenorrhea is generally not harmful. Enhanced counseling about expected bleeding patterns and reassurance that bleeding irregularities are generally not harmful has been shown to reduce discontinuation in clinical trials with other hormonal contraceptives (i.e., DMPA) $(124,125)$.

A systematic review and four newly published studies examined several medications for the treatment of bleeding irregularities with primarily levonorgestrel contraceptive implants (147-151). Two small studies found significant cessation of bleeding within 7 days of start of treatment among women taking oral celecoxib $(200 \mathrm{mg})$ daily for 5 days or oral mefenamic acid $(500 \mathrm{mg}) 3$ times daily for 5 days compared with placebo $(149,150)$. Differences in bleeding cessation were not found among women with etonogestrel implants taking mifepristone but were found when women with the implants combined mifepristone with either ethinyl estradiol or doxycycline $(151,152)$. Doxycycline alone or in combination with ethinyl estradiol did not improve bleeding cessation among etonogestrel implant users (151). Among LNG implant users, mifepristone reduced the number of bleeding or spotting days but only after 6 months of treatment (153). Evidence also suggests that estrogen (154-156), daily COCs (154), LNG pills (155), tamoxifen (157), or tranexamic acid (158) can reduce the number of bleeding or spotting days during treatment among LNG implant users. In one small study, vitamin E was found to significantly reduce the mean number of bleeding days after the first treatment cycle; however, another larger study reported no significant differences in length of bleeding and spotting episodes with vitamin $\mathrm{E}$ treatment $(159,160)$. Use of aspirin did not result in a significant difference in median length of bleeding or bleeding and spotting episodes after treatment (159). One study among implant users reported a reduction in number of bleeding days after initiating ibuprofen; however, another trial did not demonstrate any significant differences in the number of spotting and bleeding episodes with ibuprofen compared with placebo $(148,155)$.

\section{Injectables}

Progestin-only injectable contraceptives (DMPA, $150 \mathrm{mg}$ intramuscularly or $104 \mathrm{mg}$ subcutaneously) are available in the United States; the only difference between these two formulations is the route of administration. Approximately 6 out of 100 women will become pregnant in the first year of use of DMPA with typical use (14). DMPA is reversible and can be used by women of all ages, including adolescents. DMPA does not protect against STDs; consistent and correct use of male latex condoms reduces the risk for STDs, including HIV.

\section{Initiation of Injectables}

\section{Timing}

- The first DMPA injection can be given at any time if it is reasonably certain that the woman is not pregnant (Box 2).

\section{Need for Back-Up Contraception}

- If DMPA is started within the first 7 days since menstrual bleeding started, no additional contraceptive protection is needed.

- If DMPA is started $>7$ days since menstrual bleeding started, the woman needs to abstain from sexual intercourse or use additional contraceptive protection for the next 7 days.

\section{Special Considerations}

\section{Amenorrhea (Not Postpartum)}

- Timing: The first DMPA injection can be given at any time if it is reasonably certain that the woman is not pregnant (Box 2).

- Need for back-up contraception: The woman needs to abstain from sexual intercourse or use additional contraceptive protection for the next 7 days.

\section{Postpartum (Breastfeeding)}

- Timing: The first DMPA injection can be given at any time, including immediately postpartum (U.S. MEC 2 if $<1$ month postpartum; U.S. MEC 1 if $\geq 1$ month postpartum) if it is reasonably certain that the woman is not pregnant (Box 2).

- Need for back-up contraception: If the woman is $<6$ months postpartum, amenorrheic, and fully or nearly fully breastfeeding (exclusively breastfeeding or the vast majority $[\geq 85 \%$ ] of feeds are breastfeeds) (27), no additional contraceptive protection is needed. Otherwise, a woman who is $\geq 21$ days postpartum and has not experienced return of her menstrual cycle needs to abstain from sexual intercourse or use additional contraceptive protection for the next 7 days. If her menstrual cycles have returned and it has been $>7$ days since menstrual bleeding started, she needs to abstain from sexual intercourse or use additional contraceptive protection for the next 7 days.

\section{Postpartum (Not Breastfeeding)}

- Timing: The first DMPA injection can be given at any time, including immediately postpartum (U.S. MEC 1) if it is reasonably certain that the woman is not pregnant (Box 2).

- Need for back-up contraception: If a woman is $<21$ days postpartum, no additional contraceptive protection is needed. A woman who is $\geq 21$ days postpartum and has 
not experienced return of her menstrual cycle needs to abstain from sexual intercourse or use additional contraceptive protection for the next 7 days. If her menstrual cycles have returned and it has been $>7$ days since menstrual bleeding started, she needs to abstain from sexual intercourse or use additional contraceptive protection for the next 7 days.

\section{Postabortion (Spontaneous or Induced)}

- Timing: The first DMPA injection can be given within the first 7 days, including immediately after the abortion (U.S. MEC 1).

- Need for back-up contraception: The woman needs to abstain from sexual intercourse or use additional contraceptive protection for the next 7 days unless the injection is given at the time of a surgical abortion.

\section{Switching from Another Contraceptive Method}

- Timing: The first DMPA injection can be given immediately if it is reasonably certain that the woman is not pregnant (Box 2). Waiting for her next menstrual cycle is unnecessary.

- Need for back-up contraception: If it has been $>7$ days since menstrual bleeding started, the woman needs to abstain from sexual intercourse or use additional contraceptive protection for the next 7 days.

- Switching from an IUD: If the woman has had sexual intercourse since the start of her current menstrual cycle and it has been $>5$ days since menstrual bleeding started, theoretically, residual sperm might be in the genital tract, which could lead to fertilization if ovulation occurs. A health care provider may consider any of the following options:

- Advise the women to retain the IUD for at least 7 days after the injection and return for IUD removal.

- Advise the woman to abstain from sexual intercourse or use barrier contraception for 7 days before removing the IUD and switching to the new method.

- If the woman cannot return for IUD removal and has not abstained from sexual intercourse or used barrier contraception for 7 days, advise the woman to use ECPs (with the exception of UPA) at the time of IUD removal.

Comments and Evidence Summary. In situations in which the health care provider is uncertain whether the woman might be pregnant, the benefits of starting DMPA likely exceed any risk; therefore, starting DMPA should be considered at any time, with a follow-up pregnancy test in 2-4 weeks. If a woman needs to use additional contraceptive protection when switching to DMPA from another contraceptive method, consider continuing her previous method for 7 days after DMPA injection.
A systematic review identified eight articles examining DMPA initiation on different days of the menstrual cycle (161). Evidence from two studies with small sample sizes indicated that DMPA injections given up to day 7 of the menstrual cycle inhibited ovulation; when DMPA was administered after day 7 , ovulation occurred in some women. Cervical mucus was of poor quality (i.e., not favorable for sperm penetration) in $90 \%$ of women within 24 hours of the injection (Level of evidence: II-2, fair) (162-164). Studies found that use of another contraceptive method until DMPA could be initiated (bridging option) did not help women initiate DMPA and was associated with more unintended pregnancies than immediate receipt of DMPA (165-169) (Level of evidence: I to II-3, fair to poor, indirect).

\section{Examinations and Tests Needed Before Initiation of an Injectable}

Among healthy women, no examinations or tests are needed before initiation of DMPA, although a baseline weight and BMI measurement might be useful to monitor DMPA users over time (Table 3). Women with known medical problems or other special conditions might need additional examinations or tests before being determined to be appropriate candidates for a particular method of contraception. U.S. MEC might be useful in such circumstances (5).

Comments and Evidence Summary. Weight (BMI): Obese women can use (U.S. MEC 1) or generally can use (U.S. MEC 2) DMPA (5); therefore, screening for obesity is not necessary for the safe initiation of DMPA. However, measuring weight and calculating BMI at baseline might be helpful for monitoring any changes and counseling women who might be concerned about weight change perceived to be associated with their contraceptive method. (See guidance on follow-up for DMPA users for evidence on weight gain with DMPA use).

Bimanual examination and cervical inspection: Pelvic examination is not necessary before initiation of DMPA because it does not facilitate detection of conditions for which DMPA would be unsafe. Although women with current breast cancer should not use DMPA (U.S. MEC 4), and women with severe hypertension, heart disease, vascular disease, or certain liver diseases generally should not use DMPA (U.S. MEC 3) (5), none of these conditions are likely to be detected by pelvic examination (145). A systematic review identified two casecontrol studies that compared delayed versus immediate pelvic examination before initiation of hormonal contraceptives, specifically oral contraceptives or DMPA (95). No differences in risk factors for cervical neoplasia, incidence of STDs, incidence of abnormal Papanicolaou smears, or incidence of 
TABLE 3. Classification of examinations and tests needed before depo-medroxyprogesterone acetate initiation

\begin{tabular}{ll}
\hline Examination or test & Class* \\
\hline Examination & \\
Blood pressure & $\mathrm{C}$ \\
Weight (BMI) (weight $[\mathrm{kg}] /$ height $[\mathrm{m}]^{2}$ ) & $\mathrm{C}$ \\
Clinical breast examination & $\mathrm{C}$ \\
Bimanual examination and cervical inspection & \\
Laboratory test & $\mathrm{C}$ \\
Glucose & $\mathrm{C}$ \\
Lipids & $\mathrm{C}$ \\
Liver enzymes & $\mathrm{C}$ \\
Hemoglobin & $\mathrm{C}$ \\
Thrombogenic mutations & $\mathrm{C}$ \\
Cervical cytology (Papanicolaou smear) & $\mathrm{C}$ \\
STD screening with laboratory tests & $\mathrm{C}$ \\
HIV screening with laboratory tests &
\end{tabular}

Abbreviations: $\mathrm{BMI}=$ body mass index; $\mathrm{HIV}=$ human immunodeficiency virus; $\mathrm{STD}=$ sexually transmitted disease; U.S. MEC = U.S. Medical Eligibility Criteria for Contraceptive Use.

* Class A: essential and mandatory in all circumstances for safe and effective use of the contraceptive method. Class B: contributes substantially to safe and effective use, but implementation may be considered within the public health and/or service context; the risk of not performing an examination or test should be balanced against the benefits of making the contraceptive method available. Class C: does not contribute substantially to safe and effective use of the contraceptive method.

t Weight (BMI) measurement is not needed to determine medical eligibility for any methods of contraception because all methods can be used (U.S. MEC 1) or generally can be used (U.S. MEC 2) among obese women (Box 1). However, measuring weight and calculating BMl at baseline might be helpful for monitoring any changes and counseling women who might be concerned about weight change perceived to be associated with their contraceptive method.

abnormal wet mounts were observed (Level of evidence: II-2, fair, direct).

Blood pressure: Women with hypertension generally can use DMPA (U.S. MEC 2), with the exception of women with severe hypertension or vascular disease, who generally should not use DMPA (U.S. MEC 3) (5). Screening for hypertension before initiation of DMPA is not necessary because of the low prevalence of undiagnosed severe hypertension and the high likelihood that women with these conditions already would have had them diagnosed. A systematic review did not identify any evidence regarding outcomes among women who were screened versus not screened with a blood pressure measurement before initiation of progestin-only contraceptives (170). The prevalence of undiagnosed hypertension among women of reproductive age is low. During 2009-2012 among women aged 20-44 years in the United States, the prevalence of hypertension was $8.7 \%$ (84). During 1999-2008, the percentage of women aged 20-44 years with undiagnosed hypertension was $1.9 \%$ (85).

Glucose: Although women with complicated diabetes generally should not use DMPA (U.S. MEC 3) (5), screening for diabetes before initiation of DMPA is not necessary because of the low prevalence of undiagnosed diabetes and the high likelihood that women with complicated diabetes would already have had the condition diagnosed. A systematic review did not identify any evidence regarding outcomes among women who were screened versus not screened with glucose measurement before initiation of hormonal contraceptives (57). The prevalence of diabetes among women of reproductive age is low. During 2009-2012 among women aged 20-44 years in the United States, the prevalence of diabetes was 3.3\% (84). During 1999-2008, the percentage of women aged 20-44 years with undiagnosed diabetes was $0.5 \%$ (85). Although hormonal contraceptives can have some adverse effects on glucose metabolism in healthy and diabetic women, the overall clinical effect is minimal (171-177).

Lipids: Screening for dyslipidemias is not necessary for the safe initiation of injectables because of the low prevalence of undiagnosed disease in women of reproductive age and the low likelihood of clinically significant changes with use of hormonal contraceptives. A systematic review did not identify any evidence regarding outcomes among women who were screened versus not screened with lipid measurement before initiation of hormonal contraceptives (57). During 2009-2012 among women aged 20-44 years in the United States, 7.6\% had high cholesterol, defined as total serum cholesterol $\geq 240 \mathrm{mg} / \mathrm{dL}$ (84). During 1999-2008, the prevalence of undiagnosed hypercholesterolemia among women aged 20-44 years was approximately $2 \%$ (85). Studies have shown mixed results about the effects of hormonal methods on lipid levels among both healthy women and women with baseline lipid abnormalities, and the clinical significance of these changes is unclear (86-89).

Liver enzymes: Although women with certain liver diseases generally should not use DMPA (U.S. MEC 3) (5), screening for liver disease before initiation of DMPA is not necessary because of the low prevalence of these conditions and the high likelihood that women with liver disease already would have had the condition diagnosed. A systematic review did not identify any evidence regarding outcomes among women who were screened versus not screened with liver enzyme tests before initiation of hormonal contraceptives (57). In 2012, among U.S. women, the percentage with liver disease (not further specified) was $1.3 \%$ (90). In 2013, the incidence of acute hepatitis A, B, or $\mathrm{C}$ was $\leq 1$ per 100,000 U.S. population (91). During 2002-2011, the incidence of liver carcinoma among U.S. women was approximately 3.7 per 100,000 population (92). Because estrogen and progestins are metabolized in the liver, the use of hormonal contraceptives among women with liver disease might, theoretically, be a concern. The use of hormonal contraceptives, specifically COCs and POPs, does not affect disease progression or severity in women with hepatitis, cirrhosis, or benign focal nodular hyperplasia $(93,94)$, although evidence is limited and no evidence exists for DMPA. 
Clinical breast examination: Although women with current breast cancer should not use DMPA (U.S. MEC 4) (5), screening asymptomatic women with a clinical breast examination before initiating DMPA is not necessary because of the low prevalence of breast cancer among women of reproductive age. A systematic review did not identify any evidence regarding outcomes among women who were screened versus not screened with a clinical breast examination before initiation of hormonal contraceptives (95). The incidence of breast cancer among women of reproductive age in the United States is low. In 2012, the incidence of breast cancer among women aged 20-49 years was approximately 70.7 per 100,000 women (96).

Other screening: Women with anemia, thrombogenic mutations, cervical intraepithelial neoplasia, cervical cancer, HIV infection, or other STDs can use (U.S. MEC 1) or generally can use (U.S. MEC 2) DMPA (5); therefore, screening for these conditions is not necessary for the safe initiation of DMPA.

\section{Routine Follow-Up After Injectable Initiation}

These recommendations address when routine follow-up is recommended for safe and effective continued use of contraception for healthy women. The recommendations refer to general situations and might vary for different users and different situations. Specific populations who might benefit from frequent follow-up visits include adolescents, those with certain medical conditions or characteristics, and those with multiple medical conditions.

- Advise the woman to return at any time to discuss side effects or other problems, if she wants to change the method being used, and when it is time for reinjection. No routine follow-up visit is required.

- At other routine visits, health care providers seeing injectable users should do the following:

- Assess the woman's satisfaction with her contraceptive method and whether she has any concerns about method use.

- Assess any changes in health status, including medications, that would change the appropriateness of the injectable for safe and effective continued use based on U.S. MEC (e.g., category 3 and 4 conditions and characteristics).

- Consider assessing weight changes and counseling women who are concerned about weight change perceived to be associated with their contraceptive method.

Comments and Evidence Summary. Although no evidence exists regarding whether a routine follow-up visit after initiating DMPA improves correct or continued use, monitoring weight or BMI change over time is important for DMPA users.
A systematic review identified a limited body of evidence that examined whether weight gain in the few months after DMPA initiation predicted future weight gain (123). Two studies found significant differences in weight gain or BMI at follow-up periods ranging from 12 to 36 months between early weight gainers (i.e., those who gained $>5 \%$ of their baseline body weight within 6 months after initiation) and those who were not early weight gainers $(178,179)$. The differences between groups were more pronounced at 18, 24, and 36 months than at 12 months. One study found that most adolescent DMPA users who had gained $>5 \%$ of their baseline weight by 3 months gained even more weight by 12 months (180) (Level of evidence: II-2, fair, to II-3, fair, direct).

\section{Timing of Repeat Injections}

\section{Reinjection Interval}

- Provide repeat DMPA injections every 3 months (13 weeks).

\section{Special Considerations}

\section{Early Injection}

- The repeat DMPA injection can be given early when necessary.

\section{Late Injection}

- The repeat DMPA injection can be given up to 2 weeks late (15 weeks from the last injection) without requiring additional contraceptive protection.

- If the woman is $>2$ weeks late ( $>15$ weeks from the last injection) for a repeat DMPA injection, she can have the injection if it is reasonably certain that she is not pregnant (Box 2). She needs to abstain from sexual intercourse or use additional contraceptive protection for the next 7 days. She might consider the use of emergency contraception (with the exception of UPA) if appropriate.

Comments and Evidence Summary. No time limits exist for early injections; injections can be given when necessary (e.g., when a woman cannot return at the routine interval). WHO has extended the time that a woman can have a late reinjection (i.e., grace period) for DMPA use from 2 weeks to 4 weeks on the basis of data from one study showing low pregnancy rates through 4 weeks; however, the CDC expert group did not consider the data to be generalizable to the United States because a large proportion of women in the study were breastfeeding. Therefore, U.S. SPR recommends a grace period of 2 weeks.

A systematic review identified 12 studies evaluating time to pregnancy or ovulation after the last injection of DMPA (181). Although pregnancy rates were low during the 2-week interval following the reinjection date and for 4 weeks following the 
reinjection date, data were sparse, and one study included a large proportion of breastfeeding women (182-184). Studies also indicated a wide variation in time to ovulation after the last DMPA injection, with the majority ranging from 15 to 49 weeks from the last injection (185-193) (Level of evidence: level II-2, fair, direct).

\section{Bleeding Irregularities (Including Amenorrhea) During Injectable Use}

- Before DMPA initiation, provide counseling about potential changes in bleeding patterns during DMPA use. Amenorrhea and unscheduled spotting or light bleeding is common with DMPA use, and heavy or prolonged bleeding can occur with DMPA use. These bleeding irregularities are generally not harmful and might decrease with continued DMPA use.

\section{Unscheduled Spotting or Light Bleeding}

- If clinically indicated, consider an underlying gynecological problem, such as interactions with other medications, an STD, pregnancy, or new pathologic uterine conditions (e.g., polyps or fibroids). If an underlying gynecological problem is found, treat the condition or refer for care.

- If an underlying gynecologic problem is not found and the woman wants treatment, the following treatment option during days of bleeding can be considered:

- NSAIDs for short-term treatment (5-7 days)

- If unscheduled spotting or light bleeding persists and the woman finds it unacceptable, counsel her on alternative contraceptive methods, and offer another method if it is desired.

\section{Heavy or Prolonged Bleeding}

- If clinically indicated, consider an underlying gynecological problem, such as interactions with other medications, an STD, pregnancy, or new pathologic uterine conditions (such as fibroids or polyps). If an underlying gynecologic problem is identified, treat the condition or refer for care.

- If an underlying gynecologic problem is not found and the woman wants treatment, the following treatment options during days of bleeding can be considered:

- NSAIDS for short-term treatment (5-7 days)

- Hormonal treatment (if medically eligible) with lowdose COCs or estrogen for short-term treatment (10-20 days)

- If heavy or prolonged bleeding persists and the woman finds it unacceptable, counsel her on alternative contraceptive methods, and offer another method if it is desired.

\section{Amenorrhea}

- Amenorrhea does not require any medical treatment. Provide reassurance.

- If a woman's regular bleeding pattern changes abruptly to amenorrhea, consider ruling out pregnancy if clinically indicated.

- If amenorrhea persists and the woman finds it unacceptable, counsel her on alternative contraceptive methods, and offer another method if it is desired.

Comments and Evidence Summary. During contraceptive counseling and before initiation of DMPA, information about common side effects such as irregular bleeding should be discussed. Unscheduled bleeding or spotting is common with DMPA use (194). In addition, amenorrhea is common after $\geq 1$ years of continuous use $(194,195)$. These bleeding irregularities are generally not harmful. Enhanced counseling among DMPA users detailing expected bleeding patterns and reassurance that these irregularities generally are not harmful has been shown to reduce DMPA discontinuation in clinical trials $(124,125)$.

A systematic review, as well as two additional studies, examined the treatment of bleeding irregularities during DMPA use (195-197). Two small studies found significant cessation of bleeding within 7 days of starting treatment among women taking valdecoxib for 5 days or mefenamic acid for 5 days compared with placebo $(198,199)$. Treatment with ethinyl estradiol was found to stop bleeding better than placebo during the treatment period, although rates of discontinuation were high and safety outcomes were not examined (200). In one small study among DMPA users who had been experiencing amenorrhea for 2 months, treatment with COCs was found to alleviate amenorrhea better than placebo (201). No studies examined the effects of aspirin on bleeding irregularities among DMPA users.

\section{Combined Hormonal Contraceptives}

Combined hormonal contraceptives contain both estrogen and a progestin and include 1) COCs (various formulations), 2) a transdermal contraceptive patch (which releases $150 \mu \mathrm{g}$ of norelgestromin and $20 \mu$ g ethinyl estradiol daily), and 3) a vaginal contraceptive ring (which releases $120 \mu \mathrm{g}$ etonogestrel and $15 \mu \mathrm{g}$ ethinyl estradiol daily). Approximately 9 out of 100 women become pregnant in the first year of use with combined hormonal contraceptives with typical use (14). These methods are reversible and can be used by women of all ages. Combined hormonal contraceptives are generally used for 
21-24 consecutive days, followed by 4-7 hormone-free days (either no use or placebo pills). These methods are sometimes used for an extended period with infrequent or no hormonefree days. Combined hormonal contraceptives do not protect against STDs; consistent and correct use of male latex condoms reduces the risk for STDs, including HIV.

\section{Initiation of Combined Hormonal Contraceptives}

\section{Timing}

- Combined hormonal contraceptives can be initiated at any time if it is reasonably certain that the woman is not pregnant (Box 2).

\section{Need for Back-Up Contraception}

- If combined hormonal contraceptives are started within the first 5 days since menstrual bleeding started, no additional contraceptive protection is needed.

- If combined hormonal contraceptives are started $>5$ days since menstrual bleeding started, the woman needs to abstain from sexual intercourse or use additional contraceptive protection for the next 7 days.

\section{Special Considerations}

\section{Amenorrhea (Not Postpartum)}

- Timing: Combined hormonal contraceptives can be started at any time if it is reasonably certain that the woman is not pregnant (Box 2).

- Need for back-up contraception: The woman needs to abstain from sexual intercourse or use additional contraceptive protection for the next 7 days.

\section{Postpartum (Breastfeeding)}

- Timing: Combined hormonal contraceptives can be started when the woman is medically eligible to use the method (5) and if it is reasonably certain that she is not pregnant. (Box 2).

- Postpartum women who are breastfeeding should not use combined hormonal contraceptives during the first 3 weeks after delivery (U.S. MEC 4) because of concerns about increased risk for venous thromboembolism and generally should not use combined hormonal contraceptives during the fourth week postpartum (U.S. MEC 3) because of concerns about potential effects on breastfeeding performance. Postpartum breastfeeding women with other risk factors for venous thromboembolism generally should not use combined hormonal contraceptives 4-6 weeks after delivery (U.S. MEC 3).
- Need for back-up contraception: If the woman is $<6$ months postpartum, amenorrheic, and fully or nearly fully breastfeeding (exclusively breastfeeding or the vast majority $[\geq 85 \%]$ of feeds are breastfeeds) (27), no additional contraceptive protection is needed. Otherwise, a woman who is $\geq 21$ days postpartum and has not experienced return of her menstrual cycle needs to abstain from sexual intercourse or use additional contraceptive protection for the next 7 days. If her menstrual cycles have returned and it has been $>5$ days since menstrual bleeding started, she needs to abstain from sexual intercourse or use additional contraceptive protection for the next 7 days.

\section{Postpartum (Not Breastfeeding)}

- Timing: Combined hormonal contraceptives can be started when the woman is medically eligible to use the method (5) and if it is reasonably certain that the she is not pregnant (Box 2).

- Postpartum women should not use combined hormonal contraceptives during the first 3 weeks after delivery (U.S. MEC 4) because of concerns about increased risk for venous thromboembolism. Postpartum women with other risk factors for venous thromboembolism generally should not use combined hormonal contraceptives 3-6 weeks after delivery (U.S. MEC 3).

- Need for back-up contraception: If a woman is $<21$ days postpartum, no additional contraceptive protection is needed. A woman who is $\geq 21$ days postpartum and whose menstrual cycles have not returned needs to abstain from sexual intercourse or use additional contraceptive protection for the next 7 days. If her menstrual cycles have returned and it has been $>5$ days since menstrual bleeding started, she needs to abstain from sexual intercourse or use additional contraceptive protection for the next 7 days.

\section{Postabortion (Spontaneous or Induced)}

- Timing: Combined hormonal contraceptives can be started within the first 7 days following first-trimester or second-trimester abortion, including immediately postabortion (U.S. MEC 1).

- Need for back-up contraception: The woman needs to abstain from sexual intercourse or use additional contraceptive protection for the next 7 days unless combined hormonal contraceptives are started at the time of a surgical abortion.

\section{Switching from Another Contraceptive Method}

- Timing: Combined hormonal contraceptives can be started immediately if it is reasonably certain that the 
woman is not pregnant (Box 2). Waiting for her next menstrual cycle is unnecessary.

- Need for back-up contraception: If it has been $>5$ days since menstrual bleeding started, she needs to abstain from sexual intercourse or use additional contraceptive protection for the next 7 days.

- Switching from an IUD: If the woman has had sexual intercourse since the start of her current menstrual cycle and it has been $>5$ days since menstrual bleeding started, theoretically, residual sperm might be in the genital tract, which could lead to fertilization if ovulation occurs. A health care provider may consider any of the following options:

- Advise the women to retain the IUD for at least 7 days after combined hormonal contraceptives are initiated and return for IUD removal.

- Advise the woman to abstain from sexual intercourse or use barrier contraception for 7 days before removing the IUD and switching to the new method.

- If the woman cannot return for IUD removal and has not abstained from sexual intercourse or used barrier contraception for 7 days, advise the woman to use ECPs at the time of IUD removal. Combined hormonal contraceptives can be started immediately after use of ECPs (with the exception of UPA). Combined hormonal contraceptives can be started no sooner than 5 days after use of UPA.

Comments and Evidence Summary. In situations in which the health care provider is uncertain whether the woman might be pregnant, the benefits of starting combined hormonal contraceptives likely exceed any risk; therefore, starting combined hormonal contraceptives should be considered at any time, with a follow-up pregnancy test in $2-4$ weeks. If a woman needs to use additional contraceptive protection when switching to combined hormonal contraceptives from another contraceptive method, consider continuing her previous method for 7 days after starting combined hormonal contraceptives.

A systematic review of 18 studies examined the effects of starting combined hormonal contraceptives on different days of the menstrual cycle (202). Overall, the evidence suggested that pregnancy rates did not differ by the timing of combined hormonal contraceptive initiation (169,203-205) (Level of evidence: I to II-3, fair, indirect). The more follicular activity that occurred before starting COCs, the more likely ovulation was to occur; however, no ovulations occurred when COCs were started at a follicle diameter of $10 \mathrm{~mm}$ (mean cycle day 7.6) or when the ring was started at $13 \mathrm{~mm}$ (median cycle day 11) (206-215) (Level of evidence: I to II-3, fair, indirect). Bleeding patterns and other side effects did not vary with the timing of combined hormonal contraceptive initiation $(204,205,216-220)$ (Level of evidence: I to II-2, good to poor, direct). Although continuation rates of combined hormonal contraceptives were initially improved by the "quick start" approach (i.e., starting on the day of the visit), the advantage disappeared over time (203,204,216-221) (Level of evidence: I to II-2, good to poor, direct).

\section{Examinations and Test Needed Before Initiation of Combined Hormonal Contraceptives}

Among healthy women, few examinations or tests are needed before initiation of combined hormonal contraceptives (Table 4). Blood pressure should be measured before initiation of combined hormonal contraceptives. Baseline weight and BMI measurements might be useful for monitoring combined hormonal contraceptive users over time. Women with known medical problems or other special conditions might need additional examinations or tests before being determined to be appropriate candidates for a particular method of contraception. U.S. MEC might be useful in such circumstances (5).

Comments and Evidence Summary. Blood pressure: Women who have more severe hypertension (systolic pressure of $\geq 160 \mathrm{mmHg}$ or diastolic pressure of $\geq 100 \mathrm{~mm} \mathrm{Hg}$ ) or vascular disease should not use combined hormonal contraceptives (U.S. MEC 4), and women who have less severe hypertension (systolic pressure of 140-159 mm Hg or diastolic pressure of $90-99 \mathrm{~mm} \mathrm{Hg}$ ) or adequately controlled hypertension generally should not use combined hormonal contraceptives (U.S. MEC 3) (5). Therefore, blood pressure should be evaluated before initiating combined hormonal contraceptives. In instances in which blood pressure cannot be measured by a provider, blood pressure measured in other settings can be reported by the woman to her provider. Evidence suggests that cardiovascular outcomes are worse among women who did not have their blood pressure measured before initiating COCs. A systematic review identified six articles from three studies that reported cardiovascular outcomes among women who had blood pressure measurements and women who did not have blood pressure measurements before initiating COCs (170). Three case-control studies showed that women who did not have blood pressure measurements before initiating COCs had a higher risk for acute myocardial infarction than women who did have blood pressure measurements (222-224). Two case-control studies showed that women who did not have blood pressure measurements before initiating COCs had a higher risk for ischemic stroke than women who did have blood pressure measurements $(225,226)$. One case-control study showed no difference in the risk for hemorrhagic stroke among women who initiated COCs regardless of whether their 
TABLE 4. Classification of examinations and tests needed before combined hormonal contraceptive initiation

\begin{tabular}{lc}
\hline Examination or test & Class* \\
\hline Examination & \\
Blood pressure & $\mathrm{A}^{\dagger}$ \\
Weight $(\mathrm{BMI})\left(\right.$ weight $[\mathrm{kg}] /$ height $[\mathrm{m}]^{2}$ ) & $-\mathrm{C}^{\text {C }}$ \\
Clinical breast examination & $\mathrm{C}$ \\
Bimanual examination and cervical inspection & \\
Laboratory test & $\mathrm{C}$ \\
Glucose & $\mathrm{C}$ \\
Lipids & $\mathrm{C}$ \\
Liver enzymes & $\mathrm{C}$ \\
Hemoglobin & $\mathrm{C}$ \\
Thrombogenic mutations & $\mathrm{C}$ \\
Cervical cytology (Papanicolaou smear) & $\mathrm{C}$ \\
STD screening with laboratory tests & $\mathrm{C}$ \\
HIV screening with laboratory tests & \\
\hline
\end{tabular}

Abbreviations: $\mathrm{BMI}=$ body mass index; $\mathrm{HIV}=$ human immunodeficiency virus; $\mathrm{STD}=$ sexually transmitted disease; U.S. MEC = U.S. Medical Eligibility Criteria for Contraceptive Use.

* Class A: essential and mandatory in all circumstances for safe and effective use of the contraceptive method. Class B: contributes substantially to safe and effective use, but implementation may be considered within the public health and/or service context; the risk of not performing an examination or test should be balanced against the benefits of making the contraceptive method available. Class C: does not contribute substantially to safe and effective use of the contraceptive method.

† In instances in which blood pressure cannot be measured by a provider, blood pressure measured in other settings can be reported by the woman to her provider.

$\S$ Weight (BMI) measurement is not needed to determine medical eligibility for any methods of contraception because all methods can be used (U.S. MEC 1) or generally can be used (U.S. MEC 2) among obese women (Box 1). However, measuring weight and calculating BMl at baseline might be helpful for monitoring any changes and counseling women who might be concerned about weight change perceived to be associated with their contraceptive method.

blood pressure was measured (227). Studies that examined hormonal contraceptive methods other than COCs were not identified (Level of evidence: II-2, fair, direct).

Weight (BMI): Obese women generally can use combined hormonal contraceptives (U.S. MEC 2) (5); therefore, screening for obesity is not necessary for the safe initiation of combined hormonal contraceptives. However, measuring weight and calculating BMI at baseline might be helpful for monitoring any changes and counseling women who might be concerned about weight change perceived to be associated with their contraceptive method.

Bimanual examination and cervical inspection: Pelvic examination is not necessary before initiation of combined hormonal contraceptives because it does not facilitate detection of conditions for which hormonal contraceptives would be unsafe. Women with certain conditions such as current breast cancer, severe hypertension or vascular disease, heart disease, migraine headaches with aura, and certain liver diseases, as well as women aged $\geq 35$ years and who smoke $\geq 15$ cigarettes per day, should not use (U.S. MEC 4) or generally should not use (U.S. MEC 3) combined hormonal contraceptives (5); however, none of these conditions are likely to be detected by pelvic examination (145). A systematic review identified two case-control studies that compared delayed and immediate pelvic examination before initiation of hormonal contraceptives, specifically oral contraceptives or DMPA (95). No differences in risk factors for cervical neoplasia, incidence of STDs, incidence of abnormal Papanicolaou smears, or incidence of abnormal wet mounts were found (Level of evidence: Level II-2 fair, direct).

Glucose: Although women with complicated diabetes should not use (U.S. MEC 4) or generally should not use (U.S. MEC 3) combined hormonal contraceptives, depending on the severity of the condition (5), screening for diabetes before initiation of hormonal contraceptives is not necessary because of the low prevalence of undiagnosed diabetes and the high likelihood that women with complicated diabetes already would have had the condition diagnosed. A systematic review did not identify any evidence regarding outcomes among women who were screened versus not screened with glucose measurement before initiation of hormonal contraceptives (57). The prevalence of diabetes among women of reproductive age is low. During 2009-2012 among women aged 20-44 years in the United States, the prevalence of diabetes was 3.3\% (84). During 1999-2008, the percentage of women aged 20-44 years with undiagnosed diabetes was $0.5 \%$ (85). Although hormonal contraceptives can have some adverse effects on glucose metabolism in healthy and diabetic women, the overall clinical effect is minimal (171-177).

Lipids: Screening for dyslipidemias is not necessary for the safe initiation of combined hormonal contraceptives because of the low prevalence of undiagnosed disease in women of reproductive age and the low likelihood of clinically significant changes with use of hormonal contraceptives. A systematic review did not identify any evidence regarding outcomes among women who were screened versus not screened with lipid measurement before initiation of hormonal contraceptives (57). During 2009-2012 among women aged 20-44 years in the United States, 7.6\% had high cholesterol, defined as total serum cholesterol $\geq 240 \mathrm{mg} / \mathrm{dL}$ (84). During 1999-2008, the prevalence of undiagnosed hypercholesterolemia among women aged 20-44 years was approximately 2\% (85). A systematic review identified few studies, all of poor quality, that suggest that women with known dyslipidemias using combined hormonal contraceptives might be at increased risk for myocardial infarction, cerebrovascular accident, or venous thromboembolism compared with women without dyslipidemias; no studies were identified that examined risk for pancreatitis among women with known dyslipidemias using combined hormonal contraceptives (89). Studies have shown mixed results regarding the effects of hormonal contraceptives on lipid levels among both healthy women and women with 
baseline lipid abnormalities, and the clinical significance of these changes is unclear $(86-89)$.

Liver enzymes: Although women with certain liver diseases should not use (U.S. MEC 4) or generally should not use (U.S. MEC 3) combined hormonal contraceptives (5), screening for liver disease before initiation of combined hormonal contraceptives is not necessary because of the low prevalence of these conditions and the high likelihood that women with liver disease already would have had the condition diagnosed. A systematic review did not identify any evidence regarding outcomes among women who were screened versus not screened with liver enzyme tests before initiation of hormonal contraceptives (57). In 2012, among U.S. women, the percentage with liver disease (not further specified) was $1.3 \%$ (90). In 2013, the incidence of acute hepatitis A, B, or $\mathrm{C}$ was $\leq 1$ per 100,000 U.S. population (91). During 2002-2011, the incidence of liver carcinoma among U.S. women was approximately 3.7 per 100,000 population (92). Because estrogen and progestins are metabolized in the liver, the use of hormonal contraceptives among women with liver disease might, theoretically, be a concern. The use of hormonal contraceptives, specifically COCs and POPs, does not affect disease progression or severity in women with hepatitis, cirrhosis, or benign focal nodular hyperplasia $(93,94)$, although evidence is limited; no evidence exists for other types of combined hormonal contraceptives.

Thrombogenic mutations: Women with thrombogenic mutations should not use combined hormonal contraceptives (U.S. MEC 4) (5) because of the increased risk for venous thromboembolism (228). However, studies have shown that universal screening for thrombogenic mutations before initiating COCs is not cost-effective because of the rarity of the conditions and the high cost of screening (229-231).

Clinical breast examination: Although women with current breast cancer should not use combined hormonal contraceptives (U.S. MEC 4) (5), screening asymptomatic women with a clinical breast examination before initiating combined hormonal contraceptives is not necessary because of the low prevalence of breast cancer among women of reproductive age. A systematic review did not identify any evidence regarding outcomes among women who were screened versus not screened with a breast examination before initiation of hormonal contraceptives (95). The incidence of breast cancer among women of reproductive age in the United States is low. In 2012, the incidence of breast cancer among women aged 20-49 years was approximately 70.7 per 100,000 women (96).

Other screening: Women with anemia, cervical intraepithelial neoplasia, cervical cancer, HIV infection, or other STDs can use (U.S. MEC 1) or generally can use (U.S. MEC 2) combined hormonal contraceptives (5); therefore, screening for these conditions is not necessary for the safe initiation of combined hormonal contraceptives.

\section{Number of Pill Packs that Should Be Provided at Initial and Return Visits}

- At the initial and return visits, provide or prescribe up to a 1-year supply of COCs (e.g., 13 28-day pill packs), depending on the woman's preferences and anticipated use.

- A woman should be able to obtain COCs easily in the amount and at the time she needs them.

Comments and Evidence Summary. The more pill packs given up to 13 cycles, the higher the continuation rates. Restricting the number of pill packs distributed or prescribed can result in unwanted discontinuation of the method and increased risk for pregnancy.

A systematic review of the evidence suggested that providing a greater number of pill packs was associated with increased continuation (232). Studies that compared provision of one versus 12 packs, one versus 12 or 13 packs, or three versus seven packs found increased continuation of pill use among women provided with more pill packs (233-235). However, one study found no difference in continuation when patients were provided one and then three packs versus four packs all at once (236). In addition to continuation, a greater number of pills packs provided was associated with fewer pregnancy tests, fewer pregnancies, and lower cost per client. However, a greater number of pill packs (i.e., 13 packs versus three packs) also was associated with increased pill wastage in one study (234) (Level of evidence: I to II-2, fair, direct).

\section{Routine Follow-Up After Combined Hormonal Contraceptive Initiation}

These recommendations address when routine follow-up is recommended for safe and effective continued use of contraception for healthy women. The recommendations refer to general situations and might vary for different users and different situations. Specific populations who might benefit from more frequent follow-up visits include adolescents, those with certain medical conditions or characteristics, and those with multiple medical conditions.

- Advise the woman to return at any time to discuss side effects or other problems or if she wants to change the method being used. No routine follow-up visit is required.

- At other routine visits, health care providers seeing combined hormonal contraceptive users should do the following: 
- Assess the woman's satisfaction with her contraceptive method and whether she has any concerns about method use.

- Assess any changes in health status, including medications, that would change the appropriateness of combined hormonal contraceptives for safe and effective continued use based on U.S. MEC (e.g., category 3 and 4 conditions and characteristics).

- Assess blood pressure.

- Consider assessing weight changes and counseling women who are concerned about weight change perceived to be associated with their contraceptive method.

Comments and Evidence Summary. No evidence exists regarding whether a routine follow-up visit after initiating combined hormonal contraceptives improves correct or continued use. Monitoring blood pressure is important for combined hormonal contraceptive users. Health care providers might consider recommending women obtain blood pressure measurements in other settings.

A systematic review identified five studies that examined the incidence of hypertension among women who began using a COC versus those who started a nonhormonal method of contraception or a placebo (123). Few women developed hypertension after initiating COCs, and studies examining increases in blood pressure after COC initiation found mixed results. No studies were identified that examined changes in blood pressure among patch or vaginal ring users (Level of evidence: I, fair, to II-2, fair, indirect).

\section{Late or Missed Doses and Side Effects from Combined Hormonal Contraceptive Use}

For the following recommendations, a dose is considered late when $<24$ hours have elapsed since the dose should have been taken. A dose is considered missed if $\geq 24$ hours have elapsed since the dose should have been taken. For example, if a COC pill was supposed to have been taken on Monday at 9:00 a.m. and is taken at 11:00 a.m., the pill is late; however, by Tuesday morning at 11:00 a.m., Monday's 9:00 a.m. pill has been missed and Tuesday's 9:00 a.m. pill is late. For COCs, the recommendations only apply to late or missed hormonally active pills and not to placebo pills. Recommendations are provided for late or missed pills (Figure 2), the patch (Figure 3), and the ring (Figure 4).

Comments and Evidence Summary. Inconsistent or incorrect use of combined hormonal contraceptives is a major cause of combined hormonal contraceptive failure. Extending the hormone-free interval is considered to be a particularly risky time to miss combined hormonal contraceptives. Seven days of continuous combined hormonal contraceptive use is deemed necessary to reliably prevent ovulation. The recommendations reflect a balance between simplicity and precision of science. Women who frequently miss COCs or experience other usage errors with combined hormonal patch or combined vaginal ring should consider an alternative contraceptive method that is less dependent on the user to be effective (e.g., IUD, implant, or injectable).

A systematic review identified 36 studies that examined measures of contraceptive effectiveness of combined hormonal contraceptives during cycles with extended hormone-free intervals, shortened hormone-free intervals, or deliberate nonadherence on days not adjacent to the hormone-free interval (237). Most of the studies examined COCs $(215,238$ 265), two examined the combined hormonal patch $(259,266)$, and six examined the combined vaginal ring $(211,267-271)$. No direct evidence on the effect of missed pills on the risk for pregnancy was found. Studies of women deliberately extending the hormone-free interval up to 14 days found wide variability in the amount of follicular development and occurrence of ovulation $(241,244,246,247,249,250,252-255)$; in general, the risk for ovulation was low, and among women who did ovulate, cycles were usually abnormal. In studies of women who deliberately missed pills on various days during the cycle not adjacent to the hormone-free interval, ovulation occurred infrequently $(239,245-247,255,256,258,259)$. Studies comparing 7-day hormone-free intervals with shorter hormone-free intervals found lower rates of pregnancy $(238,242,251,257)$ and significantly greater suppression of ovulation $(240,250,261-263,265)$ among women with shorter intervals in all but one study (260), which found no difference. Two studies that compared $30-\mu \mathrm{g}$ ethinyl estradiol pills with $20-\mu \mathrm{g}$ ethinyl estradiol pills showed more follicular activity when 20- $\mu$ g ethinyl estradiol pills were missed $(241,244)$. In studies examining the combined vaginal ring, three studies found that nondeliberate extension of the hormone-free interval for 24 to $<48$ hours from the scheduled period did not increase the risk for pregnancy $(267,268,270)$; one study found that ring insertion after a deliberately extended hormone-free interval that allowed a 13-mm follicle to develop interrupted ovarian function and further follicular growth (211); and one study found that inhibition of ovulation was maintained after deliberately forgetting to remove the ring for up to 2 weeks after normal ring use (271). In studies examining the combined hormonal patch, one study found that missing 1-3 consecutive days before patch replacement (either wearing one patch 3 days longer before replacement or going 3 days without a patch before replacing the next patch) on days not adjacent to the patch-free interval resulted in little follicular activity and low risk for ovulation (259), and one pharmacokinetic study found that serum levels of 
FIGURE 2. Recommended actions after late or missed combined oral contraceptives

If one hormonal pill is late:

$(<24$ hours since a pill should have been taken)
If one hormonal pill has been missed: ( 24 to $<48$ hours since a pill should have been taken)

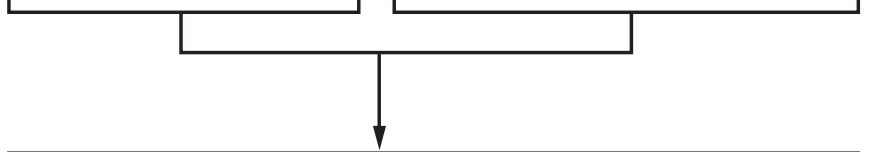

- Take the late or missed pill as soon as possible.

- Continue taking the remaining pills at the usual time (even if it

means taking two pills on the same day).

- No additional contraceptive protection is needed.

- Emergency contraception is not usually needed but can be considered (with the exception of UPA) if hormonal pills were missed earlier in the cycle or in the last week of the previous cycle.

If two or more consecutive hormonal pills have been missed:

( $\geq 48$ hours since a pill should have been taken)

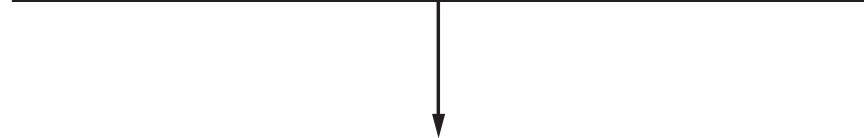

- Take the most recent missed pill as soon as possible. (Any other missed pills should be discarded.)

- Continue taking the remaining pills at the usual time (even if it means taking two pills on the same day).

- Use back-up contraception (e.g., condoms) or avoid sexual intercourse until hormonal pills have been taken for 7 consecutive days.

- If pills were missed in the last week of hormonal pills (e.g., days 15-21 for 28-day pill packs):

o Omit the hormone-free interval by finishing the hormonal pills in the current pack and starting a new pack the next day. o If unable to start a new pack immediately, use back-up contraception (e.g., condoms) or avoid sexual intercourse until hormonal pills from a new pack have been taken for 7 consecutive days.

- Emergency contraception should be considered (with the exception of UPA) if hormonal pills were missed during the first week and unprotected sexual intercourse occurred in the previous 5 days.

- Emergency contraception may also be considered (with the exception of UPA) at other times as appropriate.

Abbreviation: UPA = ulipristal acetate.

FIGURE 3. Recommended actions after delayed application or detachment* with combined hormonal patch

Delayed application or detachment for $<48$ hours since a patch

should have been applied or reattached
Delayed application or detachment for $\geq 48$ hours since a patch

should have been applied or reattached
- Apply a new patch as soon as possible. (If detachment occurred $<24$ hours since the patch was applied, try to reapply the patch or replace with a new patch.)
- Keep the same patch change day.
- No additional contraceptive protection is needed.
- Emergency contraception is not usually needed but can be considered (with the exception of UPA) if delayed application or detachment occurred earlier in the cycle or in the last week of the previous cycle.

- Apply a new patch as soon as possible.
- Keep the same patch change day.
- Use back-up contraception (e.g., condoms) or avoid sexual
intercourse until a patch has been worn for 7 consecutive days.
- If the delayed application or detachment occurred in the third
patch week:
o Omit the hormone-free week by finishing the third week of
patch use (keeping the same patch change day) and starting a
new patch immediately;
o If unable to start a new patch immediately, use back-up
contraception (e.g., condoms) or avoid sexual intercourse until a
new patch has been worn for 7 consecutive days.
- Emergency contraception should be considered (with the exception
of UPA) if the delayed application or detachment occurred within
the first week of patch use and unprotected sexual intercourse
occurred in the previous 5 days.
- Emergency contraception may also be considered (with the
exception of UPA) at other times as appropriate.

Abbreviation: UPA = ulipristal acetate.

* If detachment takes place but the woman is unsure when the detachment occurred, consider the patch to have been detached for $\geq 48$ hours since a patch should have been applied or reattached. 
FIGURE 4. Recommended actions after delayed insertion or reinsertion* with combined vaginal ring

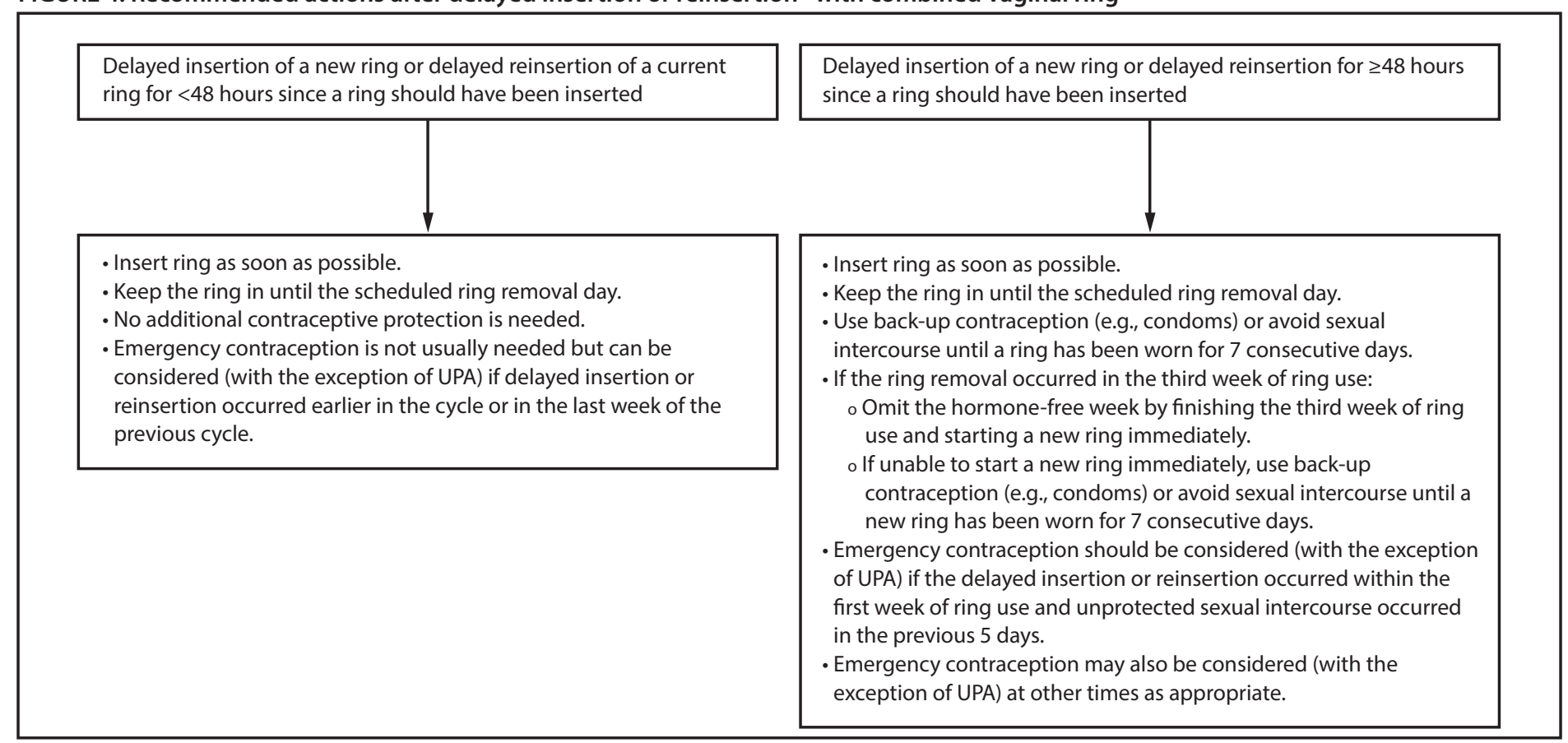

Abbreviation: UPA = ulipristal acetate.

* If removal takes place but the woman is unsure of how long the ring has been removed, consider the ring to have been removed for $\geq 48$ hours since a ring should have been inserted or reinserted.

ethinyl estradiol and progestin norelgestromin remained within reference ranges after extending patch wear for 3 days (266). No studies were found on extending the patch-free interval. In studies that provide indirect evidence on the effects of missed combined hormonal contraception on surrogate measures of pregnancy, how differences in surrogate measures correspond to pregnancy risk is unclear (Level of evidence: I, good, indirect to II-3, poor, direct).

\section{Vomiting or Severe Diarrhea While Using COCs}

Certain steps should be taken by women who experience vomiting or severe diarrhea while using COCs (Figure 5).

Comments and Evidence Summary. Theoretically, the contraceptive effectiveness of COCs might be decreased because of vomiting or severe diarrhea. Because of the lack of evidence that addresses vomiting or severe diarrhea while using COCs, these recommendations are based on the recommendations for missed COCs. No evidence was found on the effects of vomiting or diarrhea on measures of contraceptive effectiveness including pregnancy, follicular development, hormone levels, or cervical mucus quality.

\section{Unscheduled Bleeding with Extended or Continuous Use of Combined Hormonal Contraceptives}

- Before initiation of combined hormonal contraceptives, provide counseling about potential changes in bleeding patterns during extended or continuous combined hormonal contraceptive use. (Extended contraceptive use is defined as a planned hormone-free interval after at least two contiguous cycles. Continuous contraceptive use is defined as uninterrupted use of hormonal contraception without a hormone-free interval) (272).

- Unscheduled spotting or bleeding is common during the first 3-6 months of extended or continuous combined hormonal contraceptive use. It is generally not harmful and decreases with continued combined hormonal contraceptive use.

- If clinically indicated, consider an underlying gynecological problem, such as inconsistent use, interactions with other medications, cigarette smoking, an STD, pregnancy, or new pathologic uterine conditions (e.g., polyps or fibroids). If an underlying gynecological problem is found, treat the condition or refer for care.

- If an underlying gynecological problem is not found and the woman wants treatment, the following treatment option can be considered: 
FIGURE 5. Recommended actions after vomiting or diarrhea while using combined oral contraceptives

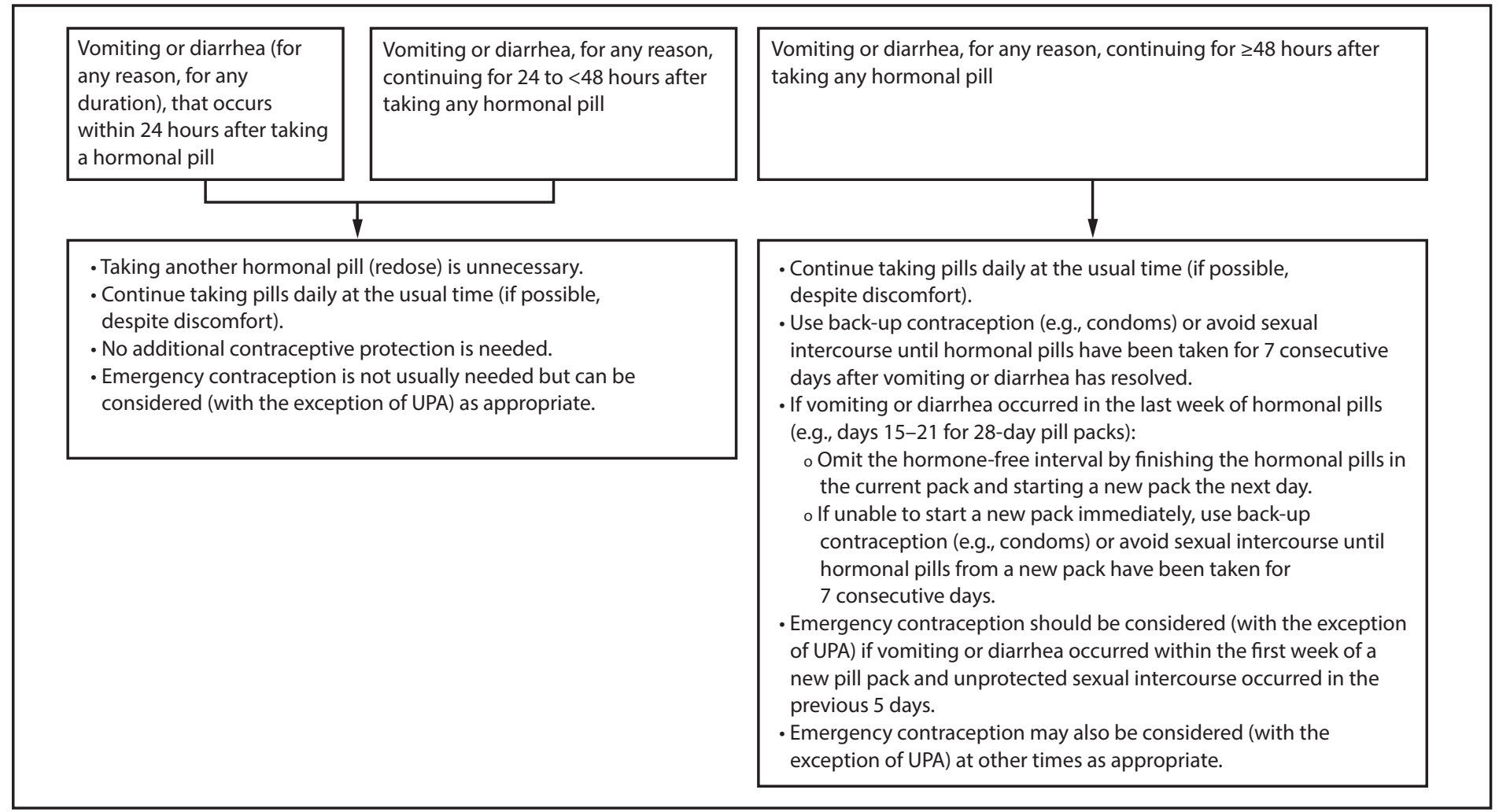

Abbreviation: UPA = ulipristal acetate.

- Advise the woman to discontinue combined hormonal contraceptive use (i.e., a hormone-free interval) for 3-4 consecutive days; a hormone-free interval is not recommended during the first 21 days of using the continuous or extended combined hormonal contraceptive method. A hormone-free interval also is not recommended more than once per month because contraceptive effectiveness might be reduced.

- If unscheduled spotting or bleeding persists and the woman finds it unacceptable, counsel her on alternative contraceptive methods, and offer another method if it is desired.

Comments and Evidence Summary. During contraceptive counseling and before initiating extended or continuous combined hormonal contraceptives, information about common side effects such as unscheduled spotting or bleeding, especially during the first 3-6 months of use, should be discussed (273). These bleeding irregularities are generally not harmful and usually improve with persistent use of the hormonal method. To avoid unscheduled spotting or bleeding, counseling should emphasize the importance of correct use and timing; for users of contraceptive pills, emphasize consistent pill use. Enhanced counseling about expected bleeding patterns and reassurance that bleeding irregularities are generally not harmful has been shown to reduce method discontinuation in clinical trials with DMPA $(124,125,274)$.

A systematic review identified three studies with small study populations that addressed treatments for unscheduled bleeding among women using extended or continuous combined hormonal contraceptives (275). In two separate randomized clinical trials in which women were taking either contraceptive pills or using the contraceptive ring continuously for 168 days, women assigned to a hormone-free interval of 3 or 4 days reported improved bleeding. Although they noted an initial increase in flow, this was followed by an abrupt decrease 7-8 days later with eventual cessation of flow 11-12 days later. These findings were compared with women who continued to use their method without a hormonefree interval, in which a greater proportion reported either treatment failure or fewer days of amenorrhea $(276,277)$. In another randomized trial of 66 women with unscheduled bleeding among women using 84 days of hormonally active contraceptive pills, oral doxycycline (100 mg twice daily) initiated the first day of bleeding and taken for 5 days did not result in any improvement in bleeding compared with placebo (278) (Level of evidence: I, fair, direct). 


\section{Progestin-Only Pills}

POPs contain only a progestin and no estrogen and are available in the United States. Approximately 9 out of 100 women become pregnant in the first year of use with POPs with typical use (14). POPs are reversible and can be used by women of all ages. POPs do not protect against STDs; consistent and correct use of male latex condoms reduces the risk for STDs, including HIV.

\section{Initiation of POPs}

\section{Timing}

- POPs can be started at any time if it is reasonably certain that the woman is not pregnant (Box 2).

\section{Need for Back-Up Contraception}

- If POPs are started within the first 5 days since menstrual bleeding started, no additional contraceptive protection is needed.

- If POPs are started $>5$ days since menstrual bleeding started, the woman needs to abstain from sexual intercourse or use additional contraceptive protection for the next 2 days.

\section{Special Considerations}

\section{Amenorrhea (Not Postpartum)}

- Timing: POPs can be started at any time if it is reasonably certain that the woman is not pregnant (Box 2).

- Need for back-up contraception: The woman needs to abstain from sexual intercourse or use additional contraceptive protection for the next 2 days.

\section{Postpartum (Breastfeeding)}

- Timing: POPs can be started at any time, including immediately postpartum (U.S. MEC 2 if $<1$ month postpartum; U.S. MEC 1 if $\geq 1$ month postpartum) if it is reasonably certain that the woman is not pregnant (Box 2).

- Need for back-up contraception: If the woman is $<6$ months postpartum, amenorrheic, and fully or nearly fully breastfeeding (exclusively breastfeeding or the vast majority [ $\geq 85 \%$ ] of feeds are breastfeeds) (27), no additional contraceptive protection is needed. Otherwise, a woman who is $\geq 21$ days postpartum and has not experienced return of her menstrual cycles, she needs to abstain from sexual intercourse or use additional contraceptive protection for the next 2 days. If her menstrual cycles have returned and it has been $>5$ days since menstrual bleeding started, she needs to abstain from sexual intercourse or use additional contraceptive protection for the next 2 days.

\section{Postpartum (Not Breastfeeding)}

- Timing: POPs can be started at any time, including immediately postpartum (U.S. MEC 1), if it is reasonably certain that the woman is not pregnant (Box 2).

- Need for back-up contraception: If a woman is $<21$ days postpartum, no additional contraceptive protection is needed. Women who are $\geq 21$ days postpartum and whose menstrual cycles have not returned need to abstain from sexual intercourse or use additional contraceptive protection for the next 2 days. If her menstrual cycles have returned and it has been $>5$ days since menstrual bleeding started, she needs to abstain from sexual intercourse or use additional contraceptive protection for the next 2 days.

\section{Postabortion (Spontaneous or Induced)}

- Timing: POPs can be started within the first 7 days, including immediately postabortion (U.S. MEC 1).

- Need for back-up contraception: The woman needs to abstain from sexual intercourse or use additional contraceptive protection for the next 2 days unless POPs are started at the time of a surgical abortion.

\section{Switching from Another Contraceptive Method}

- Timing: POPs can be started immediately if it is reasonably certain that the woman is not pregnant (Box 2). Waiting for her next menstrual cycle is unnecessary.

- Need for back-up contraception: If it has been $>5$ days since menstrual bleeding started, the woman needs to abstain from sexual intercourse or use additional contraceptive protection for the next 2 days.

- Switching from an IUD: If the woman has had sexual intercourse since the start of her current menstrual cycle and it has been $>5$ days since menstrual bleeding started, theoretically, residual sperm might be in the genital tract, which could lead to fertilization if ovulation occurs. A health care provider may consider any of the following options:

- Advise the women to retain the IUD for at least 2 days after POPs are initiated and return for IUD removal.

- Advise the woman to abstain from sexual intercourse or use barrier contraception for 7 days before removing the IUD and switching to the new method.

- If the woman cannot return for IUD removal and has not abstained from sexual intercourse or used barrier contraception for 7 days, advise the woman to use ECPs at the time of IUD removal. POPs can be started immediately after use of ECPs (with the exception of UPA). POPs can be started no sooner than 5 days after use of UPA.

Comments and Evidence Summary. In situations in which the health care provider is uncertain whether the woman might 
be pregnant, the benefits of starting POPs likely exceed any risk; therefore, starting POPs should be considered at any time, with a follow-up pregnancy test in 2-4 weeks.

Unlike COCs, POPs inhibit ovulation in about half of cycles, although the rates vary widely by individual (279). Peak serum steroid levels are reached about 2 hours after administration, followed by rapid distribution and elimination, such that by 24 hours after administration, serum steroid levels are near baseline (279). Therefore, taking POPs at approximately the same time each day is important. An estimated 48 hours of POP use has been deemed necessary to achieve the contraceptive effects on cervical mucus (279). If a woman needs to use additional contraceptive protection when switching to POPs from another contraceptive method, consider continuing her previous method for 2 days after starting POPs. No direct evidence was found regarding the effects of starting POPs at different times of the cycle.

\section{Examinations and Tests Needed Before Initiation of POPs}

Among healthy women, no examinations or tests are needed before initiation of POPs, although a baseline weight and BMI measurement might be useful for monitoring POP users over time (Table 5). Women with known medical problems or other special conditions might need additional examinations or tests before being determined to be appropriate candidates for a particular method of contraception. The U.S. MEC might be useful in such circumstances (5).

Comments and Evidence Summary. Weight (BMI): Obese women can use POPs (U.S. MEC 1) (5); therefore, screening for obesity is not necessary for the safe initiation of POPs. However, measuring weight and calculating BMI at baseline might be helpful for monitoring any changes and counseling women who might be concerned about weight change perceived to be associated with their contraceptive method.

Bimanual examination and cervical inspection: Pelvic examination is not necessary before initiation of POPs because it does not facilitate detection of conditions for which POPs would be unsafe. Women with current breast cancer should not use POPs (U.S. MEC 4), and women with certain liver diseases generally should not use POPs (U.S. MEC 3) (5); however, neither of these conditions are likely to be detected by pelvic examination (145). A systematic review identified two casecontrol studies that compared delayed versus immediate pelvic examination before initiation of hormonal contraceptives, specifically oral contraceptives or DMPA (95). No differences in risk factors for cervical neoplasia, incidence of STDs, incidence of abnormal Papanicolaou smears, or incidence of
TABLE 5. Classification of examinations and tests needed before progestin-only pill initiation

\begin{tabular}{ll}
\hline Examination or test & Class $^{*}$ \\
\hline Examination & \\
Blood pressure & $\mathrm{C}$ \\
Weight (BMI) (weight $[\mathrm{kg}] /$ height $[\mathrm{m}]^{2}{ }^{\text {) }}$ & \\
Clinical breast examination & $\mathrm{C}$ \\
Bimanual examination and cervical inspection & $\mathrm{C}$ \\
Laboratory test & \\
Glucose & $\mathrm{C}$ \\
Lipids & $\mathrm{C}$ \\
Liver enzymes & $\mathrm{C}$ \\
Hemoglobin & $\mathrm{C}$ \\
Thrombogenic mutations & $\mathrm{C}$ \\
Cervical cytology (Papanicolaou smear) & $\mathrm{C}$ \\
STD screening with laboratory tests & $\mathrm{C}$ \\
HIV screening with laboratory tests & $\mathrm{C}$ \\
\hline
\end{tabular}

Abbreviations: $\mathrm{BMI}=$ body mass index; $\mathrm{HIV}=$ human immunodeficiency virus; $\mathrm{STD}=$ sexually transmitted disease; U.S. MEC = U.S. Medical Eligibility Criteria for Contraceptive Use.

* Class A: essential and mandatory in all circumstances for safe and effective use of the contraceptive method. Class B: contributes substantially to safe and effective use, but implementation may be considered within the public health and/or service context; the risk of not performing an examination or test should be balanced against the benefits of making the contraceptive method available. Class C: does not contribute substantially to safe and effective use of the contraceptive method.

+ Weight (BMI) measurement is not needed to determine medical eligibility for any methods of contraception because all methods can be used (U.S. MEC 1) or generally can be used (U.S. MEC 2) among obese women (Box 1). However, measuring weight and calculating BMl at baseline might be helpful for monitoring any changes and counseling women who might be concerned about weight change perceived to be associated with their contraceptive method.

abnormal findings from wet mounts were observed (Level of evidence: II-2 fair, direct).

Lipids: Screening for dyslipidemias is not necessary for the safe initiation of POPs because of the low prevalence of undiagnosed disease in women of reproductive age and the low likelihood of clinically significant changes with use of hormonal contraceptives. A systematic review did not identify any evidence regarding outcomes among women who were screened versus not screened with lipid measurement before initiation of hormonal contraceptives (57). During 2009-2012 among women aged 20-44 years in the United States, 7.6\% had high cholesterol, defined as total serum cholesterol $\geq 240 \mathrm{mg} / \mathrm{dL}$ (84). During 1999-2008, the prevalence of undiagnosed hypercholesterolemia among women aged $20-44$ years was approximately $2 \%$ (85). Studies have shown mixed results about the effects of hormonal methods on lipid levels among both healthy women and women with baseline lipid abnormalities, and the clinical significance of these changes is unclear (86-89).

Liver enzymes: Although women with certain liver diseases generally should not use POPs (U.S. MEC 3) (5), screening for liver disease before initiation of POPs is not necessary because of the low prevalence of these conditions and the high likelihood that women with liver disease already would 
have had the condition diagnosed. A systematic review did not identify any evidence regarding outcomes among women who were screened versus not screened with liver enzyme tests before initiation of hormonal contraceptives (57). In 2012, among U.S. women, the percentage with liver disease (not further specified) was $1.3 \%(90)$. In 2013, the incidence of acute hepatitis A, B, or $\mathrm{C}$ was $\leq 1$ per 100,000 U.S. population (91). During 2002-2011, the incidence of liver carcinoma among U.S. women was approximately 3.7 per 100,000 population (92). Because estrogen and progestins are metabolized in the liver, the use of hormonal contraceptives among women with liver disease might, theoretically, be a concern. The use of hormonal contraceptives, specifically COCs and POPs, does not affect disease progression or severity in women with hepatitis, cirrhosis, or benign focal nodular hyperplasia $(93,94)$.

Clinical breast examination: Although women with current breast cancer should not use POPs (U.S. MEC 4) (5), screening asymptomatic women with a clinical breast examination before initiating POPs is not necessary because of the low prevalence of breast cancer among women of reproductive age. A systematic review did not identify any evidence regarding outcomes among women who were screened versus not screened with a clinical breast examination before initiation of hormonal contraceptives (95). The incidence of breast cancer among women of reproductive age in the United States is low. In 2012, the incidence of breast cancer among women aged 20-49 years was approximately 70.7 per 100,000 women (96).

Other screening: Women with hypertension, diabetes, anemia, thrombogenic mutations, cervical intraepithelial neoplasia, cervical cancer, STDs, or HIV infection can use (U.S. MEC 1) or generally can use (U.S. MEC 2) POPs (5); therefore, screening for these conditions is not necessary for the safe initiation of POPs.

\section{Number of Pill Packs that Should Be Provided at Initial and Return Visits}

- At the initial and return visit, provide or prescribe up to a 1-year supply of POPs (e.g., 13 28-day pill packs), depending on the woman's preferences and anticipated use.

- A woman should be able to obtain POPs easily in the amount and at the time she needs them.

Comments and Evidence Summary. The more pill packs given up to 13 cycles, the higher the continuation rates. Restricting the number of pill packs distributed or prescribed can result in unwanted discontinuation of the method and increased risk for pregnancy.

A systematic review of the evidence suggested that providing a greater number of pill packs was associated with increased continuation (232). Studies that compared provision of one versus 12 packs, one versus 12 or 13 packs, or three versus seven packs found increased continuation of pill use among women provided with more pill packs (233-235). However, one study found no difference in continuation when patients were provided one and then three packs versus four packs all at once (236). In addition to continuation, a greater number of pill packs provided was associated with fewer pregnancy tests, fewer pregnancies, and lower cost per client. However, a greater number of pill packs (13 packs versus three packs) also was associated with increased pill wastage in one study (234) (Level of evidence: I to II-2, fair, direct).

\section{Routine Follow-Up After POP Initiation}

These recommendations address when routine follow-up is recommended for safe and effective continued use of contraception for healthy women. The recommendations refer to general situations and might vary for different users and different situations. Specific populations who might benefit from more frequent follow-up visits include adolescents, those with certain medical conditions or characteristics, and those with multiple medical conditions.

- Advise the woman to return at any time to discuss side effects or other problems or if she wants to change the method being used. No routine follow-up visit is required.

- At other routine visits, health care providers seeing POP users should do the following:

- Assess the woman's satisfaction with her contraceptive method and whether she has any concerns about method use.

- Assess any changes in health status, including medications, that would change the appropriateness of POPs for safe and effective continued use based on U.S. MEC (e.g., category 3 and 4 conditions and characteristics).

- Consider assessing weight changes and counseling women who are concerned about weight change perceived to be associated with their contraceptive method.

Comments and Evidence Summary. No evidence was found regarding whether a routine follow-up visit after initiating POPs improves correct and continued use.

\section{Missed POPs}

For the following recommendations, a dose is considered missed if it has been $>3$ hours since it should have been taken.

- Take one pill as soon as possible.

- Continue taking pills daily, one each day, at the same time each day, even if it means taking two pills on the same day.

- Use back-up contraception (e.g., condoms) or avoid sexual intercourse until pills have been taken correctly, on time, for 2 consecutive days. 
- Emergency contraception should be considered (with the exception of UPA) if the woman has had unprotected sexual intercourse.

Comments and Evidence Summary. Inconsistent or incorrect use of oral contraceptive pills is a major reason for oral contraceptive failure. Unlike COCs, POPs inhibit ovulation in about half of cycles, although this rate varies widely by individual (279). Peak serum steroid levels are reached about 2 hours after administration, followed by rapid distribution and elimination, such that by 24 hours after administration, serum steroid levels are near baseline (279). Therefore, taking POPs at approximately the same time each day is important. An estimated 48 hours of POP use was deemed necessary to achieve the contraceptive effects on cervical mucus (279). Women who frequently miss POPs should consider an alternative contraceptive method that is less dependent on the user to be effective (e.g., IUD, implant, or injectable). No evidence was found regarding the effects of missed POPs available in the United States on measures of contraceptive effectiveness including pregnancy, follicular development, hormone levels, or cervical mucus quality.

\section{Vomiting or Diarrhea (for any Reason or Duration) that Occurs Within 3 Hours After Taking a Pill}

- Take another pill as soon as possible (if possible, despite discomfort).

- Continue taking pills daily, one each day, at the same time each day.

- Use back-up contraception (e.g., condoms) or avoid sexual intercourse until 2 days after vomiting or diarrhea has resolved.

- Emergency contraception should be considered (with the exception of UPA) if the woman has had unprotected sexual intercourse.

Comments and Evidence Summary. Theoretically, the contraceptive effectiveness of POPs might be decreased because of vomiting or severe diarrhea. Because of the lack of evidence to address this question, these recommendations are based on the recommendations for missed POPs. No evidence was found regarding the effects of vomiting or diarrhea on measures of contraceptive effectiveness, including pregnancy, follicular development, hormone levels, or cervical mucus quality.

\section{Standard Days Method}

SDM is a method based on fertility awareness; users must avoid unprotected sexual intercourse on days 8-19 of the menstrual cycle (280). Approximately 5 out of 100 women become pregnant in the first year of use with perfect (i.e., correct and consistent) use of SDM (280); effectiveness based on typical use is not available for this method but is expected to be lower than that for perfect use. SDM is reversible and can be used by women of all ages. SDM does not protect against STDs; consistent and correct use of male latex condoms reduces the risk for STDs, including HIV.

\section{Use of SDM Among Women with Various Durations of the Menstrual Cycle}

\section{Menstrual Cycles of 26-32 Days}

- The woman may use the method.

- Provide a barrier method of contraception for protection on days 8-19 if she wants one.

- If she has unprotected sexual intercourse during days 8-19, consider the use of emergency contraception if appropriate.

\section{Two or More Cycles of $<26$ or $>32$ Days Within Any} 1 Year of SDM Use

- Advise the woman that the method might not be appropriate for her because of a higher risk for pregnancy. Help her consider another method.

Comments and Evidence Summary. The probability of pregnancy is increased when the menstrual cycle is outside the range of 26-32 days, even if unprotected sexual intercourse is avoided on days 8-19. A study of 7,600 menstrual cycles, including information on cycle length and signs of ovulation, concluded that the theoretical effectiveness of SDM is greatest for women with cycles of 26-32 days, that the method is still effective for women who occasionally have a cycle outside this range, and that it is less effective for women who consistently have cycles outside this range. Information from daily hormonal measurements shows that the timing of the 6-day fertile window varies greatly, even among women with regular cycles $(21,281,282)$.

\section{Emergency Contraception}

Emergency contraception consists of methods that can be used by women after sexual intercourse to prevent pregnancy. Emergency contraception methods have varying ranges of effectiveness depending on the method and timing of administration. Four options are available in the United States: the $\mathrm{Cu}-\mathrm{IUD}$ and three types of ECPs. 


\section{Types of Emergency Contraception}

\section{Intrauterine Device}

- $\mathrm{Cu}-\mathrm{IUD}$

\section{ECPs}

- UPA in a single dose (30 mg)

- Levonorgestrel in a single dose $(1.5 \mathrm{mg})$ or as a split dose (1 dose of $0.75 \mathrm{mg}$ of levonorgestrel followed by a second dose of $0.75 \mathrm{mg}$ of levonorgestrel 12 hours later)

- Combined estrogen and progestin in 2 doses (Yuzpe regimen: 1 dose of $100 \mu \mathrm{g}$ of ethinyl estradiol plus $0.50 \mathrm{mg}$ of levonorgestrel followed by a second dose of $100 \mu \mathrm{g}$ of ethinyl estradiol plus $0.50 \mathrm{mg}$ of levonorgestrel 12 hours later)

\section{Initiation of Emergency Contraception}

\section{Timing}

\section{Cu-IUD}

- The Cu-IUD can be inserted within 5 days of the first act of unprotected sexual intercourse as an emergency contraceptive.

- In addition, when the day of ovulation can be estimated, the $\mathrm{Cu}-\mathrm{IUD}$ can be inserted beyond 5 days after sexual intercourse, as long as insertion does not occur $>5$ days after ovulation.

\section{ECPs}

- ECPs should be taken as soon as possible within 5 days of unprotected sexual intercourse.

Comments and Evidence Summary. Cu-IUDs are highly effective as emergency contraception (283) and can be continued as regular contraception. UPA and levonorgestrel ECPs have similar effectiveness when taken within 3 days after unprotected sexual intercourse; however, UPA has been shown to be more effective than the levonorgestrel formulation 3-5 days after unprotected sexual intercourse (284). The combined estrogen and progestin regimen is less effective than UPA or levonorgestrel and also is associated with more frequent occurrence of side effects (nausea and vomiting) (285). The levonorgestrel formulation might be less effective than UPA among obese women (286).

Two studies of UPA use found consistent decreases in pregnancy rates when administered within 120 hours of unprotected sexual intercourse $(284,287)$. Five studies found that the levonorgestrel and combined regimens decreased risk for pregnancy through the fifth day after unprotected sexual intercourse; however, rates of pregnancy were slightly higher when ECPs were taken after 3 days (288-292). A meta-analysis of levonorgestrel ECPs found that pregnancy rates were low when administered within 4 days after unprotected sexual intercourse but increased at 4-5 days (293) (Level of evidence: I to II-2, good to poor, direct).

\section{Advance Provision of ECPs}

- An advance supply of ECPs may be provided so that ECPs will be available when needed and can be taken as soon as possible after unprotected sexual intercourse.

Comments and Evidence Summary. A systematic review identified 17 studies that reported on safety or effectiveness of advance ECPs in adult or adolescent women (294). Any use of ECPs was two to seven times greater among women who received an advance supply of ECPs. However, a summary estimate (relative risk $=0.97 ; 95 \%$ confidence interval $=0.77-1.22$ ) of five randomized controlled trials did not indicate a significant reduction in unintended pregnancies at 12 months with advance provision of ECPs. In the majority of studies among adults or adolescents, patterns of regular contraceptive use, pregnancy rates, and incidence of STDs did not vary between those who received advance ECPs and those who did not. Although available evidence supports the safety of advance provision of ECPs, effectiveness of advance provision of ECPs in reducing pregnancy rates at the population level has not been demonstrated (Level of evidence: I to II-3, good to poor, direct).

\section{Initiation of Regular Contraception After ECPs}

\section{UPA}

- Advise the woman to start or resume hormonal contraception no sooner than 5 days after use of UPA, and provide or prescribe the regular contraceptive method as needed. For methods requiring a visit to a health care provider, such as DMPA, implants, and IUDs, starting the method at the time of UPA use may be considered; the risk that the regular contraceptive method might decrease the effectiveness of UPA must be weighed against the risk of not starting a regular hormonal contraceptive method.

- The woman needs to abstain from sexual intercourse or use barrier contraception for the next 7 days after starting or resuming regular contraception or until her next menses, whichever comes first.

- Any nonhormonal contraceptive method can be started immediately after the use of UPA.

- Advise the woman to have a pregnancy test if she does not have a withdrawal bleed within 3 weeks. 


\section{Levonorgestrel and Combined Estrogen and Progestin ECPs}

- Any regular contraceptive method can be started immediately after the use of levonorgestrel or combined estrogen and progestin ECPs.

- The woman needs to abstain from sexual intercourse or use barrier contraception for 7 days.

- Advise the woman to have a pregnancy test if she does not have a withdrawal bleed within 3 weeks.

Comments and Evidence Summary. The resumption or initiation of regular hormonal contraception after ECP use involves consideration of the risk for pregnancy if ECPs fail and the risks for unintended pregnancy if contraception initiation is delayed until the subsequent menstrual cycle. A health care provider may provide or prescribe pills, the patch, or the ring for a woman to start no sooner than 5 days after use of UPA. For methods requiring a visit to a health care provider, such as DMPA, implants, and IUDs, starting the method at the time of UPA use may be considered; the risk that the regular contraceptive method might decrease the effectiveness of UPA must be weighed against the risk of not starting a regular hormonal contraceptive method.

Data on when a woman can start regular contraception after ECPs are limited to pharmacodynamic data and expert opinion (295-297). In one pharmacodynamic study of women who were randomly assigned to either UPA or placebo groups mid-cycle followed by a 21-day course of combined hormonal contraception found no difference between UPA and placebo groups in the time for women's ovaries to reach quiescence by ultrasound and serum estradiol (296); this finding suggests that UPA did not have an effect on the combined hormonal contraception. In another pharmacodynamic study with a crossover design, women were randomly assigned to one of three groups: 1) UPA followed by desogestrel for 20 days started 1 day later; 2) UPA plus placebo; or 3) placebo plus desogestrel for 20 days (295). Among women taking UPA followed by desogestrel, a higher incidence of ovulation in the first 5 days was found compared with UPA alone (45\% versus $3 \%$, respectively), suggesting desogestrel might decrease the effectiveness of UPA. No concern exists that administering combined estrogen and progestin or levonorgestrel formulations of ECPs concurrently with systemic hormonal contraception decreases the effectiveness of either emergency or regular contraceptive methods because these formulations do not have antiprogestin properties like UPA. If a woman is planning to initiate contraception after the next menstrual bleeding after ECP use, the cycle in which ECPs are used might be shortened, prolonged, or involve unscheduled bleeding.

\section{Prevention and Management of Nausea and Vomiting with ECP Use}

\section{Nausea and Vomiting}

- Levonorgestrel and UPA ECPs cause less nausea and vomiting than combined estrogen and progestin ECPs.

- Routine use of antiemetics before taking ECPs is not recommended. Pretreatment with antiemetics may be considered depending on availability and clinical judgment.

\section{Vomiting Within 3 Hours of Taking ECPs}

- Another dose of ECP should be taken as soon as possible. Use of an antiemetic should be considered.

Comments and Evidence Summary. Many women do not experience nausea or vomiting when taking ECPs, and predicting which women will experience nausea or vomiting is difficult. Although routine use of antiemetics before taking ECPs is not recommended, antiemetics are effective in some women and can be offered when appropriate. Health care providers who are deciding whether to offer antiemetics to women taking ECPs should consider the following: 1) women taking combined estrogen and progestin ECPs are more likely to experience nausea and vomiting than those who take levonorgestrel or UPA ECPs; 2) evidence indicates that antiemetics reduce the occurrence of nausea and vomiting in women taking combined estrogen and progestin ECPs; and 3) women who take antiemetics might experience other side effects from the antiemetics.

A systematic review examined incidence of nausea and vomiting with different ECP regimens and effectiveness of antinausea drugs in reducing nausea and vomiting with ECP use (298). The levonorgestrel regimen was associated with significantly less nausea than a nonstandard dose of UPA $(50 \mathrm{mg})$ and the standard combined estrogen and progestin regimen (299-301). Use of the split-dose levonorgestrel showed no differences in nausea and vomiting compared with the single-dose levonorgestrel $(288,290,292,302)$ (Level of evidence: I, good-fair, indirect). Two trials of antinausea drugs, meclizine and metoclopramide, taken before combined estrogen and progestin ECPs, reduced the severity of nausea $(303,304)$. Significantly less vomiting occurred with meclizine but not metoclopramide (Level of evidence: I, good-fair, direct). No direct evidence was found regarding the effects of vomiting after taking ECPs.

\section{Female Sterilization}

Laparoscopic, abdominal, and hysteroscopic methods of female sterilization are available in the United States, and 
some of these procedures can be performed in an outpatient procedure or office setting. Fewer than 1 out of 100 women become pregnant in the first year after female sterilization (14). Because these methods are intended to be irreversible, all women should be appropriately counseled about the permanency of sterilization and the availability of highly effective, long-acting, reversible methods of contraception. Female sterilization does not protect against STDs; consistent and correct use of male latex condoms reduces the risk for STDs, including HIV.

\section{When Hysteroscopic Sterilization is Reliable for Contraception}

- Before a woman can rely on hysteroscopic sterilization for contraception, a hysterosalpingogram (HSG) must be performed 3 months after the sterilization procedure to confirm bilateral tubal occlusion.

- The woman should be advised that she needs to abstain from sexual intercourse or use additional contraceptive protection until she has confirmed bilateral tubal occlusion.

\section{When Laparoscopic and Abdominal Approaches are Reliable for Contraception}

- A woman can rely on sterilization for contraception immediately after laparoscopic and abdominal approaches. No additional contraceptive protection is needed.

Comments and Evidence Summary. HSG confirmation is necessary to confirm bilateral tubal occlusion after hysteroscopic sterilization. The inserts for the hysteroscopic sterilization system available in the United States are placed bilaterally into the fallopian tubes and require 3 months for adequate fibrosis and scarring leading to bilateral tubal occlusion. After hysteroscopic sterilization, advise the woman to correctly and consistently use an effective method of contraception while awaiting confirmation. If compliance with another method might be a problem, a woman and her health care provider may consider DMPA injection at the time of sterilization to ensure adequate contraception for 3 months. Unlike laparoscopic and abdominal sterilizations, pregnancy risk beyond 7 years of follow-up has not been studied among women who received hysteroscopic sterilization.

Pregnancy risk with at least 10 years of follow-up has been studied among women who received laparoscopic and abdominal sterilizations (305,306). Although these methods are highly effective, pregnancies can occur many years after the procedure, and the risk for pregnancy is higher among younger women $(306,307)$.
A systematic review was conducted to identify studies that reported whether pregnancies occurred after hysteroscopic sterilization (308). Twenty-four studies were identified that reported whether pregnancies occurred after hysteroscopic sterilization and found that very few pregnancies occurred among women with confirmed bilateral tubal occlusion; however, few studies include long-term follow-up, and none with follow up for $>7$ years. Among women who had successful bilateral placement, most pregnancies that occurred after hysteroscopic sterilization were in women who did not have confirmed bilateral tubal occlusion at 3 months, either because of lack of follow up or misinterpretation of HSG results (309-311). Some pregnancies occurred within 3 months of placement, including among women who were already pregnant at the time of the procedure, women who did not use alternative contraception, or women who had failures of alternative contraception (310-315). Although these studies generally demonstrated high rates of bilateral placement, some pregnancies occurred as a result of lack of bilateral placement identified on later imaging $(310,311,313-316)$. Most pregnancies occurred after deviations from FDA directions, which include placement in the early follicular phase of the menstrual cycle, imaging at 3 months to document proper placement, and use of effective alternative contraception until documented occlusion (Level of evidence: II-3, fair, direct).

\section{Male Sterilization}

Male sterilization, or vasectomy, is one of the few contraceptive methods available to men and can be performed in an outpatient procedure or office setting. Fewer than 1 woman out of 100 becomes pregnant in the first year after her male partner undergoes sterilization (14). Because male sterilization is intended to be irreversible, all men should be appropriately counseled about the permanency of sterilization and the availability of highly effective, long-acting, reversible methods of contraception for women. Male sterilization does not protect against STDs; consistent and correct use of male latex condoms reduces the risk for STDs, including HIV.

\section{When Vasectomy is Reliable for Contraception}

- A semen analysis should be performed 8-16 weeks after a vasectomy to ensure the procedure was successful.

- The man should be advised that he should use additional contraceptive protection or abstain from sexual intercourse until he has confirmation of vasectomy success by postvasectomy semen analysis. 


\section{Other Postprocedure Recommendations}

- The man should refrain from ejaculation for approximately 1 week after the vasectomy to allow for healing of surgical sites and, after certain methods of vasectomy, occlusion of the vas.

Comments and Evidence Summary. The Vasectomy Guideline Panel of the American Urological Association performed a systematic review of key issues concerning the practice of vasectomy (317). All English-language publications on vasectomy published during 1949-2011 were reviewed. For more information, see the American Urological Association Vasectomy Guidelines (https://www.auanet.org/common/pdf/ education/clinical-guidance/Vasectomy.pdf).

Motile sperm disappear within a few weeks after vasectomy (318-321). The time to azoospermia varies widely in different studies; however, by 12 weeks after the vasectomy, $80 \%$ of men have azoospermia, and almost all others have rare nonmotile sperm (defined as $\leq 100,000$ nonmotile sperm per milliliter) (317). The number of ejaculations after vasectomy is not a reliable indicator of when azoospermia or rare nonmotile sperm will be achieved (317). Once azoospermia or rare nonmotile sperm has been achieved, patients can rely on the vasectomy for contraception, although not with $100 \%$ certainty. The risk for pregnancy after a man has achieved postvasectomy azoospermia is approximately one in 2,000 (322-326).

A median of $78 \%$ (range $33 \%-100 \%$ ) of men return for a single postvasectomy semen analysis (317). In the largest cohorts that appear typical of North American vasectomy practice, approximately two thirds of men $(55 \%-71 \%)$ return for at least one postvasectomy semen analysis (322,327-331). Assigning men an appointment after their vasectomy might improve compliance with follow-up (332).

\section{When Women Can Stop Using Contraceptives}

- Contraceptive protection is still needed for women aged $>44$ years if the woman wants to avoid pregnancy.

Comments and Evidence Summary. The age at which a woman is no longer at risk for pregnancy is not known. Although uncommon, spontaneous pregnancies occur among women aged $>44$ years. Both the American College of Obstetricians and Gynecologists and the North American Menopause Society recommend that women continue contraceptive use until menopause or age $50-55$ years $(333,334)$. The median age of menopause is approximately 51 years in North America (333) but can vary from ages 40-60 years (335). The median age of definitive loss of natural fertility is 41 years but can range up to age 51 years $(336,337)$. No reliable laboratory tests are available to confirm definitive loss of fertility in a woman. The assessment of follicle-stimulating hormone levels to determine when a woman is no longer fertile might not be accurate (333).

Health care providers should consider the risks for becoming pregnant in a woman of advanced reproductive age, as well as any risks of continuing contraception until menopause. Pregnancies among women of advanced reproductive age are at higher risk for maternal complications, such as hemorrhage, venous thromboembolism, and death, and fetal complications, such as spontaneous abortion, stillbirth, and congenital anomalies (338-340). Risks associated with continuing contraception, in particular risks for acute cardiovascular events (venous thromboembolism, myocardial infarction, or stroke) or breast cancer, also are important to consider. U.S. MEC states that on the basis of age alone, women aged $>45$ years can use POPs, implants, the LNG-IUD, or the Cu- IUD (U.S. MEC 1) (5). Women aged $>45$ years generally can use combined hormonal contraceptives and DMPA (U.S. MEC 2) (5). However, women in this age group might have chronic conditions or other risk factors that might render use of hormonal contraceptive methods unsafe; U.S. MEC might be helpful in guiding the safe use of contraceptives in these women.

In two studies, the incidence of venous thromboembolism was higher among oral contraceptive users aged $\geq 45$ years compared with younger oral contraceptive users (341-343); however, an interaction between hormonal contraception and increased age compared with baseline risk was not demonstrated $(341,342)$ or was not examined (343). The relative risk for myocardial infarction was higher among all oral contraceptive users than in nonusers, although a trend of increased relative risk with increasing age was not demonstrated $(344,345)$. No studies were found regarding the risk for stroke in COC users aged $\geq 45$ years (Level of evidence: II-2, good to poor, direct).

A pooled analysis by the Collaborative Group on Hormonal Factors and Breast Cancer in 1996 (346) found small increased relative risks for breast cancer among women aged $\geq 45$ years whose last use of combined hormonal contraceptives was $<5$ years previously and for those whose last use was 5-9 years previously. Seven more recent studies suggested small but nonsignificant increased relative risks for breast carcinoma in situ or breast cancer among women who had used oral contraceptives or DMPA when they were aged $\geq 40$ years compared with those who had never used either method (347-353) (Level of evidence: II-2, fair, direct). 


\section{Conclusion}

Most women can start most contraceptive methods at any time, and few examinations or tests, if any, are needed before starting a contraceptive method. Routine follow-up for most women includes assessment of her satisfaction with the contraceptive method, concerns about method use, and changes in health status or medications that could affect medical eligibility for continued use of the method. Because changes in bleeding patterns are one of the major reasons for discontinuation of contraception, recommendations are provided for the management of bleeding irregularities with various contraceptive methods. In addition, because women and health care providers can be confused about the procedures for missed pills and dosing errors with the contraceptive patch and ring, the instructions are streamlined for easier use. ECPs and emergency use of the $\mathrm{Cu}$-IUD are important options for women, and recommendations on using these methods, as well as starting regular contraception after use of emergency contraception, are provided. Male and female sterilization are highly effective methods of contraception for men, women, and couples who have completed childbearing; for men undergoing vasectomy and women undergoing a hysteroscopic sterilization procedure, additional contraceptive protection is needed until the success of the procedure can be confirmed.

$\mathrm{CDC}$ is committed to working with partners at the federal, national, and local levels to disseminate, implement, and evaluate U.S. SPR recommendations so that the information reaches health care providers. Strategies for dissemination and implementation include collaborating with other federal agencies and professional and service organizations to widely distribute the recommendations through presentations, electronic distribution, newsletters, and other publications; development of provider tools and job aids to assist providers in implementing the new recommendations; and training activities for students, as well as for continuing education. CDC conducts surveys of family planning health care providers to assess attitudes and practices related to contraceptive use. Results from these surveys will assist CDC in evaluating the impact of these recommendations on the provision of contraceptives in the United States. Finally, CDC will continually monitor new scientific evidence and will update these recommendations as warranted by new evidence. Updates to the recommendations, as well as provider tools and other resources, are available on the CDC U.S. SPR website: http:// www.cdc.gov/reproductivehealth/UnintendedPregnancy/ USSPR.htm.

\section{Acknowledgments}

This report is based, in part, on the work of the Promoting Family Planning Team, Department of Reproductive Health and Research, World Health Organization, and its development of the Selected Practice Recommendations for Contraceptive Use, 3rd edition.

\section{U.S. Selected Practice Recommendations for Contraceptive Use Participants}

CDC Guideline Development Group for U.S. Medical Eligibility Criteria for Contraceptive Use and U.S. Selected Practice Recommendations for Contraceptive Use

Kathryn M. Curtis, PhD (Chair), Erin Berry-Bibee, MD, Suzanne G. Folger, PhD, Leah G. Horton, MSPH, Denise J. Jamieson, MD, Tara C. Jatlaoui, MD, Polly A. Marchbanks, PhD, H. Pamela Pagano, MPH, Halley E.M. Riley, MPH, Mirelys Rodriguez, Katharine B. Simmons, MD, Naomi K. Tepper, MD, Maura K. Whiteman, PhD, Lauren B. Zapata, PhD, CDC, Atlanta, Georgia.

\section{Invited Meeting Participants}

August 27-28, 2014, Atlanta, Georgia

Herbert Peterson, MD, University of North Carolina, Chapel Hill, North Carolina (Chair); Gale Burstein, MD, Erie County Department of Health, Buffalo, New York; Alison Edelman, MD, Oregon Health and Science University, Portland, Oregon; Eve Espey, MD, University of New Mexico, Albuquerque, New Mexico; Emily Godfrey, MD, University of Washington, Seattle, WA; Andrew Kaunitz, MD, University of Florida, Jacksonville, Florida; Susan Moskosky, MS, U.S. Department of Health and Human Services, Rockville, Maryland; Kavita Nanda, MD, FHI360 and American College of Obstetricians and Gynecologists, Durham, NC; Deborah Nucatola, MD, Planned Parenthood Federation of America, New York, New York; Michael Policar, MD, University of California, San Francisco, California; Carolyn Westhoff, MD, Columbia University, New York, New York.

\section{Systematic Review Presenters and Authors \\ Meeting, August 26-28, 2015, Atlanta, Georgia}

Kathryn M. Curtis, PhD, Tara C. Jatlaoui, MD, Polly A. Marchbanks, PhD, Lauren B. Zapata, PhD, CDC, Atlanta, Georgia; Nathalie Kapp, MD, HRA Pharma, Paris, France. 


\section{Invited Meeting Participants}

\section{August 26-28, 2015, Atlanta, Georgia}

Herbert Peterson, MD, University of North Carolina, Chapel Hill, North Carolina (Chair); Rebecca Allen, MD, American Society for Reproductive Medicine and Women and Infants Hospital, Providence, Rhode Island; Abbey Berenson, MD, University of Texas Medical Branch, Galveston, Texas; Amanda Black, MD, University of Ottawa, Ontario, Canada; Cora Collette Breuner, MD, American Academy of Pediatrics and Seattle Children's Hospital, Seattle, Washington; Gale Burstein, MD, Erie County Department of Health, Buffalo, New York; Alicia Christy, MD, National Institutes of Health, Rockville, Maryland; Mitchell D. Creinin, MD, University of California, Davis, California; Linda Dominguez, Southwest Women's Health, Albuquerque, New Mexico; Alison Edelman, MD, Oregon Health and Science University, Portland, Oregon; Eve Espey, MD, University of New Mexico, Albuquerque, New Mexico; Emily Godfrey, MD, University of Washington, Seattle, WA; Marji Gold, MD, Albert Einstein College of Medicine, Bronx, New York; Robert Hatcher, MD, Emory University, Atlanta, Georgia; Mark Hathaway, MD, JHPIEGO and Unity Healthcare, Washington, DC; Stephen Heartwell, MD, Susan Thompson Buffett Foundation, Omaha, Nebraska; Paula Hillard, MD, Stanford University, Palo Alto, California; Andrew Kaunitz, MD, University of Florida, Jacksonville, Florida; Reagan McDonald-Mosley, MD, Planned Parenthood Federation of America, New York, New York; Susan Moskosky, MS, U.S. Department of Health and Human Services, Rockville, Maryland; Kavita Nanda, MD, FHI360, Durham, North Carolina; Jeffrey Peipert, MD, Washington University, St. Louis, Missouri; Michael Policar, MD, University of California, San Francisco, California; Sarah Prager, MD, University of Washington, Seattle Washington; David Soper, MD, Medical University of South Carolina, Charleston, South Carolina; Lisa Soule, MD, Food and Drug Administration, Rockville, Maryland; James Trussell, PhD, Princeton University, Princeton, New Jersey; Carolyn Westhoff, MD, Columbia University, New York, New York; Christopher Zahn, MD, American College of Obstetricians and Gynecologists, Washington, DC.

\section{CDC Attendees}

Wanda D. Barfield, MD, Peter Briss, MD, W. Craig Hooper, PhD, Jill Huppert, MD, Caroline King, PhD, Michele Mandel, Titilope Oduyebo, MD, Cria Perrine, MD, Sam Posner, PhD.

\section{External Reviewers}

Claude Burnett, MD, Northeast Health District, Georgia Department of Public Health, Athens, Georgia; Carrie Cwiak, MD, Emory University, Atlanta, Georgia; David Klein, MD, Uniformed Services University, Bethesda, Maryland; Seema Menon, MD, Medical College of Wisconsin, Milwaukee, Wisconsin.

\section{Conflicts of Interest for Invited Meeting Participants August 26-28, 2015, Atlanta, Georgia}

Rebecca Allen, Nexplanon trainer for Merck and Liletta trainer for Actavis, consultant, advisory board, and education grant from Bayer; Mitchell D. Creinin, Nexplanon trainer for Merck, litigation consultant for Bayer, advisory board for Merck and Teva Pharmaceutical Industries Ltd., consultant for Lemonaid — PolkaDoc app, research support to University of California, Davis from Medicines360, Contramed, Merck, Eunice Kennedy Shriver National Institute of Child Health and Human Development, and Society of Family Planning; Linda Dominguez, speaker for Bayer, Merck, and Actavis; Alison Edelman, royalties from Up to Date, Inc., consultant for Genzyme, grant support from National Institutes of Health and Gates Foundation, travel funds from World Health Organization, grant support and honorarium from Society of Family Planning, honorarium and travel funds from Contemporary Forum, trainer for Merck, consultant for Gynuity Health Projects, honorarium from CDC, Projects In Knowledge, and American Congress of Obstetricians and Gynecologists, advisory board for Agile Therapeutics; Eve Espey, travel funds from the American Congress of Obstetricians and Gynecologists, Society for Family Planning, and U.S. Food and Drug Administration, Reproductive and Drug Advisory Committee for U.S. Food and Drug Administration, travel funds and honoraria from Wayne State University, Telluride Conference, New Mexico Department of Health Clinician Conference, Planned Parenthood National Medical Conference and Society of Family Planning, British Columbia Contraception Access Research Team Conference, and American Congress of Obstetricians and Gynecologists annual meeting; Emily Godfrey, research funding from Bayer Women's Health, Prima-Temp, and Teva Pharmaceutical Industries Ltd., trainer for Merck and Upstream USA, grant reviewer for Fellowship of Family Planning and Society of Family Planning Research Fund; Mark Hathaway, Liletta trainer and speaker for Actavis and Medicines360, Nexplanon trainer for Merck, advisory board for Contramed LLC and Afaxys Pharmaceuticals; Paula Hillard consultant for American Civil Liberties Union, Advanced Health Media, CMEology, National Sleep Foundation, and Planned Parenthood Federation of America, honoraria from National Sleep Foundation, Dignity Health, CMEology, Advance Health Media, and Medscape, editorial board for Advanstar - Contemporary OB/GYN, board examiner for the American Board of Obstetrics and Gynecology, contract reviewer for the Department of Health and Human Services, editorial board for EBSCO — PEMSoft, Nexplanon trainer for Merck, scientific advisor to Proctor and Gamble, publication royalties from Wiley Blackwell Publishing; Nathalie Kapp, employee of HRA Pharma; Andrew Kaunitz, advisory board participant of Allergan, Bayer, Merck, and Pfizer, clinical trial funding to University of Florida from Agile Therapeutics, Bayer, Merck; Jeffrey Peipert, research funding from Bayer and Teva Pharmaceutical Industries Ltd., advisory board for Perrigo; Michael Policar, litigation consultant for Bayer; James Trussell, advisory board for Merck and Teva Pharmaceutical Industries Ltd., consultant for Bayer; Carolyn Westhoff, data and safety monitoring board for Merck and Bayer, advisory board for Agile Therapeutics, MicroChips Biotech, and Actavis, research support to Columbia University from Medicines360, León Farma, and ContraMed. 


\section{Handling Conflict of Interest}

To promote transparency, all participants were asked to disclose any potential conflicts of interest to CDC prior to the expert meeting and to report any potential conflicts of interest during the introductory portion of the expert meeting. All potential conflicts of interest are listed above. No participants were excluded from discussion based on potential conflicts of interest. One presenter was an employee of a pharmaceutical company and participated by teleconference; after the presentation and questions related to the presentation, the presenter was excused from the discussion. CDC staff who ultimately decided and developed these recommendations have no financial interests or other relationships with the manufacturers of commercial products, suppliers of commercial services, or commercial supporters relevant to these recommendations.

\section{References}

1. Finer LB, Zolna MR. Declines in unintended pregnancy in the United States, 2008-2011. N Engl J Med 2016;374:843-52. http://dx.doi. org/10.1056/NEJMsa1506575

2. Gipson JD, Koenig MA, Hindin MJ.The effects of unintended pregnancy on infant, child, and parental health: a review of the literature. Stud Fam Plann 2008;39:18-38. http://dx.doi.org/10.1111/j.1728-4465.2008.00148.x

3. Sonfield A, Kost K. Public costs from unintended pregnancies and the role of public insurance programs in paying for pregnancy-related care: national and state estimates for 2010. New York, NY: The Guttmacher Institute; 2015.

4. Finer LB, Henshaw SK. Disparities in rates of unintended pregnancy in the United States, 1994 and 2001. Perspect Sex Reprod Health 2006;38:90-6. http://dx.doi.org/10.1363/3809006

5. CDC. U.S. medical eligibility criteria for contraceptive use, 2016. MMWR Recomm Rep (No. RR-3);2016.

6. CDC. U.S. selected practice recommendations for contraceptive use, 2013: adapted from the World Health Organization selected practice recommendations for contraceptive use, 2nd edition. MMWR Recomm Rep 2013;62(No. RR-5).

7. Mohllajee AP, Curtis KM, Flanagan RG, Rinehart W, Gaffield ML, Peterson HB. Keeping up with evidence: a new system for WHO's evidence-based family planning guidance. Am J Prev Med 2005;28:48390. http://dx.doi.org/10.1016/j.amepre.2005.02.008

8. Liberati A, Altman DG, Tetzlaff J, et al. The PRISMA statement for reporting systematic reviews and meta-analyses of studies that evaluate health care interventions: explanation and elaboration. J Clin Epidemiol 2009;62:e1-34. http://dx.doi.org/10.1016/j.jclinepi.2009.06.006

9. Moher D, Liberati A, Tetzlaff J, Altman DG; PRISMA Group. Preferred reporting items for systematic reviews and meta-analyses: the PRISMA statement. BMJ 2009;339:b2535.

10. Harris RP, Helfand M, Woolf SH, et al; Methods Work Group, Third US Preventive Services Task Force. Current methods of the U.S. Preventive Services Task Force: a review of the process. Am J Prev Med 2001;20(Suppl):21-35. http://dx.doi.org/10.1016/S0749-3797(01)00261-6

11. Horton LG, Folger SG, Berry-Bibee E, Jatlaoui TC, Tepper NK, Curtis KM. Research gaps from evidence-based contraceptives guidance: the U.S. Medical Eligibility Criteria for Contraceptive Use, 2016, and the U.S. Selected Practice Recommendations for Contraceptive Use, 2016. Contraception. In press 2016.

12. Gavin L, Moskosky S, Carter M, et al. Providing quality family planning services: Recommendations of CDC and the U.S. Office of Population Affairs. MMWR Recomm Rep 2014;63(No. RR-4).

13. World Health Organization. Selected practice recommendations for contraceptive use. 2nd ed. Geneva, Switzerland: WHO Press; 2004.
14. Trussell J. Contraceptive failure in the United States. Contraception 2011;83:397-404. http://dx.doi.org/10.1016/j.contraception.2011.01.021

15. Workowski KA, Bolan GA. Sexually transmitted diseases treatment guidelines, 2015. MMWR Recomm Rep 2015;64(No. RR-3).

16. Stanback J, Nakintu N, Qureshi Z, Nasution M. Does assessment of signs and symptoms add to the predictive value of an algorithm to rule out pregnancy? J Fam Plann Reprod Health Care 2006;32:27-9. http:// dx.doi.org/10.1783/147118906775275370

17. Stanback J, Nanda K, Ramirez Y, Rountree W, Cameron SB. Validation of a job aid to rule out pregnancy among family planning clients in Nicaragua. Rev Panam Salud Publica 2008;23:116-8. http://dx.doi. org/10.1590/S1020-49892008000200007

18. Stanback J, Qureshi Z, Sekadde-Kigondu C, Gonzalez B, Nutley T. Checklist for ruling out pregnancy among family-planning clients in primary care. Lancet 1999;354:566. http://dx.doi.org/10.1016/ S0140-6736(99)01578-0

19. Torpey K, Mwenda L, Kabaso M, et al. Excluding pregnancy among women initiating antiretroviral therapy: efficacy of a family planning job aid. BMC Public Health 2010;10:249. http://dx.doi.org/10.1186/1471-2458-10-249

20. Cole LA, Ladner DG, Byrn FW. The normal variabilities of the menstrual cycle. Fertil Steril 2009;91:522-7. http://dx.doi.org/10.1016/j. fertnstert.2007.11.073

21. Wilcox AJ, Dunson D, Baird DD. The timing of the "fertile window" in the menstrual cycle: day specific estimates from a prospective study. BMJ 2000;321:1259-62. http://dx.doi.org/10.1136/bmj.321.7271.1259

22. Donnet ML, Howie PW, Marnie M, Cooper W, Lewis M. Return of ovarian function following spontaneous abortion. Clin Endocrinol (Oxf) 1990;33:13-20. http://dx.doi.org/10.1111/j.1365-2265.1990. tb00460.x

23. Lähteenmäki P. Postabortal contraception. Ann Med 1993;25:185-9. http://dx.doi.org/10.3109/07853899309164166

24. Stoddard A, Eisenberg DL. Controversies in family planning: timing of ovulation after abortion and the conundrum of postabortion intrauterine device insertion. Contraception 2011;84:119-21. http://dx.doi. org/10.1016/j.contraception.2010.12.010

25. Jackson E, Glasier A. Return of ovulation and menses in postpartum nonlactating women: a systematic review. Obstet Gynecol 2011;117:65762. http://dx.doi.org/10.1097/AOG.0b013e31820ce18c

26. Kennedy KI, Rivera R, McNeilly AS. Consensus statement on the use of breastfeeding as a family planning method. Contraception 1989;39:477-96. http://dx.doi.org/10.1016/0010-7824(89)90103-0

27. Labbok MH, Perez A, Valdes V, et al. The lactational amenorrhea method (LAM): a postpartum introductory family planning method with policy and program implications. Adv Contracept 1994;10:93-109. http:// dx.doi.org/10.1007/BF01978103

28. US Food and Drug Administration. $510(\mathrm{k})$ premarket notification. Silver Spring, MD; 2015. https://www.accessdata.fda.gov/scripts/cdrh/ cfdocs/cfpmn/pmn.cfm

29. Cervinski MA, Gronowski AM. Reproductive-endocrine point-of-care testing: current status and limitations. Clin Chem Lab Med 2010;48:935-42. http://dx.doi.org/10.1515/CCLM.2010.183

30. Cole LA. Human chorionic gonadotropin tests. Expert Rev Mol Diagn 2009;9:721-47. http://dx.doi.org/10.1586/erm.09.51

31. Eichner SF, Timpe EM. Urinary-based ovulation and pregnancy: pointof-care testing. Ann Pharmacother 2004;38:325-31. http://dx.doi. org/10.1345/aph.1D210

32. Wilcox AJ, Baird DD, Dunson D, McChesney R, Weinberg CR. Natural limits of pregnancy testing in relation to the expected menstrual period. JAMA 2001;286:1759-61. http://dx.doi.org/10.1001/jama.286.14.1759

33. Korhonen J, Alfthan H, Ylöstalo P, Veldhuis J, Stenman UH. Disappearance of human chorionic gonadotropin and its alpha- and beta-subunits after term pregnancy. Clin Chem 1997;43:2155-63. 
34. Reyes FI, Winter JS, Faiman C. Postpartum disappearance of chorionic gonadotropin from the maternal and neonatal circulations. Am J Obstet Gynecol 1985;153:486-9. http://dx.doi. org/10.1016/0002-9378(85)90458-2

35. Steier JA, Bergsjø P, Myking OL. Human chorionic gonadotropin in maternal plasma after induced abortion, spontaneous abortion, and removed ectopic pregnancy. Obstet Gynecol 1984;64:391-4.

36. Bracken MB. Oral contraception and congenital malformations in offspring: a review and meta-analysis of the prospective studies. Obstet Gynecol 1990;76:552-7.

37. Gray RH, Pardthaisong T. In utero exposure to steroid contraceptives and survival during infancy. Am J Epidemiol 1991;134:804-11.

38. Pardthaisong T, Gray RH. In utero exposure to steroid contraceptives and outcome of pregnancy. Am J Epidemiol 1991;134:795-803.

39. Jaffe B, Harlap S, Baras M, et al. Long-term effects of MPA on human progeny: intellectual development. Contraception 1988;37:607-19. http://dx.doi.org/10.1016/0010-7824(88)90007-8

40. Pardthaisong T, Yenchit C, Gray R. The long-term growth and development of children exposed to Depo-Provera during pregnancy or lactation. Contraception 1992;45:313-24. http://dx.doi. org/10.1016/0010-7824(92)90053-V

41. Brahmi D, Steenland MW, Renner RM, Gaffield ME, Curtis KM. Pregnancy outcomes with an IUD in situ: a systematic review. Contraception 2012;85:131-9. http://dx.doi.org/10.1016/j. contraception.2011.06.010

42. Tepper NK, Marchbanks PA, Curtis KM. Use of a checklist to rule out pregnancy: a systematic review. Contraception 2013;87:661-5. http:// dx.doi.org/10.1016/j.contraception.2012.08.007

43. Whiteman MK, Tyler CP, Folger SG, Gaffield ME, Curtis KM. When can a woman have an intrauterine device inserted? A systematic review. Contraception 2013;87:666-73. http://dx.doi.org/10.1016/j. contraception.2012.08.015

44. Teva Women's Health Inc. ParaGard T 380a intrauterine copper contraceptive [Prescribing information]. Sellersville, PA; 2013. http:// paragard.com/Pdf/ParaGard-PI.pdf

45. Bayer HealthCare Pharmaceuticals Inc. Mirena (levonorgestrel-releasing intrauterine system) [Prescribing information]. Whippany, NJ; 2014. http://labeling.bayerhealthcare.com/html/products/pi/Mirena_PI. pdf

46. Jatlaoui TC, Simmons KB, Curtis KM. The safety of intrauterine contraception initiation among women with current asymptomatic cervical infections or at increased risk of sexually transmitted infections. Contraception 2016. Epub June 1, 2016. http://dx.doi.org/10.1016/j. contraception.2016.05.013

47. Avonts D, Sercu M, Heyerick P, Vandermeeren I, Meheus A, Piot P. Incidence of uncomplicated genital infections in women using oral contraception or an intrauterine device: a prospective study. Sex Transm Dis 1990;17:23-9. http://dx.doi.org/10.1097/00007435-199017010-00006

48. Birgisson NE, Zhao Q, Secura GM, Madden T, Peipert JF. Positive testing for Neisseria gonorrhoeae and Chlamydia trachomatis and the risk of pelvic inflammatory disease in IUD users. J Womens Health (Larchmt) 2015;24:354-9. http://dx.doi.org/10.1089/jwh.2015.5190

49. Campbell SJ, Cropsey KL, Matthews CA. Intrauterine device use in a high-risk population: experience from an urban university clinic. Am J Obstet Gynecol 2007;197: 193.e1-6; discussion 193.e6-7.

50. Cropsey KL, Matthews C, Campbel S, Ivey S, Adawadkar S. Long-term, reversible contraception use among high-risk women treated in a university-based gynecology clinic: comparison between IUD and DepoProvera. J Womens Health (Larchmt) 2010;19:349-53. http://dx.doi. org/10.1089/jwh.2009.1518

51. Farley TMM, Rosenberg MJ, Rowe PJ, Chen JH, Meirik O. Intrauterine devices and pelvic inflammatory disease: an international perspective. Lancet 1992;339:785-8. http://dx.doi.org/10.1016/0140-6736(92)91904-M

52. Grimes DA, Schulz KF. Antibiotic prophylaxis for intrauterine contraceptive device insertion. Cochrane Database Syst Rev 2001;(2):CD001327.
53. LeFevre ML; US Preventive Services Task Force. Screening for chlamydia and gonorrhea: U.S. Preventive Services Task Force recommendation statement. Ann Intern Med 2014;161:902-10. http://dx.doi. org/10.7326/M14-1981

54. Mohllajee AP, Curtis KM, Peterson HB. Does insertion and use of an intrauterine device increase the risk of pelvic inflammatory disease among women with sexually transmitted infection? A systematic review. Contraception 2006;73:145-53. http://dx.doi.org/10.1016/j. contraception.2005.08.007

55. Morrison CS, Turner AN, Jones LB. Highly effective contraception and acquisition of HIV and other sexually transmitted infections. Best Pract Res Clin Obstet Gynaecol 2009;23:263-84. http://dx.doi.org/10.1016/j. bpobgyn.2008.11.004

56. Sufrin CB, Postlethwaite D, Armstrong MA, Merchant M, Wendt JM, Steinauer JE. Neisseria gonorrhea and Chlamydia trachomatis screening at intrauterine device insertion and pelvic inflammatory disease. Obstet Gynecol 2012;120:1314-21.

57. Tepper NK, Steenland MW, Marchbanks PA, Curtis KM. Laboratory screening prior to initiating contraception: a systematic review. Contraception 2013;87:645-9. http://dx.doi.org/10.1016/j. contraception.2012.08.009

58. Tepper NK, Steenland MW, Marchbanks PA, Curtis KM. Hemoglobin measurement prior to initiating copper intrauterine devices: a systematic review. Contraception 2013;87:639-44. http://dx.doi.org/10.1016/j. contraception.2012.08.008

59. Rivera R, Almonte H, Arreola M, et al. The effects of three different regimens of oral contraceptives and three different intrauterine devices on the levels of hemoglobin, serum iron and iron binding capacity in anemic women. Contraception 1983;27:311-27. http://dx.doi. org/10.1016/0010-7824(83)90009-4

60. Ursin G, Ross RK, Sullivan-Halley J, Hanisch R, Henderson B, Bernstein L. Use of oral contraceptives and risk of breast cancer in young women. Breast Cancer Res Treat 1998;50:175-84. http://dx.doi. org/10.1023/A:1006037823178

61. Hassan EO, el-Husseini M, el-Nahal N. The effect of 1-year use of the CuT 380A and oral contraceptive pills on hemoglobin and ferritin levels. Contraception 1999;60:101-5. http://dx.doi.org/10.1016/ S0010-7824(99)00065-7

62. Calzolari E, Guglielmo R, Viola F, Migliore L. Hematological parameters and iron therapy in women with IUDs. Experimental study [Italian]. Minerva Ginecol 1981;33:355-62.

63. Andersson K, Odlind V, Rybo G. Levonorgestrel-releasing and copperreleasing (Nova T) IUDs during five years of use: a randomized comparative trial. Contraception 1994;49:56-72. http://dx.doi. org/10.1016/0010-7824(94)90109-0

64. Andrade AT, Pizarro E, Shaw STJ Jr, Souza JP, Belsey EM, Rowe PJ; World Health Organization. Consequences of uterine blood loss caused by various intrauterine contraceptive devices in South American women. Contraception 1988;38:1-18. http://dx.doi. org/10.1016/0010-7824(88)90091-1

65. Blum M, Ariel J, Zacharowitch D. Ferritin, a faithful reflection of iron deficiency in IUD wearers with mild vaginal spotting. Adv Contracept 1991;7:39-42. http://dx.doi.org/10.1007/BF01850717

66. El-sheikha Z, Hamza A, Mahmoud M. Menstrual blood loss of TCu-380 A and TCu-200 B IUDs. Popul Sci 1990;July:55-62.

67. Gallegost AJ, Aznar R, Merino G, Guizer E. Intrauterine devices and menstrual blood loss. A comparative study of eight devices during the first six months of use. Contraception 1978;17:153-61. http://dx.doi. org/10.1016/0010-7824(78)90071-9

68. Gao J, Zeng S, Sun BL, et al. Menstrual blood loss, haemoglobin and ferritin concentration of Beijing women wearing steel ring, $\mathrm{VCu} 200$, and TCu 220c IUDs. Contraception 1986;34:559-71. http://dx.doi. org/10.1016/S0010-7824(86)80012-9 
69. Goh TH, Hariharan M. Effect of laparoscopic sterilization and insertion of Multiload $\mathrm{Cu} 250$ and Progestasert IUDs on serum ferritin levels. Contraception 1983;28:329-36. http://dx.doi. org/10.1016/0010-7824(83)90034-3

70. Goh TH, Hariharan M, Tan CH. A longitudinal study of serum iron indices and haemoglobin concentration following copperIUD insertion. Contraception 1980;22:389-95. http://dx.doi. org/10.1016/0010-7824(80)90024-4

71. Guillebaud J, Bonnar J, Morehead J, Matthews A. Menstrual blood-loss with intrauterine devices. Lancet 1976;1:387-90. http://dx.doi. org/10.1016/S0140-6736(76)90216-6

72. Haugan T, Skjeldestad FE, Halvorsen LE, Kahn H. A randomized trial on the clinical performance of Nova T380 and Gyne T380 Slimline copper IUDs. Contraception 2007;75:171-6. http://dx.doi. org/10.1016/j.contraception.2006.09.005

73. Kivijärvi A, Timonen H, Rajamäki A, Grönroos M. Iron deficiency in women using modern copper intrauterine devices. Obstet Gynecol 1986;67:95-8.

74. Larsson B, Hamberger L, Rybo G. Influence of copper intrauterine contraceptive devices (Cu-7-IUD) on the menstrual blood-loss. Acta Obstet Gynecol Scand 1975;54:315-8. http://dx.doi. org/10.3109/00016347509156760

75. Larsson G, Milsom I, Jonasson K, Lindstedt G, Rybo G. The long-term effects of copper surface area on menstrual blood loss and iron status in women fitted with an IUD. Contraception 1993;48:471-80. http:// dx.doi.org/10.1016/0010-7824(93)90136-U

76. Malmqvist R, Petersohn L, Bengtsson LP. Menstrual bleeding with copper-covered intrauterine contraceptive devices. Contraception 1974;9:627-33. http://dx.doi.org/10.1016/0010-7824(74)90048-1

77. Milsom I, Rybo G, Lindstedt G. The influence of copper surface area on menstrual blood loss and iron status in women fitted with an IUD. Contraception 1990;41:271-81. http://dx.doi. org/10.1016/0010-7824(90)90068-7

78. Piedras J, Córdova MS, Pérez-Toral MC, Lince E, Garza-Flores J. Predictive value of serum ferritin in anemia development after insertion of T Cu 220 intrauterine device. Contraception 1983;27:289-97. http:// dx.doi.org/10.1016/0010-7824(83)90007-0

79. Sivin I, Alvarez F, Diaz J, et al. Intrauterine contraception with copper and with levonorgestrel: a randomized study of the TCu 380Ag and levonorgestrel $20 \mathrm{mcg} /$ day devices. Contraception 1984;30:443-56. http://dx.doi.org/10.1016/0010-7824(84)90036-2

80. Sivin I, Stern J, Diaz J, et al. Two years of intrauterine contraception with levonorgestrel and with copper: a randomized comparison of the $\mathrm{TCu} 380 \mathrm{Ag}$ and levonorgestrel $20 \mathrm{mcg} /$ day devices. Contraception 1987;35:245-55. http://dx.doi.org/10.1016/0010-7824(87)90026-6

81. Tchai BS, Kim SW, Han JH, Im MW. Menstrual blood loss, iron nutriture, and the effects of Alza-T IPCS 52, T-Cu 220C and Lippes Loop D in Korean women. Seoul J Med 1987;28:51-9.

82. Wright EA, Kapu MM, Isichei UP. Zinc depletion and menorrhagia in Nigerians using copper T-200 intrauterine device. Trace Elem Med 1989;6:147-9.

83. Milsom I, Andersson K, Jonasson K, Lindstedt G, Rybo G. The influence of the Gyne-T 380S IUD on menstrual blood loss and iron status. Contraception 1995;52:175-9. http://dx.doi. org/10.1016/0010-7824(95)00163-5

84. CDC. Safe motherhood at a glance: Hyattsville, MD: CDC; 2015. http://www.cdc.gov/chronicdisease/resources/publications/aag/ pdf/2015/safe-motherhood-aag-2015.pdf

85. Cox S, Dietz P, Bowman B, Posner S. Prevalence of chronic conditions among women of reproductive age. Third National Summit on Preconception Health and Health Care: Tampa, FL; 2011.

86. Berenson AB, Rahman M, Wilkinson G. Effect of injectable and oral contraceptives on serum lipids. Obstet Gynecol 2009;114:786-94. http://dx.doi.org/10.1097/AOG.0b013e3181b76bea
87. Dilbaz B, Ozdegirmenci O, Caliskan E, Dilbaz S, Haberal A. Effect of etonogestrel implant on serum lipids, liver function tests and hemoglobin levels. Contraception 2010;81:510-4. http://dx.doi. org/10.1016/j.contraception.2010.01.014

88. Nelson AL, Cwiak C. Combined oral contraceptives. In: Hatcher RA, Trussel J, Nelson A, Cates W Jr, Kowal D, Policar M, editors. Contraceptive technology. 20th ed. New York, NY: Ardent Media; 2011:249-342.

89. Dragoman M, Curtis KM, Gaffield ME. Combined hormonal contraceptive use among women with known dyslipidemias: a systematic review of critical safety outcomes. Contraception 2015;:S0010-7824(15)00509-0.

90. US Department of Health and Human Services. Summary health statistics for U.S. adults: National Health Interview Survey, 2012. Report No. 0083-1972. Hyattsville, MD: US Department of Health and Human Services; 2014.

91. CDC. Surveillance for viral hepatitis_-United States, 2013. Atlanta, GA: CDC; 2013. http://www.cdc.gov/hepatitis/statistics/2013surveillance/ index.htm

92. Kohler BA, Sherman RL, Howlader N, et al. Annual report to the nation on the status of cancer, 1975-2011, featuring incidence of breast cancer subtypes by race/ethnicity, poverty, and state. J Natl Cancer Inst 2015;107:djv048. http://dx.doi.org/10.1093/jnci/djv048

93. Kapp N, Tilley IB, Curtis KM. The effects of hormonal contraceptive use among women with viral hepatitis or cirrhosis of the liver: a systematic review. Contraception 2009;80:381-6. http://dx.doi. org/10.1016/j.contraception.2009.04.007

94. Kapp N, Curtis KM. Hormonal contraceptive use among women with liver tumors: a systematic review. Contraception 2009;80:387-90. http://dx.doi.org/10.1016/j.contraception.2009.01.021

95. Tepper NK, Curtis KM, Steenland MW, Marchbanks PA. Physical examination prior to initiating hormonal contraception: a systematic review. Contraception 2013;87:650-4. http://dx.doi.org/10.1016/j. contraception.2012.08.010

96. National Cancer Institute. SEER Fast Stats. Washington DC: Surveillance Research Program, National Cancer Institute; 2015. http:// seer.cancer.gov/faststats

97. Watson M, Saraiya M, Benard V, et al. Burden of cervical cancer in the United States, 1998-2003. Cancer 2008;113(Suppl):2855-64. http:// dx.doi.org/10.1002/cncr.23756

98. National Center for Health Statistics, CDC. Health, United States, 2014: with special feature on adults. Hyattsville, MD: CDC; 2015.

99. European Study Group on Heterosexual Transmission of HIV. Comparison of female to male and male to female transmission of HIV in 563 stable couples. BMJ 1992;304:809-13. http://dx.doi. org/10.1136/bmj.304.6830.809

100. Coleman JS, Mwachari C, Balkus J, et al. Effect of the levonorgestrel intrauterine device on genital HIV-1 RNA shedding among HIV-1infected women not taking antiretroviral therapy in Nairobi, Kenya. J Acquir Immune Defic Syndr 2013;63:245-8. http://dx.doi. org/10.1097/QAI.0b013e31828decf8

101. Haddad LB, Cwiak C, Jamieson DJ, et al. Contraceptive adherence among HIV-infected women in Malawi: a randomized controlled trial of the copper intrauterine device and depot medroxyprogesterone acetate. Contraception 2013;88:737-43. http://dx.doi.org/10.1016/j. contraception.2013.08.006 
102. Heikinheimo O, Lehtovirta P, Aho I, Ristola M, Paavonen J. The levonorgestrel-releasing intrauterine system in human immunodeficiency virus-infected women: a 5-year follow-up study. Am J Obstet Gynecol 2011;204:126 e1-4.

103. Heikinheimo O, Lehtovirta P, Suni J, Paavonen J. The levonorgestrelreleasing intrauterine system (LNG-IUS) in HIV-infected womeneffects on bleeding patterns, ovarian function and genital shedding of HIV. Hum Reprod 2006;21:2857-61. http://dx.doi.org/10.1093/ humrep/del264

104. Kakaire O, Byamugisha JK, Tumwesigye NM, Gemzell-Danielsson K. Clinical versus laboratory screening for sexually transmitted infections prior to insertion of intrauterine contraception among women living with HIV/AIDS: a randomized controlled trial. Hum Reprod 2015;30:1573-9. http://dx.doi.org/10.1093/humrep/dev109

105. Kovacs A, Wasserman SS, Burns D, et al; DATRI Study Group; WIHS Study Group. Determinants of HIV-1 shedding in the genital tract of women. Lancet 2001;358:1593-601. http://dx.doi.org/10.1016/ S0140-6736(01)06653-3

106. Landolt NK, Phanuphak N, Teeratakulpisarn N, et al. Uptake and continuous use of copper intrauterine device in a cohort of HIV-positive women. AIDS Care 2013;25:710-4. http://dx.doi.org/10.1080/0954 0121.2012 .752786

107. Lehtovirta P, Paavonen J, Heikinheimo O. Experience with the levonorgestrel-releasing intrauterine system among HIV-infected women. Contraception 2007;75:37-9. http://dx.doi.org/10.1016/j. contraception.2006.09.006

108. Morrison CS, Sekadde-Kigondu C, Sinei SK, Weiner DH, Kwok C, Kokonya D. Is the intrauterine device appropriate contraception for HIV-1-infected women? BJOG 2001;108:784-90. http://dx.doi. org/10.1111/j.1471-0528.2001.00204.x

109. Mostad SB, Overbaugh J, DeVange DM, et al. Hormonal contraception, vitamin A deficiency, and other risk factors for shedding of HIV-1 infected cells from the cervix and vagina. Lancet 1997;350:922-7. http://dx.doi.org/10.1016/S0140-6736(97)04240-2

110. Richardson BA, Morrison CS, Sekadde-KigonduC, etal.Effectofintrauterine device use on cervical shedding of HIV-1 DNA. AIDS 1999;13:2091-7. http://dx.doi.org/10.1097/00002030-199910220-00012

111. Sinei SK, Morrison CS, Sekadde-Kigondu C, Allen M, Kokonya D. Complications of use of intrauterine devices among HIV-1-infected women. Lancet 1998;351:1238-41. http://dx.doi.org/10.1016/ S0140-6736(97)10319-1

112. Stringer EM, Kaseba C, Levy J, Sinkala M, Goldenberg RL, Chi BH, et al. A randomized trial of the intrauterine contraceptive device vs hormonal contraception in women who are infected with the human immunodeficiency virus. Am J Obstet Gynecol 2007;197:144 e1-8.

113. Stringer EM, Levy J, Sinkala M, et al. HIV disease progression by hormonal contraceptive method: secondary analysis of a randomized trial. AIDS 2009;23:1377-82. http://dx.doi.org/10.1097/ QAD.0b013e32832cbca8

114. Tepper NK, Curtis KM, Nanda K, Jamieson DJ. Safety of intrauterine devices among women with HIV: a systematic review. Contraception 2016. Epub June 22, 2016. http://dx.doi.org/10.1016/j.contraception.2016.06.011

115. Lopez LM, Bernholc A, Zeng Y, et al. Interventions for pain with intrauterine device insertion. Cochrane Database Syst Rev 2015;7:CD007373.

116. Zapata LB, Jatlaoui TC, Marchbanks PA, Curtis KM. Medications to ease intrauterine device insertion: a systematic review. Contraception 2016. Epub June 29, 2016. http://dx.doi.org/10.1016/j.contraception.2016.06.014
117. Bahamondes MV, Espejo-Arce X, Bahamondes L. Effect of vaginal administration of misoprostol before intrauterine contraceptive insertion following previous insertion failure: a double blind RCT. Hum Reprod 2015;30:1861-6. http://dx.doi.org/10.1093/humrep/ $\operatorname{dev} 137$

118. Cirik D, Taskin E, Tuglu A, Ortac A, Dai O. Paracervical block with $1 \%$ lidocaine for pain control during intrauterine device insertion: a prospective, single-blinded, controlled study. Int J Reprod Contracept Obstet Gynecol 2013;2:263-7.

119. Mody SK, Kiley J, Rademaker A, Gawron L, Stika C, Hammond C. Pain control for intrauterine device insertion: a randomized trial of $1 \%$ lidocaine paracervical block. Contraception 2012;86:704-9. http:// dx.doi.org/10.1016/j.contraception.2012.06.004

120. Grimes DA, Schulz KF. Prophylactic antibiotics for intrauterine device insertion: a metaanalysis of the randomized controlled trials. Contraception 1999;60:57-63. http://dx.doi.org/10.1016/ S0010-7824(99)00071-2

121. Wilson W, Taubert KA, Gewitz M, et al; American Heart Association Rheumatic Fever, Endocarditis, and Kawasaki Disease Committee; American Heart Association Council on Cardiovascular Disease in the Young; American Heart Association Council on Clinical Cardiology; American Heart Association Council on Cardiovascular Surgery and Anesthesia; Quality of Care and Outcomes Research Interdisciplinary Working Group. Prevention of infective endocarditis: guidelines from the American Heart Association: a guideline from the American Heart Association Rheumatic Fever, Endocarditis, and Kawasaki Disease Committee, Council on Cardiovascular Disease in the Young, and the Council on Clinical Cardiology, Council on Cardiovascular Surgery and Anesthesia, and the Quality of Care and Outcomes Research Interdisciplinary Working Group. Circulation 2007;116:1736-54. http://dx.doi.org/10.1161/CIRCULATIONAHA.106.183095

122. Steenland MW, Zapata LB, Brahmi D, Marchbanks PA, Curtis KM. The effect of follow-up visits or contacts after contraceptive initiation on method continuation and correct use. Contraception 2013;87:62530. http://dx.doi.org/10.1016/j.contraception.2012.09.018

123. Steenland MW, Zapata LB, Brahmi D, Marchbanks PA, Curtis KM. Appropriate follow up to detect potential adverse events after initiation of select contraceptive methods: a systematic review. Contraception 2013;87:611-24. http://dx.doi.org/10.1016/j. contraception.2012.09.017

124. Canto De Cetina TE, Canto P, Ordoñez Luna M. Effect of counseling to improve compliance in Mexican women receiving depotmedroxyprogesterone acetate. Contraception 2001;63:143-6. http:// dx.doi.org/10.1016/S0010-7824(01)00181-0

125. Lei ZW, Wu SC, Garceau RJ, et al. Effect of pretreatment counseling on discontinuation rates in Chinese women given depomedroxyprogesterone acetate for contraception. Contraception 1996;53:357-61. http://dx.doi.org/10.1016/0010-7824(96)00085-6

126. Godfrey EM, Folger SG, Jeng G, Jamieson DJ, Curtis KM. Treatment of bleeding irregularities in women with copper-containing IUDs: a systematic review. Contraception 2013;87:549-66. http://dx.doi. org/10.1016/j.contraception.2012.09.006

127. Toppozada M, Anwar M, Abdel Rahman H, Gaweesh S. Control of IUD-induced bleeding by three non-steroidal anti-inflammatory drugs. Contracept Deliv Syst 1982;3:117-25.

128. Toppozada M, El-Attar A, El-Ayyat MA, Khamis Y. Management of uterine bleeding by PGs or their synthesis inhibitors. Adv Prostaglandin Thromboxane Res 1980;8:1459-63. 
129. Wu S, Wang C, Cheng W, et al. Randomized multi-center study of baofuxin for treatment of bleeding side-effect induced by IUD. Reprod Contracept 2000;11:152-7.

130. Mercorio F, De Simone R, Di Carlo C, et al. Effectiveness and mechanism of action of desmopressin in the treatment of copper intrauterine device-related menorrhagia: a pilot study. Hum Reprod 2003;18:2319-22. http://dx.doi.org/10.1093/humrep/deg449

131. Pedron N, Lozano M, Aznar R. Treatment of hypermenorrhea with mefenamic acid in women using IUDs. Contracept Deliv Syst 1982;3:135-9.

132. Pizarro E, Mehech G, Hidalgo M, Muñoz G, Romero C. Effect of meclofenamic acid on menstruation in hypermenorrheic women using intrauterine devices [Spanish]. Rev Chil Obstet Ginecol 1988;53:43-56.

133. Chinese National IUD Research Working Group. Prevention and treatment of IUD-induced menorrhagia with antifibrinolytic and antiprostaglandin drugs [Chinese]. Zhonghua Fu Chan Ke Za Zhi 1987;22:291-4, 312.

134. Di Lieto A, Catalano D, Miranda L, Paladini A. Action of a prostaglandin synthetase inhibitor on IUD associated uterine bleeding. Clin Exp Obstet Gynecol 1987;14:41-4.

135. Ylikorkala O, Viinikka L. Comparison between antifibrinolytic and antiprostaglandin treatment in the reduction of increased menstrual blood loss in women with intrauterine contraceptive devices. Br J Obstet Gynaecol 1983;90:78-83. http://dx.doi. org/10.1111/j.1471-0528.1983.tb06751.x

136. US Food and Drug Administration. Cyklokapron: tranexamic acid injection [Prescribing information]. New York, NY; 2011.

137. US Food and Drug Administration. Lysteda: tranexamic acid tablets [Prescribing information]. Parsippany; NJ. 2011.

138. Pedrón N, Lozano M, Gallegos AJ. The effect of acetylsalicylic acid on menstrual blood loss in women with IUDs. Contraception 1987;36:295-303. http://dx.doi.org/10.1016/0010-7824(87)90099-0

139. Hidalgo M, Bahamondes L, Perrotti M, Diaz J, Dantas-Monteiro C, Petta C. Bleeding patterns and clinical performance of the levonorgestrel-releasing intrauterine system (Mirena) up to two years. Contraception 2002;65:129-32. http://dx.doi.org/10.1016/ S0010-7824(01)00302-X

140. Tepper NK, Steenland MW, Gaffield ME, Marchbanks PA, Curtis KM. Retention of intrauterine devices in women who acquire pelvic inflammatory disease: a systematic review. Contraception 2013;87:65560. http://dx.doi.org/10.1016/j.contraception.2012.08.011

141. Larsson B, Wennergren M. Investigation of a copper-intrauterine device (Cu-IUD) for possible effect on frequency and healing of pelvic inflammatory disease. Contraception 1977;15:143-9. http://dx.doi. org/10.1016/0010-7824(77)90012-9

142. Söderberg G, Lindgren S. Influence of an intrauterine device on the course of an acute salpingitis. Contraception 1981;24:137-43. http:// dx.doi.org/10.1016/0010-7824(81)90086-X

143. Teisala K. Removal of an intrauterine device and the treatment of acute pelvic inflammatory disease. Ann Med 1989;21:63-5. http://dx.doi. org/10.3109/07853898909149184

144. Altunyurt S, Demir N, Posaci C. A randomized controlled trial of coil removal prior to treatment of pelvic inflammatory disease. Eur J Obstet Gynecol Reprod Biol 2003;107:81-4. http://dx.doi.org/10.1016/ S0301-2115(02)00342-1
145. Stewart FH, Harper CC, Ellertson CE, Grimes DA, Sawaya GF, Trussell J. Clinical breast and pelvic examination requirements for hormonal contraception: current practice vs evidence. JAMA 2001;285:2232-9. http://dx.doi.org/10.1001/jama.285.17.2232

146. Mansour D, Korver T, Marintcheva-Petrova M, Fraser IS. The effects of Implanon on menstrual bleeding patterns. Eur J Contracept Reprod Health Care 2008;13(Suppl 1):13-28. http://dx.doi. org/10.1080/13625180801959931

147. Abdel-Aleem H, d'Arcangues C, Vogelsong K, Gülmezoglu AM. Treatment of vaginal bleeding irregularities induced by progestin only contraceptives. Cochrane Database Syst Rev 2007;(2):CD003449.

148. Archer DF, Philput CB, Levine AS, et al. Effects of ethinyl estradiol and ibuprofen compared to placebo on endometrial bleeding, cervical mucus and the postcoital test in levonorgestrel subcutaneous implant users. Contraception 2008;78:106-12. http://dx.doi.org/10.1016/j. contraception.2008.04.003

149. Buasang K, Taneepanichskul S. Efficacy of celecoxib on controlling irregular uterine bleeding secondary to Jadelle use. J Med Assoc Thai 2009;92:301-7.

150. Phaliwong P, Taneepanichskul S. The effect of mefenamic acid on controlling irregular uterine bleeding second to Implanon use. J Med Assoc Thai 2004;87(Suppl 3):S64-8.

151. Weisberg E, Hickey M, Palmer D, et al. A randomized controlled trial of treatment options for troublesome uterine bleeding in Implanon users. Hum Reprod 2009;24:1852-61. http://dx.doi.org/10.1093/ humrep/dep081

152. Weisberg E, Hickey M, Palmer D, et al. A pilot study to assess the effect of three short-term treatments on frequent and/or prolonged bleeding compared to placebo in women using Implanon. Hum Reprod 2006;21:295-302. http://dx.doi.org/10.1093/humrep/dei273

153. Cheng L, Zhu H, Wang A, Ren F, Chen J, Glasier A. Once a month administration of mifepristone improves bleeding patterns in women using subdermal contraceptive implants releasing levonorgestrel. Hum Reprod 2000;15:1969-72. http://dx.doi.org/10.1093/humrep/15.9.1969

154. Alvarez-Sanchez F, Brache V, Thevenin F, Cochon L, Faundes A. Hormonal treatment for bleeding irregularities in Norplant implant users. Am J Obstet Gynecol 1996;174:919-22. http://dx.doi. org/10.1016/S0002-9378(96)70326-5

155. Díaz S, Croxatto HB, Pavez M, Belhadj H, Stern J, Sivin I. Clinical assessment of treatments for prolonged bleeding in users of Norplant implants. Contraception 1990;42:97-109. http://dx.doi. org/10.1016/0010-7824(90)90094-C

156. Witjaksono J, Lau TM, Affandi B, Rogers PA. Oestrogen treatment for increased bleeding in Norplant users: preliminary results. Hum Reprod 1996;11(Suppl 2):109-14. http://dx.doi.org/10.1093/ humrep/11.suppl_2.109

157. Abdel-Aleem H, Shaaban OM, Amin AF, Abdel-Aleem AM. Tamoxifen treatment of bleeding irregularities associated with Norplant use. Contraception 2005;72:432-7. http://dx.doi.org/10.1016/j. contraception.2005.05.015

158. Phupong V, Sophonsritsuk A, Taneepanichskul S. The effect of tranexamic acid for treatment of irregular uterine bleeding secondary to Norplant use. Contraception 2006;73:253-6. http://dx.doi. org/10.1016/j.contraception.2005.09.012

159. d'Arcangues C, Piaggio G, Brache V, et al; Study Group on Progestogeninduced Vaginal Bleeding Disturbances. Effectiveness and acceptability of vitamin $\mathrm{E}$ and low-dose aspirin, alone or in combination, on Norplant-induced prolonged bleeding. Contraception 2004;70:45162. http://dx.doi.org/10.1016/j.contraception.2004.05.012 
160. Subakir SB, Setiadi E, Affandi B, Pringgoutomo S, Freisleben HJ. Benefits of vitamin E supplementation to Norplant users - in vitro and in vivo studies. Toxicology 2000;148:173-8. http://dx.doi.org/10.1016/ S0300-483X(00)00208-0

161. Kapp N, Gaffield ME. Initiation of progestogen-only injectables on different days of the menstrual cycle and its effect on contraceptive effectiveness and compliance: a systematic review. Contraception 2013;87:576-82. http://dx.doi.org/10.1016/j.contraception.2012.08.017

162. Petta CA, Faúndes A, Dunson TR, et al. Timing of onset of contraceptive effectiveness in Depo-Provera users. II. Effects on ovarian function. Fertil Steril 1998;70:817-20. http://dx.doi.org/10.1016/ S0015-0282(98)00309-4

163. Petta CA, Faundes A, Dunson TR, et al. Timing of onset of contraceptive effectiveness in Depo-Provera users: Part I. Changes in cervical mucus. Fertil Steril 1998;69:252-7. http://dx.doi.org/10.1016/ S0015-0282(97)00477-9

164. Siriwongse T, Snidvongs W, Tantayaporn P, Leepipatpaiboon S. Effect of depo-medroxyprogesterone acetate on serum progesterone levels when administered on various cycle days. Contraception 1982;26:48793. http://dx.doi.org/10.1016/0010-7824(82)90147-0

165. Balkus J, Miller L. Same-day administration of depot-medroxyprogesterone acetate injection: a retrospective chart review. Contraception 2005;71:395-8. http://dx.doi.org/10.1016/j.contraception.2004.10.014

166. Morroni C, Grams M, Tiezzi L, Westhoff C. Immediate monthly combination contraception to facilitate initiation of the depot medroxyprogesterone acetate contraceptive injection. Contraception 2004;70:19-23. http://dx.doi.org/10.1016/j.contraception.2004.02.007

167. Nelson AL, Katz T. Initiation and continuation rates seen in 2-year experience with Same Day injections of DMPA. Contraception 2007;75:84-7. http://dx.doi.org/10.1016/j.contraception.2006.09.007

168. Rickert VI, Tiezzi L, Lipshutz J, León J, Vaughan RD, Westhoff C. Depo Now: preventing unintended pregnancies among adolescents and young adults. J Adolesc Health 2007;40:22-8. http://dx.doi. org/10.1016/j.jadohealth.2006.10.018

169. Sneed R, Westhoff C, Morroni C, Tiezzi L. A prospective study of immediate initiation of depo medroxyprogesterone acetate contraceptive injection. Contraception 2005;71:99-103. http://dx.doi.org/10.1016/j. contraception.2004.08.014

170. Tepper NK, Curtis KM, Steenland MW, Marchbanks PA. Blood pressure measurement prior to initiating hormonal contraception: a systematic review. Contraception 2013;87:631-8. http://dx.doi. org/10.1016/j.contraception.2012.08.025

171. Diab KM, Zaki MM. Contraception in diabetic women: comparative metabolic study of Norplant, depot medroxyprogesterone acetate, low dose oral contraceptive pill and CuT380A. J Obstet Gynaecol Res 2000;26:1726. http://dx.doi.org/10.1111/j.1447-0756.2000.tb01195.x

172. Garg SK, Chase HP, Marshall G, Hoops SL, Holmes DL, Jackson WE. Oral contraceptives and renal and retinal complications in young women with insulin-dependent diabetes mellitus. JAMA 1994;271:1099-102. http://dx.doi.org/10.1001/jama.1994.03510380055037

173. Grigoryan OR, Grodnitskaya EE, Andreeva EN, Chebotnikova TV, Melnichenko GA. Use of the NuvaRing hormone-releasing system in late reproductive-age women with type 1 diabetes mellitus. Gynecol Endocrinol 2008;24:99-104. http://dx.doi.org/10.1080/09513590701708795

174. Kahn HS, Curtis KM, Marchbanks PA. Effects of injectable or implantable progestin-only contraceptives on insulin-glucose metabolism and diabetes risk. Diabetes Care 2003;26:216-25. http:// dx.doi.org/10.2337/diacare.26.1.216
175. Lopez LM, Grimes DA, Schulz KF. Steroidal contraceptives: effect on carbohydrate metabolism in women without diabetes mellitus. Cochrane Database Syst Rev 2009; (4):CD006133.

176. Rogovskaya S, Rivera R, Grimes DA, et al. Effect of a levonorgestrel intrauterine system on women with type 1 diabetes: a randomized trial. Obstet Gynecol 2005;105:811-5. http://dx.doi.org/10.1097/01. AOG.0000156301.11939.56

177. Troisi RJ, Cowie CC, Harris MI. Oral contraceptive use and glucose metabolism in a national sample of women in the United States. Am J Obstet Gynecol 2000;183:389-95. http://dx.doi.org/10.1067/mob.2000.105909

178. Bonny AE, Secic M, Cromer B. Early weight gain related to later weight gain in adolescents on depot medroxyprogesterone acetate. Obstet Gynecol 2011;117:793-7. http://dx.doi.org/10.1097/AOG.0b013e31820f387c

179. LeYC, Rahman M, Berenson AB. Early weight gain predicting later weight gain among depot medroxyprogesterone acetate users. Obstet Gynecol 2009;114:279-84. http://dx.doi.org/10.1097/AOG.0b013e3181af68b2

180. Risser WL, Gefter LR, Barratt MS, Risser JM. Weight change in adolescents who used hormonal contraception. J Adolesc Health 1999;24:433-6. http://dx.doi.org/10.1016/S1054-139X(98)00151-7

181. Paulen ME, Curtis KM. When can a woman have repeat progestogenonly injectables - depot medroxyprogesterone acetate or norethisterone enantate? Contraception 2009;80:391-408. http://dx.doi. org/10.1016/j.contraception.2009.03.023

182. Pardthaisong T. Return of fertility after use of the injectable contraceptive Depo Provera: up-dated data analysis. J Biosoc Sci 1984;16:23-34. http://dx.doi.org/10.1017/S0021932000014760

183. Schwallie PC, Assenzo JR. The effect of depo-medroxyprogesterone acetate on pituitary and ovarian function, and the return of fertility following its discontinuation: a review. Contraception 1974;10:181202. http://dx.doi.org/10.1016/0010-7824(74)90073-0

184. Steiner MJ, Kwok C, Stanback J, et al. Injectable contraception: what should the longest interval be for reinjections? Contraception 2008;77:410-4. http://dx.doi.org/10.1016/j.contraception.2008.01.017

185. Bassol S, Garza-Flores J, Cravioto MC, et al. Ovarian function following a single administration of depo-medroxyprogesterone acetate (DMPA) at different doses. Fertil Steril 1984;42:216-22.

186. Fotherby K, Koetsawang S, Mathrubutham M. Pharmacokinetic study of different doses of Depo Provera. Contraception 1980;22:527-36. http://dx.doi.org/10.1016/0010-7824(80)90105-5

187. Fotherby K, Saxena BN, Shrimanker K, et al. A preliminary pharmacokinetic and pharmacodynamic evaluation of depotmedroxyprogesterone acetate and norethisterone oenanthate. Fertil Steril 1980;34:131-9.

188. Garza-Flores J, Cardenas S, Rodríguez V, Cravioto MC, Diaz-Sanchez V, Perez-Palacios G. Return to ovulation following the use of long-acting injectable contraceptives: a comparative study. Contraception 1985;31:361-6. http://dx.doi.org/10.1016/0010-7824(85)90004-6

189. Jain J, Dutton C, Nicosia A, Wajszczuk C, Bode FR, Mishell DR Jr. Pharmacokinetics, ovulation suppression and return to ovulation following a lower dose subcutaneous formulation of Depo-Provera. Contraception 2004;70:11-8. http://dx.doi.org/10.1016/j. contraception.2004.01.011

190. Lan PT, Aedo AR, Landgren BM, Johannisson E, Diczfalusy E. Return of ovulation following a single injection of depo-medroxyprogesterone acetate: a pharmacokinetic and pharmacodynamic study. Contraception 1984;29:1-18. http://dx.doi.org/10.1016/0010-7824(84)90054-4 
191. Ortiz A, Hirol M, Stanczyk FZ, Goebelsmann U, Mishell DR Jr. Serum medroxyprogesterone acetate (MPA) concentrations and ovarian function following intramuscular injection of depo-MPA. J Clin Endocrinol Metab 1977;44:32-8. http://dx.doi.org/10.1210/jcem-44-1-32

192. Saxena BN, Dusitsin N, Tankeyoon M, Chaudhury RR. Return of ovulation after the cessation of depot-medroxy progesterone acetate treatment in Thai women. J Med Assoc Thai 1980;63:66-9.

193. Toh YC, Jain J, Rahnny MH, Bode FR, Ross D. Suppression of ovulation by a new subcutaneous depot medroxyprogesterone acetate $(104 \mathrm{mg} / 0.65 \mathrm{~mL})$ contraceptive formulation in Asian women. Clin Ther 2004;26:1845-54. http://dx.doi.org/10.1016/j. clinthera.2004.11.013

194. Arias RD, Jain JK, Brucker C, Ross D, Ray A. Changes in bleeding patterns with depot medroxyprogesterone acetate subcutaneous injection $104 \mathrm{mg}$. Contraception 2006;74:234-8. http://dx.doi. org/10.1016/j.contraception.2006.03.008

195. Dempsey A, Roca C, WesthoffC. Vaginal estrogen supplementation during Depo-Provera initiation: a randomized controlled trial. Contraception 2010;82:250-5. http://dx.doi.org/10.1016/j.contraception.2010.04.003

196. Abdel-Aleem H, d'Arcangues C, Vogelsong KM, Gülmezoglu AM. Treatment of vaginal bleeding irregularities induced by progestin only contraceptives. Cochrane Database Syst Rev 2007; (4):CD003449.

197. Harel Z, Biro F, Kollar L, Riggs S, Flanagan P, Vaz R. Supplementation with vitamin $\mathrm{C}$ and/or vitamin $\mathrm{B}(6)$ in the prevention of Depo-Provera side effects in adolescents. J Pediatr Adolesc Gynecol 2002;15:153-8. http://dx.doi.org/10.1016/S1083-3188(02)00148-1

198. Nathirojanakun P, Taneepanichskul S, Sappakitkumjorn N. Efficacy of a selective COX-2 inhibitor for controlling irregular uterine bleeding in DMPA users. Contraception 2006;73:584-7. http://dx.doi. org/10.1016/j.contraception.2005.09.013

199. Tantiwattanakul P, Taneepanichskul S. Effect of mefenamic acid on controlling irregular uterine bleeding in DMPA users. Contraception 2004;70:277-9. http://dx.doi.org/10.1016/j.contraception.2004.04.003

200. Said S, Sadek W, Rocca M, et al. Clinical evaluation of the therapeutic effectiveness of ethinyl oestradiol and oestrone sulphate on prolonged bleeding in women using depot medroxyprogesterone acetate for contraception. World Health Organization, Special Programme of Research, Development and Research Training in Human Reproduction, Task Force on Long-acting Systemic Agents for Fertility Regulation. Hum Reprod 1996;11(Suppl 2):1-13. http://dx.doi.org/10.1093/humrep/11.suppl_2.1

201. Sadeghi-Bazargani H, Ehdaeivand F, Arshi S, Eftekhar H, Sezavar H, Amanati L. Low-dose oral contraceptive to re-induce menstrual bleeding in amenorrheic women on DMPA treatment: a randomized clinical trial. Med Sci Monit 2006;12:CR420-5.

202. Brahmi D, Curtis KM. When can a woman start combined hormonal contraceptives (CHCs)? A systematic review. Contraception 2013;87:524 38. http://dx.doi.org/10.1016/j.contraception.2012.09.010

203. Edwards SM, Zieman M, Jones K, Diaz A, Robilotto C, Westhoff C. Initiation of oral contraceptives-start now! J Adolesc Health 2008;43:432-6. http://dx.doi.org/10.1016/j.jadohealth.2008.06.008

204. Westhoff C, Heartwell S, Edwards S, et al. Initiation of oral contraceptives using a quick start compared with a conventional start: a randomized controlled trial. Obstet Gynecol 2007;109:1270-6. http://dx.doi.org/10.1097/01.AOG.0000264550.41242.f2

205. Westhoff C, Morroni C, Kerns J, Murphy PA. Bleeding patterns after immediate vs. conventional oral contraceptive initiation: a randomized, controlled trial. Fertil Steril 2003;79:322-9. http://dx.doi.org/10.1016/ S0015-0282(02)04680-0
206. Baerwald AR, Olatunbosun OA, Pierson RA. Ovarian follicular development is initiated during the hormone-free interval of oral contraceptive use. Contraception 2004;70:371-7. http://dx.doi. org/10.1016/j.contraception.2004.05.006

207. Baerwald AR, Pierson RA. Ovarian follicular development during the use of oral contraception: a review. J Obstet Gynaecol Can 2004;26:1924. http://dx.doi.org/10.1016/S1701-2163(16)30692-2

208. Duijkers IJM, Klipping C, Verhoeven CHJ, Dieben TOM. Ovarian function with the contraceptive vaginal ring or an oral contraceptive: a randomized study. Hum Reprod 2004;19:2668-73. http://dx.doi. org/10.1093/humrep/deh493

209. Killick S, Eyong E, Elstein M. Ovarian follicular development in oral contraceptive cycles. Fertil Steril 1987;48:409-13.

210. Molloy BG, Coulson KA, Lee JM, Watters JK. "Missed pill" conception: fact or fiction? Br Med J (Clin Res Ed) 1985;290:1474-5. http://dx.doi. org/10.1136/bmj.290.6480.1474-a

211. Mulders TM, Dieben TO, Bennink HJ. Ovarian function with a novel combined contraceptive vaginal ring. Hum Reprod 2002;17:2594-9. http://dx.doi.org/10.1093/humrep/17.10.2594

212. Schwartz JL, Creinin MD, Pymar HC, Reid L. Predicting risk of ovulation in new start oral contraceptive users. Obstet Gynecol 2002;99:177-82.

213. Sitavarin S, Jaisamrarn U, Taneepanichskul S. A randomized trial on the impact of starting day on ovarian follicular activity in very low dose oral contraceptive pills users. J Med Assoc Thai 2003;86:442-8.

214. Taylor DR, Anthony FW, Dennis KJ. Suppression of ovarian function by Microgynon 30 in day 1 and day 5 "starters." Contraception 1986;33:463-71. http://dx.doi.org/10.1016/S0010-7824(86)80005-1

215. Smith SK, Kirkman RJ, Arce BB, McNeilly AS, Loudon NB, Baird DT. The effect of deliberate omission of Trinordiol or Microgynon on the hypothalamo-pituitary-ovarian axis. Contraception 1986;34:51322. http://dx.doi.org/10.1016/0010-7824(86)90060-0

216. Lara-Torre E, Schroeder B. Adolescent compliance and side effects with Quick Start initiation of oral contraceptive pills. Contraception 2002;66:81-5. http://dx.doi.org/10.1016/S0010-7824(02)00326-8

217. Murthy AS, Creinin MD, Harwood B, Schreiber CA. Same-day initiation of the transdermal hormonal delivery system (contraceptive patch) versus traditional initiation methods. Contraception 2005;72:333-6. http://dx.doi.org/10.1016/j.contraception.2005.05.009

218. Westhoff C, Osborne LM, Schafer JE, Morroni C. Bleeding patterns after immediate initiation of an oral compared with a vaginal hormonal contraceptive. Obstet Gynecol 2005;106:89-96. http://dx.doi. org/10.1097/01.AOG.0000164483.13326.59

219. Yeshaya A, Orvieto R, Kaplan B, et al. Flexible starting schedule for oral contraception: effect on the incidence of breakthrough bleeding and compliance. Eur J Contracept Reprod Health Care 1998;3:121-3. http://dx.doi.org/10.3109/13625189809051414

220. Yeshaya A, Orvieto R, Kauschansky A, et al. A delayed starting schedule of oral contraception: the effect on the incidence of breakthrough bleeding and compliance in women. Eur J Contracept Reprod Health Care 1996;1:263-5. http://dx.doi.org/10.3109/13625189609150668

221. Westhoff C, Kerns J, Morroni C, Cushman LF, Tiezzi L, Murphy PA. Quick start: novel oral contraceptive initiation method. Contraception 2002;66:141-5. http://dx.doi.org/10.1016/S0010-7824(02)00351-7

222. WHO Collaborative Study of Cardiovascular Disease and Steroid Hormone Contraception. Acute myocardial infarction and combined oral contraceptives: results of an international multicentre case-control study. Lancet 1997;349:1202-9. http://dx.doi.org/10.1016/ S0140-6736(97)02358-1 
223. Dunn N, Thorogood M, Faragher B, et al. Oral contraceptives and myocardial infarction: results of the MICA case-control study. BMJ 1999;318:1579-83. http://dx.doi.org/10.1136/bmj.318.7198.1579

224. Lewis MA, Heinemann LA, Spitzer WO, MacRae KD, Bruppacher R. The use of oral contraceptives and the occurrence of acute myocardial infarction in young women. Results from the Transnational Study on Oral Contraceptives and the Health of Young Women. Contraception 1997;56:129-40. http://dx.doi.org/10.1016/S0010-7824(97)00118-2

225. WHO Collaborative Study of Cardiovascular Disease and Steroid Hormone Contraception. Ischaemic stroke and combined oral contraceptives: results of an international, multicentre, case-control study. Lancet 1996;348:498-505. http://dx.doi.org/10.1016/ S0140-6736(95) 12393-8

226. Heinemann LA, Lewis MA, Spitzer WO, Thorogood M, GuggenmoosHolzmann I, Bruppacher R; Transnational Research Group on Oral Contraceptives and the Health of Young Women. Thromboembolic stroke in young women. A European case-control study on oral contraceptives. Contraception 1998;57:29-37. http://dx.doi. org/10.1016/S0010-7824(97)00204-7

227. WHO Collaborative Study of Cardiovascular Disease and Steroid Hormone Contraception. Haemorrhagic stroke, overall stroke risk, and combined oral contraceptives: results of an international, multicentre, case-control study. Lancet 1996;348:505-10. http:// dx.doi.org/10.1016/S0140-6736(95)12394-6

228. Mohllajee AP, Curtis KM, Martins SL, Peterson HB. Does use of hormonal contraceptives among women with thrombogenic mutations increase their risk of venous thromboembolism? A systematic review. Contraception 2006;73:166-78. http://dx.doi.org/10.1016/j. contraception.2005.08.011

229. Wu O, Robertson L, Twaddle S, et al. Screening for thrombophilia in high-risk situations: systematic review and cost-effectiveness analysis. The Thrombosis: Risk and Economic Assessment of Thrombophilia Screening (TREATS) study. Health Technol Assess 2006;10:1-110. http://dx.doi.org/10.3310/hta 10110

230. Wu O, Greer IA. Is screening for thrombophilia cost-effective? Curr Opin Hematol 2007;14:500-3. http://dx.doi.org/10.1097/ MOH.0b013e32825f5318

231. Blickstein D, Blickstein I. Oral contraception and thrombophilia. Curr Opin Obstet Gynecol 2007;19:370-6. http://dx.doi.org/10.1097/ GCO.0b013e32821642e6

232. Steenland MW, Rodriguez MI, Marchbanks PA, Curtis KM. How does the number of oral contraceptive pill packs dispensed or prescribed affect continuation and other measures of consistent and correct use? A systematic review. Contraception 2013;87:605-10. http://dx.doi. org/10.1016/j.contraception.2012.08.004

233. White KO, Westhoff C. The effect of pack supply on oral contraceptive pill continuation: a randomized controlled trial. Obstet Gynecol 2011;118:615-22. http://dx.doi.org/10.1097/ AOG.0b013e3182289eab

234. Foster DG, Parvataneni R, de Bocanegra HT, Lewis C, Bradsberry M, Darney P. Number of oral contraceptive pill packages dispensed, method continuation, and costs. Obstet Gynecol 2006;108:1107-14. http://dx.doi.org/10.1097/01.AOG.0000239122.98508.39

235. Foster DG, Hulett D, Bradsberry M, Darney P, Policar M. Number of oral contraceptive pill packages dispensed and subsequent unintended pregnancies. Obstet Gynecol 2011;117:566-72. http://dx.doi. org/10.1097/AOG.0b013e3182056309
236. Chin-Quee DS, Cuthbertson C, Janowitz B. Over-the-counter pill provision: evidence from Jamaica. Stud Fam Plann 2006;37:99-110. http://dx.doi.org/10.1111/j.1728-4465.2006.00089.x

237. Zapata LB, Steenland MW, Brahmi D, Marchbanks PA, Curtis KM. Effect of missed combined hormonal contraceptives on contraceptive effectiveness: a systematic review. Contraception 2013;87:685-700. http://dx.doi.org/10.1016/j.contraception.2012.08.035

238. Anttila L, Kunz M, Marr J. Bleeding pattern with drospirenone $3 \mathrm{mg}+$ ethinyl estradiol $20 \mathrm{mcg} 24 / 4$ combined oral contraceptive compared with desogestrel 150 mcg+ethinyl estradiol 20 mcg 21/7 combined oral contraceptive. Contraception 2009;80:445-51. http:// dx.doi.org/10.1016/j.contraception.2009.03.013

239. Chowdhury V, Joshi UM, Gopalkrishna K, Betrabet S, Mehta S, Saxena $\mathrm{BN}$. 'Escape' ovulation in women due to the missing of low dose combination oral contraceptive pills. Contraception 1980;22:241-7. http://dx.doi.org/10.1016/S0010-7824(80)80003-5

240. Christin-Maitre S, Serfaty D, Chabbert-Buffet N, Ochsenbein E, Chassard D, Thomas JL. Comparison of a 24-day and a 21-day pill regimen for the novel combined oral contraceptive, nomegestrol acetate and 17b-estradiol (NOMAC/E2): a double-blind, randomized study. Hum Reprod 2011;26:1338-47. http://dx.doi.org/10.1093/humrep/der058

241. Creinin MD, Lippman JS, Eder SE, Godwin AJ, Olson W. The effect of extending the pill-free interval on follicular activity: triphasic norgestimate/35 $\mu \mathrm{g}$ ethinyl estradiol versus monophasic levonorgestrel/20 $\mu \mathrm{g}$ ethinyl estradiol. Contraception 2002;66:147-52. http://dx.doi.org/10.1016/S0010-7824(02)00344-X

242. Dinger J, Minh TD, Buttmann N, Bardenheuer K. Effectiveness of oral contraceptive pills in a large U.S. cohort comparing progestogen and regimen. Obstet Gynecol 2011;117:33-40. http://dx.doi. org/10.1097/AOG.0b013e31820095a2

243. Elomaa K, Lähteenmäki P. Ovulatory potential of preovulatory sized follicles during oral contraceptive treatment. Contraception 1999;60:275-9. http://dx.doi.org/10.1016/S0010-7824(99)00094-3

244. Elomaa K, Rolland R, Brosens I, et al. Omitting the first oral contraceptive pills of the cycle does not automatically lead to ovulation. Am J Obstet Gynecol 1998;179:41-6. http://dx.doi.org/10.1016/ S0002-9378(98)70249-2

245. Endrikat J, Wessel J, Rosenbaum P, Düsterberg B. Plasma concentrations of endogenous hormones during one regular treatment cycle with a low-dose oral contraceptive and during two cycles with deliberate omission of two tablets. Gynecol Endocrinol 2004;18:318-26. http:// dx.doi.org/10.1080/0951359042000199869

246. Hamilton CJ, Hoogland HJ. Longitudinal ultrasonographic study of the ovarian suppressive activity of a low-dose triphasic oral contraceptive during correct and incorrect pill intake. Am J Obstet Gynecol 1989;161:1159-62. http://dx.doi.org/10.1016/0002-9378(89)90655-8

247. Hedon B, Cristol P, Plauchut A, et al. Ovarian consequences of the transient interruption of combined oral contraceptives. Int J Fertil 1992;37:270-6.

248. Killick SR. Ovarian follicles during oral contraceptive cycles: their potential for ovulation. Fertil Steril 1989;52:580-2.

249. Killick SR, Bancroft K, Oelbaum S, Morris J, Elstein M. Extending the duration of the pill-free interval during combined oral contraception. Adv Contracept 1990;6:33-40. http://dx.doi.org/10.1007/BF01849485

250. Klipping C, Duijkers I, Trummer D, Marr J. Suppression of ovarian activity with a drospirenone-containing oral contraceptive in a $24 / 4$ regimen. Contraception 2008;78:16-25. http://dx.doi.org/10.1016/j. contraception.2008.02.019 
251. Klipping C, Marr J. Effects of two combined oral contraceptives containing ethinyl estradiol 20 microg combined with either drospirenone or desogestrel on lipids, hemostatic parameters and carbohydrate metabolism. Contraception 2005;71:409-16. http:// dx.doi.org/10.1016/j.contraception.2004.12.005

252. Landgren BM, Csemiczky G. The effect of follicular growth and luteal function of "missing the pill." A comparison between a monophasic and a triphasic combined oral contraceptive. Contraception 1991;43:149_ 59. http://dx.doi.org/10.1016/0010-7824(91)90042-E

253. Landgren BM, Diczfalusy E. Hormonal consequences of missing the pill during the first two days of three consecutive artificial cycles. Contraception 1984;29:437-46. http://dx.doi.org/10.1016/0010-7824(84)90017-9

254. Letterie GS. A regimen of oral contraceptives restricted to the periovulatory period may permit folliculogenesis but inhibit ovulation. Contraception 1998;57:39-44. http://dx.doi.org/10.1016/ S0010-7824(97)00205-9

255. Letterie GS, Chow GE. Effect of “missed” pills on oral contraceptive effectiveness. Obstet Gynecol 1992;79:979-82.

256. Morris SE, Groom GV, Cameron ED, Buckingham MS, Everitt JM, Elstein M. Studies on low dose oral contraceptives: plasma hormone changes in relation to deliberate pill ('Microgynon 30') omission. Contraception 1979;20:61-9. http://dx.doi. org/10.1016/0010-7824(79)90045-3

257. Nakajima ST, Archer DF, Ellman H. Efficacy and safety of a new 24-day oral contraceptive regimen of norethindrone acetate $1 \mathrm{mg} /$ ethinyl estradiol $20 \mu \mathrm{g}$ (Loestrin $24 \mathrm{Fe}$ ). Contraception 2007;75:16-22. http:// dx.doi.org/10.1016/j.contraception.2006.08.004

258. Nuttall ID, Elstein M, McCafferty E, Seth J, Cameron ED. The effect of ethinyl estradiol $20 \mathrm{mcg}$ and levonorgestrel $250 \mathrm{mcg}$ on the pituitary-ovarian function during normal tablet-taking and when tablets are missed. Contraception 1982;26:121-35. http://dx.doi. org/10.1016/0010-7824(82)90081-6

259. Pierson RA, Archer DF, Moreau M, Shangold GA, Fisher AC, Creasy GW. Ortho Evra/Evra versus oral contraceptives: follicular development and ovulation in normal cycles and after an intentional dosing error. Fertil Steril 2003;80:34-42. http://dx.doi.org/10.1016/ S0015-0282(03)00556-9

260. Rible RD, Taylor D, Wilson ML, Stanczyk FZ, Mishell DR Jr. Follicular development in a 7-day versus 4-day hormone-free interval with an oral contraceptive containing $20 \mathrm{mcg}$ ethinyl estradiol and $1 \mathrm{mg}$ norethindrone acetate. Contraception 2009;79:182-8. http://dx.doi. org/10.1016/j.contraception.2008.10.005

261. Schlaff WD, Lynch AM, Hughes HD, Cedars MI, Smith DL. Manipulation of the pill-free interval in oral contraceptive pill users: the effect on follicular suppression. Am J Obstet Gynecol 2004;190:94351. http://dx.doi.org/10.1016/j.ajog.2004.02.012

262. Spona J, Elstein M, Feichtinger W, et al. Shorter pill-free interval in combined oral contraceptives decreases follicular development. Contraception 1996;54:71-7. http://dx.doi. org/10.1016/0010-7824(96)00137-0

263. Sullivan H, Furniss H, Spona J, Elstein M. Effect of 21-day and 24-day oral contraceptive regimens containing gestodene $(60 \mu \mathrm{g})$ and ethinyl estradiol $(15 \mu \mathrm{g})$ on ovarian activity. Fertil Steril 1999;72:115-20. http://dx.doi.org/10.1016/S0015-0282(99)00205-8

264. Wang E, Shi S, Cekan SZ, Landgren BM, Diczfalusy E. Hormonal consequences of "missing the pill." Contraception 1982;26:545-66. http://dx.doi.org/10.1016/0010-7824(82)90131-7
265. Willis SA, Kuehl TJ, Spiekerman AM, Sulak PJ. Greater inhibition of the pituitary-ovarian axis in oral contraceptive regimens with a shortened hormone-free interval. Contraception 2006;74:100-3. http://dx.doi.org/10.1016/j.contraception.2006.02.006

266. Abrams LS, Skee DM, Natarajan J, et al. Pharmacokinetics of norelgestromin and ethinyl estradiol delivered by a contraceptive patch (Ortho Evra/Evra) under conditions of heat, humidity, and exercise. J Clin Pharmacol 2001;41:1301-9. http://dx.doi. org/10.1177/00912700122012887

267. Ahrendt HJ, Nisand I, Bastianelli C, et al. Efficacy, acceptability and tolerability of the combined contraceptive ring, NuvaRing, compared with an oral contraceptive containing 30 microg of ethinyl estradiol and $3 \mathrm{mg}$ of drospirenone. Contraception 2006;74:451-7. http:// dx.doi.org/10.1016/j.contraception.2006.07.004

268. Bjarnadóttir RI, Tuppurainen M, Killick SR. Comparison of cycle control with a combined contraceptive vaginal ring and oral levonorgestrel/ethinyl estradiol. Am J Obstet Gynecol 2002;186:389_ 95. http://dx.doi.org/10.1067/mob.2002.121103

269. Brucker C, Karck U, Merkle E. Cycle control, tolerability, efficacy and acceptability of the vaginal contraceptive ring, NuvaRing: results of clinical experience in Germany. Eur J Contracept Reprod Health Care 2008;13:31-8. http://dx.doi.org/10.1080/13625180701577122

270. Dieben TO, Roumen FJ, Apter D. Efficacy, cycle control, and user acceptability of a novel combined contraceptive vaginal ring. Obstet Gynecol 2002;100:585-93.

271. Mulders TM, Dieben TO. Use of the novel combined contraceptive vaginal ring NuvaRing for ovulation inhibition. Fertil Steril 2001;75:865-70. http://dx.doi.org/10.1016/S0015-0282(01)01689-2

272. Guilbert E, Boroditsky R, Black A, et al; Society of Obstetricians and Gynaecologists of Canada. Canadian Consensus Guideline on Continuous and Extended Hormonal Contraception, 2007. J Obstet Gynaecol Can 2007;29(Suppl 2):S1-32. http://dx.doi.org/10.1016/ S1701-2163(16)32526-9

273. Wiegratz I, Stahlberg S, Manthey T, et al. Effect of extended-cycle regimen with an oral contraceptive containing $30 \mathrm{mcg}$ ethinylestradiol and $2 \mathrm{mg}$ dienogest on bleeding patterns, safety, acceptance and contraceptive efficacy. Contraception 2011;84:133-43. http://dx.doi. org/10.1016/j.contraception.2011.01.002

274. Hubacher D, Fortney J. Follow-up visits after IUD insertion. Are more better? J Reprod Med 1999;44:801-6.

275. Godfrey EM, Whiteman MK, Curtis KM. Treatment of unscheduled bleeding in women using extended- or continuoususe combined hormonal contraception: a systematic review. Contraception 2013;87:567-75. http://dx.doi.org/10.1016/j. contraception.2012.08.005

276. Sulak PJ, Kuehl TJ, Coffee A, Willis S. Prospective analysis of occurrence and management of breakthrough bleeding during an extended oral contraceptive regimen. Am J Obstet Gynecol 2006;195:935-41. http://dx.doi.org/10.1016/j.ajog.2006.02.048

277. Sulak PJ, Smith V, Coffee A, Witt I, Kuehl AL, Kuehl TJ. Frequency and management of breakthrough bleeding with continuous use of the transvaginal contraceptive ring: a randomized controlled trial. Obstet Gynecol 2008;112:563-71. http://dx.doi.org/10.1097/ AOG.0b013e3181842071

278. Kaneshiro B, Edelman A, Carlson N, Morgan K, Nichols M, Jensen J. Treatment of unscheduled bleeding in continuous oral contraceptive users with doxycycline: a randomized controlled trial. Obstet Gynecol 2010;115:1141-9. http://dx.doi.org/10.1097/ AOG.0b013e3181e 0119c 
279. McCann MF, Potter LS. Progestin-only oral contraception: a comprehensive review. Contraception 1994;50(Suppl 1):S1-195. http://dx.doi.org/10.1016/0010-7824(94)90124-4

280. Arévalo M, Jennings V, Sinai I. Efficacy of a new method of family planning: the Standard Days Method. Contraception 2002;65:333-8. http://dx.doi.org/10.1016/S0010-7824(02)00288-3

281. Arévalo M, Sinai I, Jennings V. A fixed formula to define the fertile window of the menstrual cycle as the basis of a simple method of natural family planning. Contraception 1999;60:357-60. http://dx.doi. org/10.1016/S0010-7824(99)00106-7

282. Wilcox AJ, Dunson DB, Weinberg CR, Trussell J, Baird DD. Likelihood of conception with a single act of intercourse: providing benchmark rates for assessment of post-coital contraceptives. Contraception 2001;63:211-5. http://dx.doi.org/10.1016/S0010-7824(01)00191-3

283. Cleland K, Zhu H, Goldstuck N, Cheng L, Trussell J. The efficacy of intrauterine devices for emergency contraception: a systematic review of 35 years of experience. Hum Reprod 2012;27:1994-2000. http:// dx.doi.org/10.1093/humrep/des140

284. Glasier AF, Cameron ST, Fine PM, et al. Ulipristal acetate versus levonorgestrel for emergency contraception: a randomised noninferiority trial and meta-analysis. Lancet 2010;375:555-62. http:// dx.doi.org/10.1016/S0140-6736(10)60101-8

285. Raymond E, Taylor D, Trussell J, Steiner MJ. Minimum effectiveness of the levonorgestrel regimen of emergency contraception. Contraception 2004;69:79-81. http://dx.doi.org/10.1016/j. contraception.2003.09.013

286. Jatlaoui TC, Curtis KM. Safety and effectiveness data for emergency contraceptive pills among women with obesity: a systematic review. Contraception 2016. Epub May 24, 2016. http://dx.doi.org/10.1016/j. contraception.2016.05.002

287. Fine P, Mathé H, Ginde S, Cullins V, Morfesis J, Gainer E. Ulipristal acetate taken 48-120 hours after intercourse for emergency contraception. Obstet Gynecol 2010;115:257-63. http://dx.doi. org/10.1097/AOG.0b013e3181c8e2aa

288. Dada OA, Godfrey EM, Piaggio G, von Hertzen H; Nigerian Network for Reproductive Health Research and Training. A randomized, doubleblind, noninferiority study to compare two regimens of levonorgestrel for emergency contraception in Nigeria. Contraception 2010;82:3738. http://dx.doi.org/10.1016/j.contraception.2010.06.004

289. Ellertson C, Evans M, Ferden S, et al. Extending the time limit for starting the Yuzpe regimen of emergency contraception to 120 hours. Obstet Gynecol 2003;101:1168-71.

290. Ngai SW, Fan S, Li S, et al. A randomized trial to compare $24 \mathrm{~h}$ versus 12 $\mathrm{h}$ double dose regimen of levonorgestrel for emergency contraception. Hum Reprod 2005;20:307-11. http://dx.doi.org/10.1093/humrep/deh583

291. Rodrigues I, Grou F, Joly J. Effectiveness of emergency contraceptive pills between 72 and 120 hours after unprotected sexual intercourse. Am J Obstet Gynecol 2001;184:531-7. http://dx.doi.org/10.1067/mob.2001.111102

292. von Hertzen H, Piaggio G, Ding J, et al; WHO Research Group on Post-ovulatory Methods of Fertility Regulation. Low dose mifepristone and two regimens of levonorgestrel for emergency contraception: a WHO multicentre randomised trial. Lancet 2002;360:1803-10. http:// dx.doi.org/10.1016/S0140-6736(02)11767-3

293. Piaggio G, Kapp N, von Hertzen H. Effect on pregnancy rates of the delay in the administration of levonorgestrel for emergency contraception: a combined analysis of four WHO trials. Contraception 2011;84:35-9. http://dx.doi.org/10.1016/j.contraception.2010.11.010
294. Rodriguez MI, Curtis KM, Gaffield ML, Jackson E, Kapp N. Advance supply of emergency contraception: a systematic review. Contraception 2013;87:590-601. http://dx.doi.org/10.1016/j. contraception.2012.09.011

295. Brache V, Cochon L, Duijkers IJ, et al. A prospective, randomized, pharmacodynamic study of quick-starting a desogestrel progestin-only pill following ulipristal acetate for emergency contraception. Hum Reprod 2015;30:2785-93.

296. Cameron ST, Berger C, Michie L, Klipping C, Gemzell-Danielsson K. The effects on ovarian activity of ulipristal acetate when 'quickstarting' a combined oral contraceptive pill: a prospective, randomized, doubleblind parallel-arm, placebo-controlled study. Hum Reprod 2015;30:1566-72. http://dx.doi.org/10.1093/humrep/dev115

297. Salcedo J, Rodriguez MI, Curtis KM, Kapp N. When can a woman resume or initiate contraception after taking emergency contraceptive pills? A systematic review. Contraception 2013;87:602-4. http://dx.doi. org/10.1016/j.contraception.2012.08.013

298. Rodriguez MI, Godfrey EM, Warden M, Curtis KM. Prevention and management of nausea and vomiting with emergency contraception: a systematic review. Contraception 2013;87:583-9. http://dx.doi. org/10.1016/j.contraception.2012.09.031

299. Creinin MD, Schlaff W, Archer DF, et al. Progesterone receptor modulator for emergency contraception: a randomized controlled trial. Obstet Gynecol 2006;108:1089-97. http://dx.doi.org/10.1097/01. AOG.0000239440.02284.45

300. Farajkhoda T, Khoshbin A, Enjezab B, Bokaei M, Karimi Zarchi M. Assessment of two emergency contraceptive regimens in Iran: levonorgestrel versus the Yuzpe. Niger J Clin Pract 2009;12:450-2.

301. Ho PC, Kwan MSW. A prospective randomized comparison of levonorgestrel with the Yuzpe regimen in post-coital contraception. Hum Reprod 1993;8:389-92.

302. Arowojolu AO, Okewole IA, Adekunle AO. Comparative evaluation of the effectiveness and safety of two regimens of levonorgestrel for emergency contraception in Nigerians. Contraception 2002;66:26973. http://dx.doi.org/10.1016/S0010-7824(02)00337-2

303. Ragan RE, Rock RW, Buck HW. Metoclopramide pretreatment attenuates emergency contraceptive-associated nausea. Am J Obstet Gynecol 2003;188:330-3. http://dx.doi.org/10.1067/mob.2003.90

304. Raymond EG, Creinin MD, Barnhart KT, Lovvorn AE, Rountree RW, Trussell J. Meclizine for prevention of nausea associated with use of emergency contraceptive pills: a randomized trial. Obstet Gynecol 2000;95:271-7.

305. Peterson HB. Sterilization. Obstet Gynecol 2008;111:189-203. http:// dx.doi.org/10.1097/01.AOG.0000298621.98372.62

306. Peterson HB, Xia Z, Hughes JM, Wilcox LS, Tylor LR, Trussell J. The risk of pregnancy after tubal sterilization: findings from the U.S. Collaborative Review of Sterilization. Am J Obstet Gynecol 1996;174:1161-8, discussion 1168-70. http://dx.doi.org/10.1016/S0002-9378(96)70658-0

307. Lawrie TA, Nardin JM, Kulier R, Boulvain M. Techniques for the interruption of tubal patency for female sterilisation. Cochrane Database Syst Rev 2011; (2):CD003034.

308. Cleary TP, Tepper NK, Cwiak C, et al. Pregnancies after hysteroscopic sterilization: a systematic review. Contraception 2013;87:539-48. http://dx.doi.org/10.1016/j.contraception.2012.08.006

309. Legendre G, Levaillant JM, Faivre E, Deffieux X, Gervaise A, Fernandez H. $3 \mathrm{D}$ ultrasound to assess the position of tubal sterilization microinserts. Hum Reprod 2011;26:2683-9. http://dx.doi.org/10.1093/humrep/der242

310. Levy B, Levie MD, Childers ME. A summary of reported pregnancies after hysteroscopic sterilization. J Minim Invasive Gynecol 2007;14:271-4. http://dx.doi.org/10.1016/j.jmig.2006.11.007 
311. Veersema S, Vleugels MP, Moolenaar LM, Janssen CA, Brölmann HA. Unintended pregnancies after Essure sterilization in the Netherlands. Fertil Steril 2010;93:35-8. http://dx.doi.org/10.1016/j.fertnstert.2008.10.005

312. Arjona JE, Miño M, Cordón J, Povedano B, Pelegrin B, Castelo-Branco C. Satisfaction and tolerance with office hysteroscopic tubal sterilization. Fertil Steril 2008;90:1182-6. http://dx.doi.org/10.1016/j.fertnstert.2007.08.007

313. Duffy S, Marsh F, Rogerson L, et al. Female sterilisation: a cohort controlled comparative study of ESSURE versus laparoscopic sterilisation. BJOG 2005;112:1522-8. http://dx.doi.org/10.1111/j.1471-0528.2005.00726.x

314. Grosdemouge I, Engrand JB, Dhainault C, et al. Essure implants for tubal sterilisation in France [French]. Gynecol Obstet Fertil 2009;37:389-95. http://dx.doi.org/10.1016/j.gyobfe.2009.03.024

315. Levie MD, Chudnoff SG. Prospective analysis of office-based hysteroscopic sterilization. J Minim Invasive Gynecol 2006;13:98-101. http://dx.doi.org/10.1016/j.jmig.2005.11.010

316. Shavell VI, Abdallah ME, Diamond MP, Berman JM. Placement of a permanent birth control device at a university medical center. J Reprod Med 2009;54:218-22.

317. Teisala K. Removal of an intrauterine device and the treatment of acute pelvic inflammatory disease. Ann Med 1989;21:63-5. http://dx.doi. org/10.3109/07853898909149184

318. Bedford JM, Zelikovsky G. Viability of spermatozoa in the human ejaculate after vasectomy. Fertil Steril 1979;32:460-3.

319. Edwards IS. Earlier testing after vasectomy, based on the absence of motile sperm. Fertil Steril 1993;59:431-6.

320. Jouannet P, David G. Evolution of the properties of semen immediately following vasectomy. Fertil Steril 1978;29:435-41.

321. Labrecque M, Hays M, Chen-Mok M, Barone MA, Sokal D. Frequency and patterns of early recanalization after vasectomy. BMC Urol 2006;6:25. http://dx.doi.org/10.1186/1471-2490-6-25

322. Alderman PM. The lurking sperm. A review of failures in 8879 vasectomies performed by one physician. JAMA 1988;259:3142-4. http://dx.doi.org/10.1001/jama.1988.03720210032024

323. Black T, Francome C. The evolution of the Marie Stopes electrocautery no-scalpel vasectomy procedure. J Fam Plann Reprod Health Care 2002;28:137-8. http://dx.doi.org/10.1783/147118902101196270

324. Davies AH, Sharp RJ, Cranston D, Mitchell RG. The long-term outcome following "special clearance" after vasectomy. Br J Urol 1990;66:211-2. http://dx.doi.org/10.1111/j.1464-410X.1990.tb14907.x

325. Philp T, Guillebaud J, Budd D. Late failure of vasectomy after two documented analyses showing azoospermic semen. Br Med J (Clin Res Ed) 1984;289:77-9. http://dx.doi.org/10.1136/bmj.289.6437.77

326. Philp T, Guillebaud J, Budd D. Complications of vasectomy: review of 16,000 patients. Br J Urol 1984;56:745-8. http://dx.doi. org/10.1111/j.1464-410X.1984.tb06161.x

327. Belker AM, Sexter MS, Sweitzer SJ, Raff MJ. The high rate of noncompliance for post-vasectomy semen examination: medical and legal considerations. J Urol 1990;144:284-6.

328. Chawla A, Bowles B, Zini A. Vasectomy follow-up: clinical significance of rare nonmotile sperm in postoperative semen analysis. Urology 2004;64:1212-5. http://dx.doi.org/10.1016/j.urology.2004.07.007

329. Labrecque M, Bédard L, Laperrière L. Efficacy and complications associated with vasectomies in two clinics in the Quebec region [French]. Can Fam Physician 1998;44:1860-6.

330. Labrecque M, Nazerali H, Mondor M, Fortin V, Nasution M. Effectiveness and complications associated with 2 vasectomy occlusion techniques. J Urol 2002;168:2495-8, discussion 2498. http://dx.doi. org/10.1016/S0022-5347(05)64176-6
331. Maatman TJ, Aldrin L, Carothers GG. Patient noncompliance after vasectomy. Fertil Steril 1997;68:552-5. http://dx.doi.org/10.1016/ S0015-0282(97)00251-3

332. Dhar NB, Jones JS, Bhatt A, Babineau D. A prospective evaluation of the impact of scheduled follow-up appointments with compliance rates after vasectomy. BJU Int 2007;99:1094-7. http://dx.doi. org/10.1111/j.1464-410X.2006.06725.x

333. Hillard PJ, Berek JS, Barss VA, et al. Guidelines for women's health care: A resource manual. 3rd ed. Washington, DC: American College of Obstetricians and Gynecologists; 2007.

334. Shifren J, Gass M; NAMS Recommendations for Clinical Care of Midlife Women Working Group. The North American Menopause Society recommendation for clinical care of midlife women. Menopause 2014;21;1038-62.

335. te Velde ER, Pearson PL. The variability of female reproductive ageing. Hum Reprod Update 2002;8:141-54. http://dx.doi.org/10.1093/humupd/8.2.141

336. Broekmans FJ, Soules MR, Fauser BC. Ovarian aging: mechanisms and clinical consequences. Endocr Rev 2009;30:465-93. http://dx.doi. org/10.1210/er.2009-0006

337. Wood JW. Fecundity and natural fertility in humans. Oxf Rev Reprod Biol 1989;11:61-109.

338. Balasch J, Gratacós E. Delayed childbearing: effects on fertility and the outcome of pregnancy. Curr Opin Obstet Gynecol 2012;24:187-93. http://dx.doi.org/10.1097/GCO.0b013e3283517908

339. Bateman BT, Simpson LL. Higher rate of stillbirth at the extremes of reproductive age: a large nationwide sample of deliveries in the United States. Am J Obstet Gynecol 2006;194:840-5. http://dx.doi. org/10.1016/j.ajog.2005.08.038

340. Berg CJ, Callaghan WM, Syverson C, Henderson Z. Pregnancyrelated mortality in the United States, 1998 to 2005. Obstet Gynecol 2010;116:1302-9. http://dx.doi.org/10.1097/AOG.0b013e3181fdfb11

341. Lidegaard $\varnothing$, Løkkegaard E, Svendsen AL, Agger C. Hormonal contraception and risk of venous thromboembolism: national follow-up study. BMJ 2009;339:b2890.

342. Lidegaard $\varnothing$, Nielsen LH, Skovlund CW, Skjeldestad FE, Løkkegaard E. Risk of venous thromboembolism from use of oral contraceptives containing different progestogens and oestrogen doses: Danish cohort study, 2001-9. BMJ 2011;343:d6423.

343. Nightingale AL, Lawrenson RA, Simpson EL, Williams TJ, MacRae KD, Farmer RD. The effects of age, body mass index, smoking and general health on the risk of venous thromboembolism in users of combined oral contraceptives. Eur J Contracept Reprod Health Care 2000;5:265-74. http://dx.doi.org/10.1080/13625180008500402

344. Slone D, Shapiro S, Kaufman DW, Rosenberg L, Miettinen OS, Stolley PD. Risk of myocardial infarction in relation to current and discontinued use of oral contraceptives. N Engl J Med 1981;305:420-4. http://dx.doi.org/10.1056/NEJM198108203050802

345. Tanis BC, van den Bosch MA, Kemmeren JM, et al. Oral contraceptives and the risk of myocardial infarction. N Engl J Med 2001;345:178793. http://dx.doi.org/10.1056/NEJMoa003216

346. Collaborative Group on Hormonal Factors in Breast Cancer. Breast cancer and hormonal contraceptives: collaborative reanalysis of individual data on 53297 women with breast cancer and 100239 women without breast cancer from 54 epidemiological studies. Lancet 1996;347:1713-27. http://dx.doi.org/10.1016/S0140-6736(96)90806-5

347. Gill JK, Press MF, Patel AV, Bernstein L. Oral contraceptive use and risk of breast carcinoma in situ (United States). Cancer Causes Control 2006;17:1155-62. http://dx.doi.org/10.1007/s10552-006-0056-0 
348. Kumle M, Weiderpass E, Braaten T, Persson I, Adami HO, Lund E. Use of oral contraceptives and breast cancer risk: The NorwegianSwedish Women's Lifestyle and Health Cohort Study. Cancer Epidemiol Biomarkers Prev 2002;11:1375-81.

349. Marchbanks PA, McDonald JA, Wilson HG, et al. Oral contraceptives and the risk of breast cancer. N Engl J Med 2002;346:2025-32. http:// dx.doi.org/10.1056/NEJMoa013202

350. Newcomb PA, Longnecker MP, Storer BE, et al. Recent oral contraceptive use and risk of breast cancer (United States). Cancer Causes Control 1996;7:525-32. http://dx.doi.org/10.1007/BF00051885
351. Rosenberg L, Palmer JR, Rao RS, et al. Case-control study of oral contraceptive use and risk of breast cancer. Am J Epidemiol 1996;143:2537. http://dx.doi.org/10.1093/oxfordjournals.aje.a008654

352. Rosenberg L, Zhang Y, Coogan PF, Strom BL, Palmer JR. A case-control study of oral contraceptive use and incident breast cancer. Am J Epidemiol 2009;169:473-9. http://dx.doi.org/10.1093/aje/kwn360

353. Shapiro S, Rosenberg L, Hoffman M, et al. Risk of breast cancer in relation to the use of injectable progestogen contraceptives and combined estrogen/progestogen contraceptives. Am J Epidemiol 2000;151:396403. http://dx.doi.org/10.1093/oxfordjournals.aje.a010219 


\section{Appendix A}

\section{Summary Chart of U.S. Medical Eligibility Criteria for Contraceptive Use, 2016}

Health-care providers can use the summary table as a quick reference guide to the classifications for hormonal contraceptive methods and intrauterine contraception to compare classifications across these methods (Box A1) (Table A1). For

\section{BOX A1. Categories for classifying hormonal contraceptives and} intrauterine devices

$1=$ A condition for which there is no restriction for the use of the contraceptive method.

$2=$ A condition for which the advantages of using the method generally outweigh the theoretical or proven risks.

$3=$ A condition for which the theoretical or proven risks usually outweigh the advantages of using the method.

$4=$ A condition that represents an unacceptable health risk if the contraceptive method is used. complete guidance, see the 2016 U.S. Medical Eligibility Criteria for Contraceptive Use (U.S. MEC) (Curtis KM, Tepper NK, Jatlaoui TC, et al. U.S. medical eligibility criteria for contraceptive use, 2016. MMWR Recomm Rep 2016;65[No. RR-3]) for clarifications to the numeric categories, as well as for summaries of the evidence and additional comments. Hormonal contraceptives and intrauterine devices do not protect against sexually transmitted diseases (STDs), including human immunodeficiency virus (HIV), and women using these methods should be counseled that consistent and correct use of the male latex condom reduces the risk for transmission of HIV and other STDs. Use of female condoms can provide protection from transmission of STDs, although data are limited.

TABLE A1. Summary of classifications for hormonal contraceptive methods and intrauterine devices

\begin{tabular}{|c|c|c|c|c|c|c|}
\hline Condition & Cu-IUD & LNG-IUD & Implants & DMPA & POP & CHCs \\
\hline \multicolumn{7}{|c|}{ Personal Characteristics and Reproductive History } \\
\hline Pregnancy & $4^{*}$ & $4^{*}$ & $N A^{*}$ & $N A^{*}$ & $N A^{*}$ & $N A^{*}$ \\
\hline \multirow[t]{2}{*}{ Age } & $\begin{array}{l}\text { Menarche to } \\
<20 \text { years: } 2\end{array}$ & $\begin{array}{l}\text { Menarche to } \\
<20 \text { years: } 2\end{array}$ & $\begin{array}{l}\text { Menarche to } \\
<18 \text { years: } 1\end{array}$ & $\begin{array}{l}\text { Menarche to } \\
<18 \text { years: } 2\end{array}$ & $\begin{array}{l}\text { Menarche to } \\
<18 \text { years: } 1\end{array}$ & $\begin{array}{l}\text { Menarche to } \\
<40 \text { years: } 1\end{array}$ \\
\hline & $\geq 20$ years: 1 & $\geq 20$ years: 1 & $\begin{array}{l}\text { 18-45 years: } 1 \\
\text { >45 years: } 1\end{array}$ & $\begin{array}{l}\text { 18-45 years: } 1 \\
\text { >45 years: } 2\end{array}$ & $\begin{array}{l}\text { 18-45 years: } 1 \\
\text { >45 years: } 1\end{array}$ & $\geq 40$ years: 2 \\
\hline \multicolumn{7}{|l|}{ Parity } \\
\hline a. Nulliparous & 2 & 2 & 1 & 1 & 1 & 1 \\
\hline b. Parous & 1 & 1 & 1 & 1 & 1 & 1 \\
\hline \multicolumn{7}{|l|}{ Breastfeeding } \\
\hline $\begin{array}{l}\text { a. }<21 \text { days postpartum } \\
\text { b. } 21 \text { to }<30 \text { days postpartum }\end{array}$ & - & - & $2^{*}$ & $2^{*}$ & $2^{*}$ & $4^{*}$ \\
\hline $\begin{array}{l}\text { i. With other risk factors for } \\
\text { VTE (e.g., age } \geq 35 \text { years, } \\
\text { previous VTE, thrombo- } \\
\text { philia, immobility, } \\
\text { transfusion at delivery, } \\
\text { peripartum cardiomyopa- } \\
\text { thy, BMI } \geq 30 \mathrm{~kg} / \mathrm{m}^{2} \text {, } \\
\text { postpartum hemorrhage, } \\
\text { postcesarean delivery, } \\
\text { preeclampsia, or smoking) }\end{array}$ & - & - & $2^{*}$ & $2^{*}$ & $2^{*}$ & $3^{*}$ \\
\hline $\begin{array}{l}\text { ii. Without other risk factors } \\
\text { for VTE }\end{array}$ & - & - & $2^{*}$ & $2^{*}$ & $2^{*}$ & $3^{*}$ \\
\hline \multicolumn{7}{|l|}{ c. $30-42$ days postpartum } \\
\hline $\begin{array}{l}\text { i. With other risk factors for } \\
\text { VTE (e.g., age } \geq 35 \text { years, } \\
\text { previous VTE, thrombo- } \\
\text { philia, immobility, } \\
\text { transfusion at delivery, } \\
\text { peripartum cardiomyopa- } \\
\text { thy, BMI } \geq 30 \mathrm{~kg} / \mathrm{m}^{2} \text {, } \\
\text { postpartum hemorrhage, } \\
\text { postcesarean delivery, } \\
\text { preeclampsia, or smoking) }\end{array}$ & - & - & $1^{*}$ & $1^{*}$ & $1^{*}$ & $3^{*}$ \\
\hline
\end{tabular}

See table footnotes on page 61. 
TABLE A1. (Continued) Summary of classifications for hormonal contraceptive methods and intrauterine devices

\begin{tabular}{|c|c|c|c|c|c|c|}
\hline Condition & Cu-IUD & LNG-IUD & Implants & DMPA & POP & CHCs \\
\hline $\begin{array}{l}\text { ii. Without other risk factors } \\
\text { for VTE }\end{array}$ & - & - & $1^{*}$ & $1^{*}$ & $1^{*}$ & $2^{*}$ \\
\hline d. $>42$ days postpartum & - & - & $1^{*}$ & $1^{*}$ & $1^{*}$ & $2^{*}$ \\
\hline \multicolumn{7}{|l|}{$\begin{array}{l}\text { Postpartum } \\
\text { (nonbreastfeeding women) }\end{array}$} \\
\hline $\begin{array}{l}\text { a. }<21 \text { days postpartum } \\
\text { b. } 21-42 \text { days postpartum }\end{array}$ & - & - & 1 & 1 & 1 & 4 \\
\hline $\begin{array}{l}\text { i. With other risk factors for } \\
\text { VTE (e.g., age } \geq 35 \text { years, } \\
\text { previous VTE, thrombo- } \\
\text { philia, immobility, } \\
\text { transfusion at delivery, } \\
\text { peripartum cardiomyopa- } \\
\text { thy, BMI } \geq 30 \mathrm{~kg} / \mathrm{m}^{2} \text {, } \\
\text { postpartum hemorrhage, } \\
\text { postcesarean delivery, } \\
\text { preeclampsia, or smoking) }\end{array}$ & - & - & 1 & 1 & 1 & $3^{*}$ \\
\hline $\begin{array}{l}\text { ii. Without other risk factors } \\
\text { for VTE }\end{array}$ & - & - & 1 & 1 & 1 & 2 \\
\hline c. $>42$ days postpartum & - & - & 1 & 1 & 1 & 1 \\
\hline \multicolumn{5}{|l|}{$\begin{array}{l}\text { Postpartum (including } \\
\text { cesarean delivery) }\end{array}$} & \multicolumn{2}{|c|}{$\begin{array}{l}\text { a. }<10 \text { minutes after delivery } \\
\text { of the placenta }\end{array}$} \\
\hline i. Breastfeeding & $1^{*}$ & $2^{*}$ & - & - & - & - \\
\hline ii. Nonbreastfeeding & $1^{*}$ & $1^{*}$ & - & - & - & - \\
\hline $\begin{array}{l}\text { b. } 10 \text { minutes after delivery } \\
\text { of the placenta to }<4 \text { weeks } \\
\text { (breastfeeding or } \\
\text { nonbreastfeeding) }\end{array}$ & $2^{*}$ & $2^{*}$ & - & - & - & - \\
\hline $\begin{array}{l}\text { c. } \geq 4 \text { weeks (breastfeeding or } \\
\text { nonbreastfeeding) }\end{array}$ & $1^{*}$ & $1^{*}$ & - & - & - & - \\
\hline d. Postpartum sepsis & 4 & 4 & - & - & - & - \\
\hline \multicolumn{7}{|l|}{ Postabortion } \\
\hline a. First trimester & $1 *$ & $1 *$ & $1 *$ & $1^{*}$ & $1^{*}$ & $1^{*}$ \\
\hline b. Second trimester & $2^{*}$ & $2^{*}$ & $1^{*}$ & $1^{*}$ & $1^{*}$ & $1^{*}$ \\
\hline $\begin{array}{l}\text { c. Immediate postseptic } \\
\text { abortion }\end{array}$ & 4 & 4 & $1^{*}$ & $1^{*}$ & $1^{*}$ & $1^{*}$ \\
\hline Past ectopic pregnancy & 1 & 1 & 1 & 1 & 2 & 1 \\
\hline $\begin{array}{l}\text { History of pelvic surgery (see } \\
\text { Postpartum [Including } \\
\text { Cesarean Delivery] section) }\end{array}$ & 1 & 1 & 1 & 1 & 1 & 1 \\
\hline \multicolumn{7}{|l|}{ Smoking } \\
\hline \multicolumn{7}{|l|}{ b. Age $\geq 35$ years } \\
\hline i. $<15$ cigarettes/day & 1 & 1 & 1 & 1 & 1 & 3 \\
\hline ii. $\geq 15$ cigarettes/day & 1 & 1 & 1 & 1 & 1 & 4 \\
\hline \multicolumn{7}{|l|}{ Obesity } \\
\hline a. $\mathrm{BMI} \geq 30 \mathrm{~kg} / \mathrm{m}^{2}$ & 1 & 1 & 1 & 1 & 1 & 2 \\
\hline $\begin{array}{l}\text { b. Menarche to }<18 \text { years } \\
\text { and } \mathrm{BMI} \geq 30 \mathrm{~kg} / \mathrm{m}^{2}\end{array}$ & 1 & 1 & 1 & 2 & 1 & 2 \\
\hline \multicolumn{7}{|l|}{$\begin{array}{l}\text { This condition is associated } \\
\text { with increased risk for adverse } \\
\text { health events as a result of } \\
\text { pregnancy. }\end{array}$} \\
\hline $\begin{array}{l}\text { a. Restrictive procedures: } \\
\text { decrease storage capacity of } \\
\text { the stomach (vertical banded } \\
\text { gastroplasty, laparoscopic } \\
\text { adjustable gastric band, or } \\
\text { laparoscopic sleeve } \\
\text { gastrectomy) }\end{array}$ & 1 & 1 & 1 & 1 & 1 & 1 \\
\hline $\begin{array}{l}\text { b. Malabsorptive procedures: } \\
\text { decrease absorption of } \\
\text { nutrients and calories by } \\
\text { shortening the functional } \\
\text { length of the small intestine } \\
\text { (Roux-en-Y gastric bypass or } \\
\text { biliopancreatic diversion) }\end{array}$ & 1 & 1 & 1 & 1 & 3 & $\begin{array}{r}\text { COCs: } 3 \\
\text { Patch and ring: } 1\end{array}$ \\
\hline
\end{tabular}

See table footnotes on page 61 . 
TABLE A1. (Continued) Summary of classifications for hormonal contraceptive methods and intrauterine devices

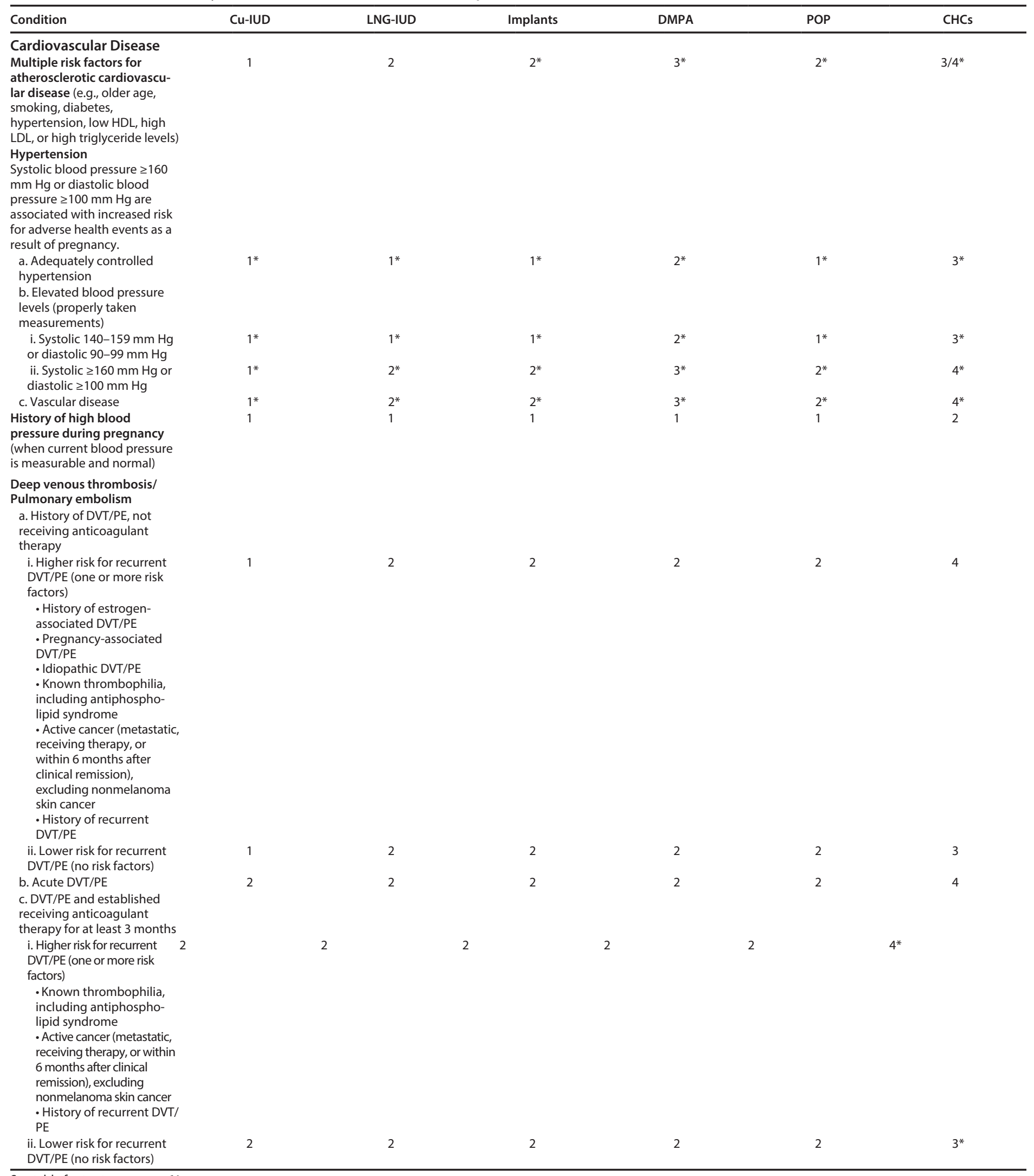

See table footnotes on page 61. 
TABLE A1. (Continued) Summary of classifications for hormonal contraceptive methods and intrauterine devices

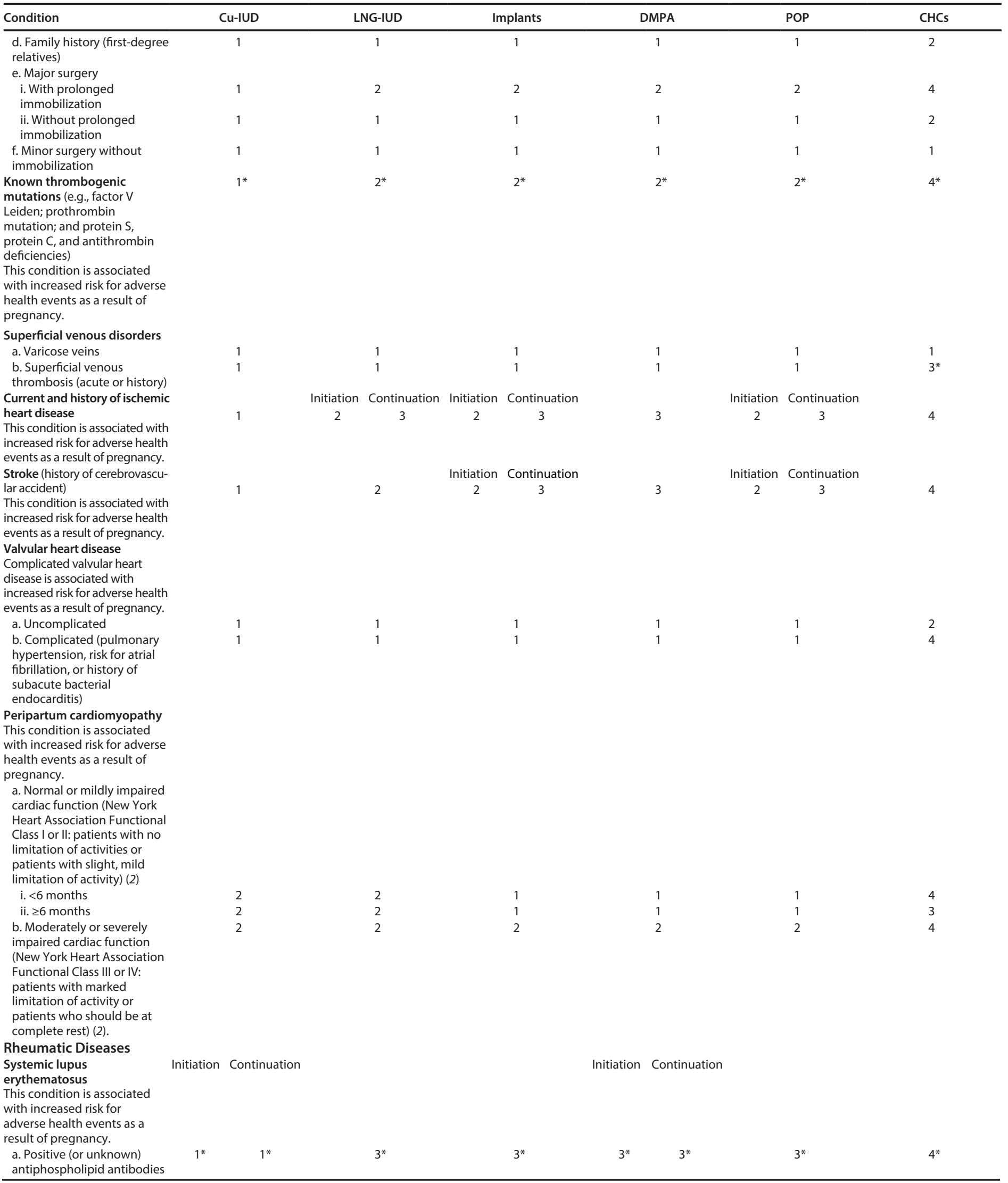

See table footnotes on page 61 . 
TABLE A1. (Continued) Summary of classifications for hormonal contraceptive methods and intrauterine devices

\begin{tabular}{|c|c|c|c|c|c|c|c|c|c|}
\hline \multirow{4}{*}{$\begin{array}{l}\text { Condition } \\
\text { b. Severe thrombocytopenia } \\
\text { c. Immunosuppressive therapy } \\
\text { d. None of the above }\end{array}$} & \multicolumn{2}{|c|}{ Cu-IUD } & \multicolumn{2}{|c|}{ LNG-IUD } & \multirow{2}{*}{$\frac{\text { Implants }}{2^{*}}$} & \multicolumn{2}{|c|}{ DMPA } & \multirow{2}{*}{$\frac{\text { POP }}{2^{*}}$} & \multirow{2}{*}{$\frac{\mathrm{CHCs}}{2^{*}}$} \\
\hline & $3^{*}$ & $2^{*}$ & & $2^{*}$ & & $3^{*}$ & $2^{*}$ & & \\
\hline & $2^{*}$ & $1^{*}$ & & $2^{*}$ & $2^{*}$ & $2^{*}$ & $2^{*}$ & $2^{*}$ & $2^{*}$ \\
\hline & $1^{*}$ & $1^{*}$ & & $2^{*}$ & $2^{*}$ & $2^{*}$ & $2^{*}$ & $2^{*}$ & $2^{*}$ \\
\hline Rheumatoid arthritis & Initiation & Continuation & Initiation & Continuation & & & & & \\
\hline $\begin{array}{l}\text { a. Receiving immunosup- } \\
\text { pressive therapy }\end{array}$ & 2 & 1 & 2 & 1 & 1 & & & 1 & 2 \\
\hline $\begin{array}{l}\text { b. Not receiving immunosup } \\
\text { pressive therapy }\end{array}$ & & 1 & & 1 & 1 & & & 1 & 2 \\
\hline \multicolumn{10}{|l|}{$\begin{array}{l}\text { Neurologic Conditions } \\
\text { Headaches }\end{array}$} \\
\hline $\begin{array}{l}\text { a. Nonmigraine (mild or } \\
\text { severe) } \\
\text { b. Migraine }\end{array}$ & & 1 & & 1 & 1 & & & 1 & $1^{*}$ \\
\hline $\begin{array}{l}\text { i. Without aura (This } \\
\text { category of migraine } \\
\text { includes menstrual } \\
\text { migraine.) }\end{array}$ & & 1 & & 1 & 1 & & & 1 & $2^{*}$ \\
\hline ii. With aura & & 1 & & 1 & 1 & & & 1 & $4^{*}$ \\
\hline $\begin{array}{l}\text { Epilepsy } \\
\text { This condition is associated } \\
\text { with increased risk for adverse } \\
\text { health events as a result of } \\
\text { pregnancy. } \\
\text { Multiple sclerosis }\end{array}$ & & 1 & & 1 & $1^{*}$ & & & $1^{*}$ & $1^{*}$ \\
\hline $\begin{array}{l}\text { a. With prolonged } \\
\text { immobility }\end{array}$ & & 1 & & 1 & 1 & & & 1 & 3 \\
\hline $\begin{array}{l}\text { b. Without prolonged } \\
\text { immobility }\end{array}$ & & 1 & & 1 & 1 & & & 1 & 1 \\
\hline $\begin{array}{l}\text { Depressive Disorders } \\
\text { Depressive disorders }\end{array}$ & & $1^{*}$ & & $1^{*}$ & $1^{*}$ & & & $1^{*}$ & $1^{*}$ \\
\hline \multicolumn{10}{|c|}{ Reproductive Tract Infections and Disorders } \\
\hline $\begin{array}{l}\text { a. Irregular pattern without } \\
\text { heavy bleeding }\end{array}$ & & 1 & 1 & 1 & 2 & & & 2 & 1 \\
\hline $\begin{array}{l}\text { b. Heavy or prolonged } \\
\text { bleeding (includes regular } \\
\text { and irregular patterns) }\end{array}$ & & $2^{*}$ & $1^{*}$ & $2^{*}$ & $2^{*}$ & & & $2^{*}$ & $1^{*}$ \\
\hline $\begin{array}{l}\text { Unexplained vaginal bleeding } \\
\text { (suspicious for serious condition) } \\
\text { before evaluation }\end{array}$ & $\begin{array}{l}\text { Initiation } \\
4^{*}\end{array}$ & $\begin{array}{c}\text { Continuation } \\
2^{*}\end{array}$ & $\begin{array}{c}\text { Initiation } \\
4^{*}\end{array}$ & $\begin{array}{c}\text { Continuation } \\
2^{*}\end{array}$ & $3^{*}$ & & & $2^{*}$ & $2^{*}$ \\
\hline Endometriosis & & 2 & & 1 & 1 & & & 1 & 1 \\
\hline $\begin{array}{l}\text { Benign ovarian tumors } \\
\text { (including cysts) }\end{array}$ & & 1 & & 1 & 1 & & & 1 & 1 \\
\hline Severe dysmenorrhea & & 2 & & 1 & 1 & & & 1 & 1 \\
\hline \multicolumn{10}{|l|}{$\begin{array}{l}\text { Gestational trophoblastic } \\
\text { disease } \\
\text { This condition is associated with } \\
\text { increased risk for adverse health } \\
\text { events as a result of pregnancy. } \\
\text { a. Suspected gestational } \\
\text { trophoblastic disease } \\
\text { (immediate postevacuation) }\end{array}$} \\
\hline i. Uterine size first trimester & & $1^{*}$ & & $1^{*}$ & $1^{*}$ & & & $1^{*}$ & $1^{*}$ \\
\hline $\begin{array}{l}\text { ii. Uterine size second } \\
\text { trimester }\end{array}$ & & $2^{*}$ & & $2^{*}$ & $1^{*}$ & & & $1^{*}$ & $1^{*}$ \\
\hline $\begin{array}{l}\text { b. Confirmed gestational } \\
\text { trophoblastic disease (after } \\
\text { initial evacuation and during } \\
\text { monitoring) }\end{array}$ & Initiation & Continuation & Initiation & Continuation & & & & & \\
\hline $\begin{array}{l}\text { i. Undetectable/ } \\
\text { nonpregnant } \beta \text {-hCG levels }\end{array}$ & $1^{*}$ & $1^{*}$ & $1^{*}$ & $1^{*}$ & $1^{*}$ & & & $1^{*}$ & $1^{*}$ \\
\hline ii. Decreasing $\beta$-hCG levels & $2^{*}$ & $1^{*}$ & $2^{*}$ & $1^{*}$ & $1^{*}$ & & & $1^{*}$ & $1^{*}$ \\
\hline $\begin{array}{l}\text { iii. Persistently elevated } \\
\beta \text {-hCG levels or malignant } \\
\text { disease, with no evidence } \\
\text { or suspicion of intrauterine } \\
\text { disease }\end{array}$ & $2^{*}$ & $1^{*}$ & $2^{*}$ & $1^{*}$ & $1^{*}$ & & & $1^{*}$ & $1^{*}$ \\
\hline
\end{tabular}

See table footnotes on page 61. 
TABLE A1. (Continued) Summary of classifications for hormonal contraceptive methods and intrauterine devices

\begin{tabular}{|c|c|c|c|c|c|c|c|c|}
\hline \multirow{2}{*}{$\begin{array}{l}\text { Condition } \\
\text { iv. Persistently elevated } \\
\beta \text {-hCG levels or malignant } \\
\text { disease, with evidence or } \\
\text { suspicion of intrauterine } \\
\text { disease }\end{array}$} & \multicolumn{2}{|c|}{ CU-IUD } & \multicolumn{2}{|c|}{ LNG-IUD } & \multirow{2}{*}{$\frac{\text { Implants }}{1^{*}}$} & \multirow{2}{*}{$\frac{\text { DMPA }}{1^{*}}$} & \multirow{2}{*}{$\frac{\mathrm{POP}}{1^{*}}$} & \multirow{2}{*}{$\frac{\mathrm{CHCs}}{1^{*}}$} \\
\hline & $4^{*}$ & $2^{*}$ & $4^{*}$ & $2^{*}$ & & & & \\
\hline Cervical ectropion & & 1 & & 1 & 1 & 1 & 1 & 1 \\
\hline $\begin{array}{l}\text { Cervical intraepithelial } \\
\text { neoplasia }\end{array}$ & & 1 & & 2 & 2 & 2 & 1 & 2 \\
\hline Cervical cancer (awaiting & Initiation & Continuation & Initiation & Continuation & & & & \\
\hline treatment) & 4 & 2 & 4 & 2 & 2 & 2 & 1 & 2 \\
\hline \multicolumn{9}{|l|}{$\begin{array}{l}\text { Breast disease } \\
\text { Breast cancer is associated } \\
\text { with increased risk of adverse } \\
\text { health events as a result of } \\
\text { pregnancy. }\end{array}$} \\
\hline a. Undiagnosed mass & & 1 & & 2 & $2^{*}$ & $2^{*}$ & $2^{*}$ & $2^{*}$ \\
\hline b. Benign breast disease & & 1 & & 1 & 1 & 1 & 1 & 1 \\
\hline $\begin{array}{l}\text { c. Family history of cancer } \\
\text { d. Breast cancer }\end{array}$ & & 1 & & 1 & 1 & 1 & 1 & 1 \\
\hline i. Current & & 1 & & 4 & 4 & 4 & 4 & 4 \\
\hline $\begin{array}{l}\text { ii. Past and no evidence of } \\
\text { current disease for } 5 \text { years }\end{array}$ & & 1 & & 3 & 3 & 3 & 3 & 3 \\
\hline Endometrial hyperplasia & & 1 & & 1 & 1 & 1 & 1 & 1 \\
\hline Endometrial cancer & Initiation & Continuation & Initiation & Continuation & & & & \\
\hline $\begin{array}{l}\text { This condition is associated with } \\
\text { increased risk for adverse health } \\
\text { events as a result of pregnancy. }\end{array}$ & 4 & 2 & 4 & 2 & 1 & 1 & 1 & 1 \\
\hline $\begin{array}{l}\text { Ovarian cancer } \\
\text { This condition is associated with } \\
\text { increased risk for adverse health } \\
\text { events as a result of pregnancy. }\end{array}$ & & 1 & & 1 & 1 & 1 & 1 & 1 \\
\hline Uterine fibroids & & 2 & & 2 & 1 & 1 & 1 & 1 \\
\hline \multicolumn{9}{|l|}{ Anatomical abnormalities } \\
\hline $\begin{array}{l}\text { a. Distorted uterine cavity (any } \\
\text { congenital or acquired uterine } \\
\text { abnormality distorting the } \\
\text { uterine cavity in a manner that } \\
\text { is incompatible with } \\
\text { IUD insertion) }\end{array}$ & & 4 & & 4 & - & - & - & - \\
\hline $\begin{array}{l}\text { b. Other abnormalities } \\
\text { (including cervical stenosis or } \\
\text { cervical lacerations) not } \\
\text { distorting the uterine cavity or } \\
\text { interfering with IUD insertion }\end{array}$ & & 2 & & 2 & - & - & - & - \\
\hline \multicolumn{9}{|l|}{ Pelvic inflammatory disease } \\
\hline $\begin{array}{l}\text { a. Past PID (assuming no } \\
\text { current risk factors for STDs) }\end{array}$ & Initiation & Continuation & Initiation & Continuation & & & & \\
\hline $\begin{array}{l}\text { i. With subsequent } \\
\text { pregnancy }\end{array}$ & 1 & 1 & 1 & 1 & 1 & 1 & 1 & 1 \\
\hline $\begin{array}{l}\text { ii. Without subsequent } \\
\text { pregnancy }\end{array}$ & 2 & 2 & 2 & 2 & 1 & 1 & 1 & 1 \\
\hline b. Current PID & 4 & $2^{*}$ & 4 & $2^{*}$ & 1 & 1 & 1 & 1 \\
\hline Sexually transmitted diseases & Initiation & Continuation & Initiation & Continuation & & & & \\
\hline $\begin{array}{l}\text { a. Current purulent cervicitis } \\
\text { or chlamydial infection or } \\
\text { gonococcal infection }\end{array}$ & 4 & $2^{*}$ & 4 & $2^{*}$ & 1 & 1 & 1 & 1 \\
\hline $\begin{array}{l}\text { b. Vaginitis (including } \\
\text { Trichomonas vaginalis and } \\
\text { bacterial vaginosis) }\end{array}$ & 2 & 2 & 2 & 2 & 1 & 1 & 1 & 1 \\
\hline c. Other factors related to STDs & $s \quad 2^{*}$ & 2 & $2^{*}$ & 2 & 1 & 1 & 1 & 1 \\
\hline \multicolumn{9}{|l|}{ HIV } \\
\hline & Initiation & Continuation & Initiation & Continuation & & & & \\
\hline High risk for HIV & 2 & 2 & 2 & 2 & 1 & $1^{*}$ & 1 & 1 \\
\hline $\begin{array}{l}\text { HIV infection } \\
\text { For women with HIV infection } \\
\text { who are not clinically well or not } \\
\text { receiving ARV therapy, this } \\
\text { condition is associated with } \\
\text { increased risk for adverse health } \\
\text { events as a result of pregnancy. }\end{array}$ & - & - & - & - & $1^{*}$ & $1^{*}$ & $1^{*}$ & $1^{*}$ \\
\hline $\begin{array}{l}\text { a. Clinically well receiving } \\
\text { ARV therapy }\end{array}$ & 1 & 1 & 1 & 1 & - & - & - & - \\
\hline
\end{tabular}

See table footnotes on page 61. 
TABLE A1. (Continued) Summary of classifications for hormonal contraceptive methods and intrauterine devices

\begin{tabular}{|c|c|c|c|c|c|c|c|c|c|}
\hline \multirow{2}{*}{$\begin{array}{l}\text { Condition } \\
\text { b. Not clinically well or not } \\
\text { receiving ARV therapy }\end{array}$} & \multicolumn{2}{|c|}{ Cu-IUD } & \multicolumn{2}{|c|}{ LNG-IUD } & \multirow{2}{*}{$\frac{\text { Implants }}{-}$} & \multirow{2}{*}{$\frac{\text { DMPA }}{-}$} & \multirow{2}{*}{$\frac{\text { POP }}{-}$} & \multirow{2}{*}{\multicolumn{2}{|c|}{$\frac{\mathrm{CHCs}}{-}$}} \\
\hline & 2 & 1 & 2 & 1 & & & & & \\
\hline \multicolumn{10}{|l|}{ Other Infections } \\
\hline \multicolumn{10}{|l|}{$\begin{array}{l}\text { Schistosomiasis } \\
\text { Schistosomiasis with fibrosis of } \\
\text { the liver is associated with } \\
\text { increased risk for adverse health } \\
\text { events as a result of pregnancy. }\end{array}$} \\
\hline a. Uncomplicated & & 1 & & 1 & 1 & 1 & 1 & & 1 \\
\hline $\begin{array}{l}\text { b. Fibrosis of the liver (if } \\
\text { severe, see Cirrhosis) }\end{array}$ & & 1 & & 1 & 1 & 1 & 1 & & 1 \\
\hline $\begin{array}{l}\text { Tuberculosis } \\
\text { This condition is associated with } \\
\text { increased risk for adverse health } \\
\text { events as a result of pregnancy. }\end{array}$ & Initiation & Continuation & Initiation & Continuation & & & & & \\
\hline a. Nonpelvic & 1 & 1 & 1 & 1 & $1^{*}$ & $1^{*}$ & $1^{*}$ & & $1^{*}$ \\
\hline b. Pelvic & 4 & 3 & 4 & 3 & $1^{*}$ & $1^{*}$ & $1^{*}$ & & $1^{*}$ \\
\hline Malaria & & 1 & & 1 & 1 & 1 & 1 & & 1 \\
\hline \multicolumn{10}{|l|}{$\begin{array}{l}\text { Diabetes } \\
\text { Insulin-dependent diabetes; } \\
\text { diabetes with nephropathy, } \\
\text { retinopathy, neuropathy, or } \\
\text { diabetes with other vascular } \\
\text { disease; or diabetes of }>20 \text { years' } \\
\text { duration are associated with } \\
\text { increased risk of adverse health } \\
\text { events as a result of pregnancy. }\end{array}$} \\
\hline $\begin{array}{l}\text { a. History of gestational disease } \\
\text { b. Nonvascular disease }\end{array}$ & & 1 & & 1 & 1 & 1 & 1 & & 1 \\
\hline i. Non-insulin dependent & & 1 & & 2 & 2 & 2 & 2 & & 2 \\
\hline ii. Insulin dependent & & 1 & & 2 & 2 & 2 & 2 & & 2 \\
\hline $\begin{array}{l}\text { c. Nephropathy, retinopathy, } \\
\text { or neuropathy }\end{array}$ & & 1 & & 2 & 2 & 3 & 2 & & $3 / 4^{*}$ \\
\hline $\begin{array}{l}\text { d. Other vascular disease or } \\
\text { diabetes of }>20 \text { years' duration }\end{array}$ & & 1 & & 2 & 2 & 3 & 2 & & $3 / 4^{*}$ \\
\hline \multicolumn{10}{|l|}{ Thyroid disorders } \\
\hline a. Simple goiter & & 1 & & 1 & 1 & 1 & 1 & & 1 \\
\hline b. Hyperthyroid & & 1 & & 1 & 1 & 1 & 1 & & 1 \\
\hline c. Hypothyroid & & 1 & & 1 & 1 & 1 & 1 & & 1 \\
\hline $\begin{array}{l}\text { Gastrointestinal Condition } \\
\text { Inflammatory bowel disease } \\
\text { (ulcerative colitis or Crohn's } \\
\text { disease) }\end{array}$ & & 1 & & 1 & 1 & 2 & 2 & & $2 / 3^{*}$ \\
\hline \multicolumn{10}{|l|}{$\begin{array}{l}\text { Gallbladder disease } \\
\text { a. Symptomatic }\end{array}$} \\
\hline $\begin{array}{l}\text { i. Treated by } \\
\text { cholecystectomy }\end{array}$ & & 1 & & 2 & 2 & 2 & 2 & & 2 \\
\hline ii. Medically treated & & 1 & & 2 & 2 & 2 & 2 & & 3 \\
\hline iii. Current & & 1 & & 2 & 2 & 2 & 2 & & 3 \\
\hline b. Asymptomatic & & 1 & & 2 & 2 & 2 & 2 & & 2 \\
\hline \multicolumn{10}{|l|}{ History of cholestasis } \\
\hline a. Pregnancy related & & 1 & & 1 & 1 & 1 & 1 & & 2 \\
\hline b. Past COC related & & 1 & & 2 & 2 & 2 & 2 & & 3 \\
\hline Viral hepatitis & & & & & & & & Initiation & n Continuation \\
\hline a. Acute or flare & & 1 & & 1 & 1 & 1 & 1 & $3 / 4^{*}$ & 2 \\
\hline b. Carrier & & 1 & & 1 & 1 & 1 & 1 & 1 & 1 \\
\hline c. Chronic & & 1 & & 1 & 1 & 1 & 1 & 1 & 1 \\
\hline \multicolumn{10}{|l|}{$\begin{array}{l}\text { Cirrhosis } \\
\text { Severe cirrhosis is associated } \\
\text { with increased risk for adverse } \\
\text { health events as a result of } \\
\text { pregnancy. }\end{array}$} \\
\hline a. Mild (compensated) & & 1 & & 1 & 1 & 1 & 1 & & 1 \\
\hline b. Severe (decompensated) & & 1 & & 3 & 3 & 3 & 3 & & 4 \\
\hline $\begin{array}{l}\text { Liver tumors } \\
\text { Hepatocellular adenoma and } \\
\text { malignant liver tumors are } \\
\text { associated with increased risk } \\
\text { for adverse health events as a } \\
\text { result of pregnancy. } \\
\text { a. Benign }\end{array}$ & & & & & & & & & \\
\hline
\end{tabular}

See table footnotes on page 61. 
TABLE A1. (Continued) Summary of classifications for hormonal contraceptive methods and intrauterine devices

\begin{tabular}{|c|c|c|c|c|c|c|c|c|}
\hline \multirow{4}{*}{$\begin{array}{l}\text { Condition } \\
\text { i. Focal nodular hyperplasia } \\
\text { ii. Hepatocellular adenoma } \\
\text { b. Malignant (hepatoma) }\end{array}$} & \multicolumn{2}{|c|}{ Cu-IUD } & \multicolumn{2}{|c|}{ LNG-IUD } & \multirow{2}{*}{$\frac{\text { Implants }}{2}$} & \multirow{2}{*}{$\frac{\text { DMPA }}{2}$} & \multirow{2}{*}{$\frac{P O P}{2}$} & \multirow{2}{*}{$\frac{\mathrm{CHCs}}{2}$} \\
\hline & & 1 & & 2 & & & & \\
\hline & & 1 & & 3 & 3 & 3 & 3 & 4 \\
\hline & & 1 & & 3 & 3 & 3 & 3 & 4 \\
\hline $\begin{array}{l}\text { Respiratory Conditions } \\
\text { Cystic fibrosis } \\
\text { This condition is associated with } \\
\text { increased risk for adverse health } \\
\text { events as a result of pregnancy. } \\
\text { Anemias }\end{array}$ & & $1^{*}$ & & $1^{*}$ & $1^{*}$ & $2^{*}$ & $1^{*}$ & $1^{*}$ \\
\hline Thalassemia & & 2 & & 1 & 1 & 1 & 1 & 1 \\
\hline $\begin{array}{l}\text { Sickle cell disease } \\
\text { This condition is associated with } \\
\text { increased risk for adverse health } \\
\text { events as a result of pregnancy. }\end{array}$ & & 2 & & 1 & 1 & 1 & 1 & 2 \\
\hline Iron-deficiency anemia & & 2 & & 1 & 1 & 1 & 1 & 1 \\
\hline $\begin{array}{l}\text { Solid Organ Transplantatio } \\
\text { Solid organ transplantation } \\
\text { This condition is associated with } \\
\text { increased risk for adverse health } \\
\text { events as a result of pregnancy. }\end{array}$ & on & Continuation & Initiation & Continuation & & & & \\
\hline $\begin{array}{l}\text { a. Complicated: graft failure } \\
\text { (acute or chronic), rejection, } \\
\text { or cardiac allograft } \\
\text { vasculopathy }\end{array}$ & 3 & 2 & 3 & 2 & 2 & 2 & 2 & 4 \\
\hline b. Uncomplicated & & 2 & & 2 & 2 & 2 & 2 & $2^{*}$ \\
\hline $\begin{array}{l}\text { Drug Interactions } \\
\text { Antiretroviral therapy } \\
\text { a. Nucleoside reverse } \\
\text { transcriptase inhibitors (NRTIs) }\end{array}$ & Initiation & Continuation & Initiation & Continuation & & & & \\
\hline i. Abacavir (ABC) & $1 / 2^{*}$ & $1 *$ & $1 / 2^{*}$ & $1^{*}$ & 1 & 1 & 1 & 1 \\
\hline ii. Tenofovir (TDF) & $1 / 2^{*}$ & $1^{*}$ & $1 / 2^{*}$ & $1^{*}$ & 1 & 1 & 1 & 1 \\
\hline iii. Zidovudine (AZT) & $1 / 2^{*}$ & $1^{*}$ & $1 / 2^{*}$ & $1^{*}$ & 1 & 1 & 1 & 1 \\
\hline iv. Lamivudine (3TC) & $1 / 2^{*}$ & $1^{*}$ & $1 / 2^{*}$ & $1^{*}$ & 1 & 1 & 1 & 1 \\
\hline v. Didanosine (DDI) & $1 / 2^{*}$ & $1^{*}$ & $1 / 2^{*}$ & $1^{*}$ & 1 & 1 & 1 & 1 \\
\hline vi. Emtricitabine (FTC) & $1 / 2^{*}$ & $1^{*}$ & $1 / 2^{*}$ & $1^{*}$ & 1 & 1 & 1 & 1 \\
\hline $\begin{array}{l}\text { vii. Stavudine (D4T) } \\
\text { b. Nonnucleoside reverse } \\
\text { transcriptase inhibitors } \\
\text { (NNRTIs) }\end{array}$ & $1 / 2^{*}$ & $1^{*}$ & $1 / 2^{*}$ & $1^{*}$ & 1 & 1 & 1 & 1 \\
\hline i. Efavirenz (EFV) & $1 / 2^{*}$ & $1^{*}$ & $1 / 2^{*}$ & $1^{*}$ & $2^{*}$ & $1^{*}$ & $2^{*}$ & $2^{*}$ \\
\hline ii. Etravirine (ETR) & $1 / 2^{*}$ & $1^{*}$ & $1 / 2^{*}$ & $1^{*}$ & 1 & 1 & 1 & 1 \\
\hline iii. Nevirapine (NVP) & $1 / 2^{*}$ & $1^{*}$ & $1 / 2^{*}$ & $1^{*}$ & 1 & 1 & 1 & 1 \\
\hline iv. Rilpivirine (RPV) & $1 / 2^{*}$ & $1^{*}$ & $1 / 2^{*}$ & $1^{*}$ & 1 & 1 & 1 & 1 \\
\hline $\begin{array}{l}\text { c. Ritonavir-boosted } \\
\text { protease inhibitors }\end{array}$ & & & & & & & & \\
\hline $\begin{array}{l}\text { i. Ritonavir-boosted } \\
\text { atazanavir (ATV/r) }\end{array}$ & $1 / 2^{*}$ & $1^{*}$ & $1 / 2^{*}$ & $1^{*}$ & $2^{*}$ & $1^{*}$ & $2^{*}$ & $2^{*}$ \\
\hline $\begin{array}{l}\text { ii. Ritonavir-boosted } \\
\text { darunavir (DRV/r) }\end{array}$ & $1 / 2^{*}$ & $1^{*}$ & $1 / 2^{*}$ & $1^{*}$ & $2^{*}$ & $1^{*}$ & $2^{*}$ & $2^{*}$ \\
\hline $\begin{array}{l}\text { iii. Ritonavir-boosted } \\
\text { fosemprenavir (FPV/r) }\end{array}$ & $1 / 2^{*}$ & $1^{*}$ & $1 / 2^{*}$ & $1^{*}$ & $2^{*}$ & $1^{*}$ & $2^{*}$ & $2^{*}$ \\
\hline $\begin{array}{l}\text { iv. Ritonavir-boosted } \\
\text { lopinavir (LPV/r) }\end{array}$ & $1 / 2^{*}$ & $1^{*}$ & $1 / 2^{*}$ & $1^{*}$ & 1 & 1 & 1 & 1 \\
\hline $\begin{array}{l}\text { v. Ritonavir-boosted } \\
\text { saquinavir (SQV/r) }\end{array}$ & $1 / 2^{*}$ & $1^{*}$ & $1 / 2^{*}$ & $1^{*}$ & $2^{*}$ & $1^{*}$ & $2^{*}$ & $2^{*}$ \\
\hline $\begin{array}{l}\text { vi. Ritonavir-boosted } \\
\text { tipranavir (TPV/r) } \\
\text { d. Protease inhibitors } \\
\text { without ritonavir }\end{array}$ & $1 / 2^{*}$ & $1^{*}$ & $1 / 2^{*}$ & $1^{*}$ & $2^{*}$ & $1^{*}$ & $2^{*}$ & $2^{*}$ \\
\hline i. Atazanavir (ATV) & $1 / 2^{*}$ & $1^{*}$ & $1 / 2^{*}$ & $1^{*}$ & 1 & 1 & 1 & $2^{*}$ \\
\hline ii. Fosamprenavir (FPV) & $1 / 2^{*}$ & $1^{*}$ & $1 / 2^{*}$ & $1^{*}$ & $2^{*}$ & $2^{*}$ & $2^{*}$ & $3^{*}$ \\
\hline iii. Indinavir (IDV) & $1 / 2^{*}$ & $1^{*}$ & $1 / 2^{*}$ & $1^{*}$ & 1 & 1 & 1 & 1 \\
\hline $\begin{array}{l}\text { iv. Nelfinavir (NFV) } \\
\text { e. CCR5 co-receptor } \\
\text { antagonists }\end{array}$ & $1 / 2^{*}$ & $1^{*}$ & $1 / 2^{*}$ & $1^{*}$ & $2^{*}$ & $1^{*}$ & $2^{*}$ & $2^{*}$ \\
\hline $\begin{array}{l}\text { i. Maraviroc (MVC) } \\
\text { f. HIV integrase strand } \\
\text { transfer inhibitors }\end{array}$ & $1 / 2^{*}$ & $1^{*}$ & $1 / 2^{*}$ & $1^{*}$ & 1 & 1 & 1 & 1 \\
\hline i. Raltegravir (RAL) & $1 / 2^{*}$ & $1^{*}$ & $1 / 2^{*}$ & $1^{*}$ & 1 & 1 & 1 & 1 \\
\hline ii. Dolutegravir (DTG) & $1 / 2^{*}$ & $1^{*}$ & $1 / 2^{*}$ & $1^{*}$ & 1 & 1 & 1 & 1 \\
\hline iii. Elvitegravir (EVG) & $1 / 2^{*}$ & $1^{*}$ & $1 / 2^{*}$ & $1^{*}$ & 1 & 1 & 1 & 1 \\
\hline
\end{tabular}

See table footnotes on next page. 
Recommendations and Reports

TABLE A1. (Continued) Summary of classifications for hormonal contraceptive methods and intrauterine devices

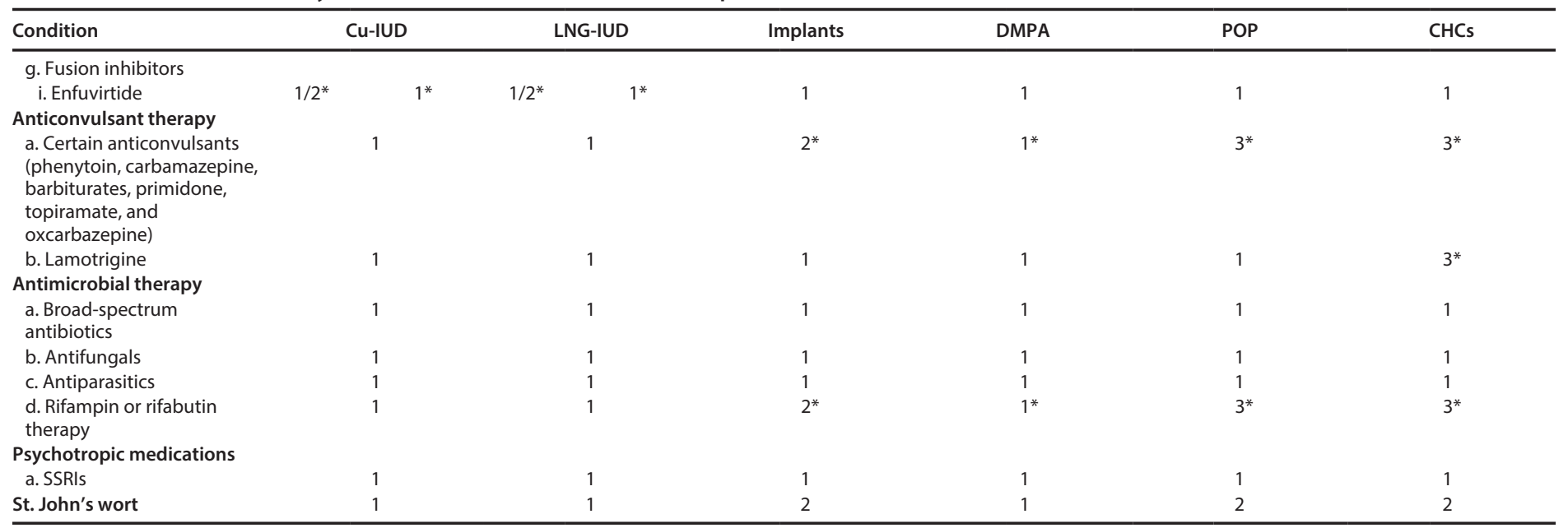

Abbreviations: $\mathrm{BMI}=$ body mass index; $\mathrm{COC}=$ combined oral contraceptive; $\mathrm{Cu}-\mathrm{IUD}=$ copper-containing IUD; $\mathrm{DMPA}=$ depot medroxyprogesterone acetate; $\mathrm{DV}=$ deep venous thrombosis; hCG = human chorionic gonadotropin; HDL = high-density lipoprotein; HIV = human immunodeficiency virus.; IUD = intrauterine device; $L D L=$ low-density lipoprotein; $L$ LG-IUD = levonorgestrel-releasing IUD; NA = not applicable; $\mathrm{PE}$ = pulmonary embolism; PID = pelvic inflammatory disease; $\mathrm{POP}$ = progestin-only pill; SSRI = selective serotonin reuptake inhibitor; STD = sexually transmitted disease.

* Consult the respective appendix for each contraceptive method in the 2016 U.S. Medical Eligibility Criteria for Contraceptive Use (1) for clarifications to the numeric categories.

\section{References}

1. Curtis KM, Tepper NK, Jatlaoui TC, et al. U.S. medical eligibility criteria for contraceptive use, 2016. MMWR Recomm Rep 2016;65(No. RR-3).
2. The Criteria Committee of the New York Heart Association. Nomenclature and criteria for diagnosis of diseases of the heart and great vessels. 9th ed. Boston, MA: Little, Brown \& Co; 1994. 


\section{Appendix B}

\section{When To Start Using Specific Contraceptive Methods}

\begin{tabular}{|c|c|c|c|}
\hline Contraceptive method & $\begin{array}{l}\text { When to start (if the provider is } \\
\text { reasonably certain that the woman } \\
\text { is not pregnant) }\end{array}$ & $\begin{array}{l}\text { Additional contraception } \\
\text { (i.e., back-up) needed }\end{array}$ & $\begin{array}{l}\text { Examinations or tests needed } \\
\text { before initiation* }\end{array}$ \\
\hline Copper-containing IUD & Anytime & Not needed & $\begin{array}{l}\text { Bimanual examination and cervical } \\
\text { inspection }^{\dagger}\end{array}$ \\
\hline Levonorgestrel-releasing IUD & Anytime & $\begin{array}{l}\text { If }>7 \text { days after menses started, use back-up } \\
\text { method or abstain for } 7 \text { days. }\end{array}$ & $\begin{array}{l}\text { Bimanual examination and cervical } \\
\text { inspection }^{\dagger}\end{array}$ \\
\hline Implant & Anytime & $\begin{array}{l}\text { If }>5 \text { days after menses started, use back-up } \\
\text { method or abstain for } 7 \text { days. }\end{array}$ & None \\
\hline Injectable & Anytime & $\begin{array}{l}\text { If }>7 \text { days after menses started, use back-up } \\
\text { method or abstain for } 7 \text { days. }\end{array}$ & None \\
\hline Combined hormonal contraceptive & Anytime & $\begin{array}{l}\text { If }>5 \text { days after menses started, use back-up } \\
\text { method or abstain for } 7 \text { days. }\end{array}$ & Blood pressure measurement \\
\hline Progestin-only pill & Anytime & $\begin{array}{l}\text { If }>5 \text { days after menses started, use back-up } \\
\text { method or abstain for } 2 \text { days. }\end{array}$ & None \\
\hline
\end{tabular}

Abbreviations: $\mathrm{BMI}=$ body mass index; HIV = human immunodeficiency virus; IUD = intrauterine device; STD = sexually transmitted disease; U.S. MEC = U.S. Medical Eligibility Criteria for Contraceptive Use.

* Weight (BMI) measurement is not needed to determine medical eligibility for any methods of contraception because all methods can be used (U.S. MEC 1) or generally can be used (U.S. MEC 2) among obese women (Box 1). However, measuring weight and calculating BMI (weight [kg] / height [m] ${ }^{2}$ ) at baseline might be helpful for monitoring any changes and counseling women who might be concerned about weight change perceived to be associated with their contraceptive method.

${ }^{\dagger}$ Most women do not require additional STD screening at the time of IUD insertion. If a woman with risk factors for STDs has not been screened for gonorrhea and chlamydia according to CDC's STD Treatment Guidelines (http://www.cdc.gov/std/treatment), screening can be performed at the time of IUD insertion, and insertion should not be delayed. Women with current purulent cervicitis or chlamydial infection or gonococcal infection should not undergo IUD insertion (U.S. MEC 4). 


\section{Appendix C}

\section{Examinations and Tests Needed Before Initiation of Contraceptive Methods}

The examinations or tests noted apply to women who are presumed to be healthy (Table C1). Those with known medical problems or other special conditions might need additional examinations or tests before being determined to be appropriate candidates for a particular method of contraception. The 2016 U.S. Medical Eligibility Criteria for Contraceptive Use (U.S. MEC) might be useful in such circumstances (1). The following classification was considered useful in differentiating the applicability of the various examinations or tests:

- Class A: essential and mandatory in all circumstances for safe and effective use of the contraceptive method.

- Class B: contributes substantially to safe and effective use, but implementation may be considered within the public health and/or service context; risk of not performing an examination or test should be balanced against the benefits of making the contraceptive method available.

- Class C: does not contribute substantially to safe and effective use of the contraceptive method.
These classifications focus on the relationship of the examinations or tests to safe initiation of a contraceptive method. They are not intended to address the appropriateness of these examinations or tests in other circumstances. For example, some of the examinations or tests that are not deemed necessary for safe and effective contraceptive use might be appropriate for good preventive health care or for diagnosing or assessing suspected medical conditions. Any additional screening needed for preventive health care can be performed at the time of contraception initiation and initiation should not be delayed for test results.

No examinations or tests are needed before initiating condoms or spermicides. A bimanual examination is necessary for diaphragm fitting. A bimanual examination and cervical inspection are needed for cervical cap fitting.

\section{References}

1. Curtis KM, Tepper NK, Jatlaoui TC, et al. U.S. medical eligibility criteria for contraceptive use, 2016. MMWR Recomm Rep 2016;65(No. RR-3).

TABLE C1. Examinations and tests needed before initiation of contraceptive methods

Contraceptive method and class

\begin{tabular}{|c|c|c|c|c|c|c|c|c|}
\hline \multirow[b]{2}{*}{ Examination or test } & \\
\hline & $\begin{array}{l}\text { Cu-IUD and } \\
\text { LNG-IUD }\end{array}$ & Implant & Injectable & $\mathrm{CHC}$ & POP & Condom & $\begin{array}{c}\text { Diaphragm } \\
\text { or } \\
\text { cervical cap }\end{array}$ & Spermicide \\
\hline \multicolumn{9}{|l|}{ Examination } \\
\hline Blood pressure & $\mathrm{C}$ & $\mathrm{C}$ & $\mathrm{C}$ & $A^{*}$ & $\mathrm{C}$ & $\mathrm{C}$ & C & $\mathrm{C}$ \\
\hline Weight (BMI) (weight $[\mathrm{kg}] /$ height $[\mathrm{m}]^{2}$ ) & $-十$ & $-\dagger$ & $-\dagger$ & $-{ }^{\dagger}$ & $-\dagger$ & $\mathrm{C}$ & C & $\mathrm{C}$ \\
\hline Clinical breast examination & C & $\mathrm{C}$ & C & $\mathrm{C}$ & C & $\mathrm{C}$ & C & $\mathrm{C}$ \\
\hline Bimanual examination and cervical inspection & A & $\mathrm{C}$ & C & $C$ & C & $\mathrm{C}$ & $A^{\S}$ & C \\
\hline \multicolumn{9}{|l|}{ Laboratory test } \\
\hline Glucose & C & $\mathrm{C}$ & C & C & C & $\mathrm{C}$ & C & C \\
\hline Lipids & $\mathrm{C}$ & $\mathrm{C}$ & C & $\mathrm{C}$ & C & $\mathrm{C}$ & C & $\mathrm{C}$ \\
\hline Liver enzymes & $\mathrm{C}$ & $\mathrm{C}$ & C & C & C & $\mathrm{C}$ & C & C \\
\hline Hemoglobin & $\mathrm{C}$ & $\mathrm{C}$ & C & C & C & $\mathrm{C}$ & C & C \\
\hline Thrombogenic mutations & $\mathrm{C}$ & $\mathrm{C}$ & C & $\mathrm{C}$ & C & $\mathrm{C}$ & C & $\mathrm{C}$ \\
\hline Cervical cytology (Papanicolaou test) & $\mathrm{C}$ & C & C & $\mathrm{C}$ & C & $\mathrm{C}$ & C & $\mathrm{C}$ \\
\hline STD screening with laboratory tests & -9 & C & C & C & C & $\mathrm{C}$ & C & $\mathrm{C}$ \\
\hline HIV screening with laboratory tests & $\mathrm{C}$ & C & C & C & C & $\mathrm{C}$ & C & $\mathrm{C}$ \\
\hline
\end{tabular}

Abbreviations: $\mathrm{BMI}=$ body mass index; $\mathrm{CHC}=$ combined hormonal contraceptive; $\mathrm{Cu}-\mathrm{IUD}=$ copper-containing intrauterine device; HIV = human immunodeficiency virus; LNG-IUD = levonorgestrel-releasing intrauterine device; POP = progestin-only pill; STD = sexually transmitted disease; U.S. MEC = U.S. Medical Eligibility Criteria for Contraceptive Use.

* In instances in which blood pressure cannot be measured by a provider, blood pressure measured in other settings can be reported by the woman to her provider.

+ Weight (BMI) measurement is not needed to determine medical eligibility for any methods of contraception because all methods can be used (U.S. MEC 1) or generally can be used (U.S. MEC 2) among obese women (Box 1). However, measuring weight and calculating BMl at baseline might be helpful for monitoring any changes and counseling women who might be concerned about weight change perceived to be associated with their contraceptive method.

$\S$ A bimanual examination (not cervical inspection) is needed for diaphragm fitting.

"Most women do not require additional STD screening at the time of IUD insertion. If a woman with risk factors for STDs has not been screened for gonorrhea and chlamydia according to CDC's STD Treatment Guidelines (http://www.cdc.gov/std/treatment), screening can be performed at the time of IUD insertion, and insertion should not be delayed. Women with current purulent cervicitis or chlamydial infection or gonococcal infection should not undergo IUD insertion (U.S. MEC 4). 


\section{Appendix D}

\section{Routine Follow-Up After Contraceptive Initiation}

These recommendations address when routine follow-up is recommended for safe and effective continued use of contraception for healthy women (Table D1). The recommendations refer to general situations and might vary for different users and different situations. Specific populations who might benefit from frequent follow-up visits include adolescents, those with certain medical conditions or characteristics, and those with multiple medical conditions.

TABLE D1. Routine follow-up after contraceptive initiation

\begin{tabular}{|c|c|c|c|c|c|}
\hline \multirow[b]{2}{*}{ Action } & \multicolumn{5}{|c|}{ Contraceptive method } \\
\hline & CU-IUD or LNG-IUD & Implant & Injectable & $\mathrm{CHC}$ & POP \\
\hline \multicolumn{6}{|l|}{ General follow-up } \\
\hline $\begin{array}{l}\text { Advise women to return at any time to discuss side effects or } \\
\text { other problems or if they want to change the method. Advise } \\
\text { women using IUDs, implants, or injectables when the IUD or } \\
\text { implant needs to be removed or when a reinjection is needed. } \\
\text { No routine follow-up visit is required. }\end{array}$ & $x$ & $x$ & $x$ & $x$ & $x$ \\
\hline \multicolumn{6}{|l|}{ Other routine visits } \\
\hline $\begin{array}{l}\text { Assess the woman's satisfaction with her current method and } \\
\text { whether she has any concerns about method use. }\end{array}$ & $x$ & $x$ & $x$ & $x$ & $x$ \\
\hline $\begin{array}{l}\text { Assess any changes in health status, including medications, that } \\
\text { would change the method's appropriateness for safe and } \\
\text { effective continued use based on U.S. MEC (i.e., category } 3 \text { and } 4 \\
\text { conditions and characteristics) (Box 1). }\end{array}$ & $\mathrm{x}$ & $x$ & $x$ & $\mathrm{x}$ & $x$ \\
\hline $\begin{array}{l}\text { Consider performing an examination to check for the presence } \\
\text { of IUD strings. }\end{array}$ & $\mathrm{x}$ & - & - & - & - \\
\hline $\begin{array}{l}\text { Consider assessing weight changes and counseling women } \\
\text { who are concerned about weight change perceived to be } \\
\text { associated with their contraceptive method. }\end{array}$ & $x$ & $\mathrm{x}$ & $x$ & $x$ & $x$ \\
\hline Measure blood pressure. & - & - & - & $x$ & - \\
\hline
\end{tabular}

Abbreviations: $\mathrm{CHC}=$ combined hormonal contraceptives; $\mathrm{Cu}-\mathrm{IUD}=$ copper-containing intrauterine device; $\mathrm{HIV}=$ human immunodeficiency virus; IUD = intrauterine device; LNG-IUD = levonorgestrel-releasing intrauterine device; POP = progestin-only pills; U.S. MEC = U.S. Medical Eligibility Criteria for Contraceptive Use. 


\section{Appendix E}

\section{Management of Women with Bleeding Irregularities While Using Contraception*}

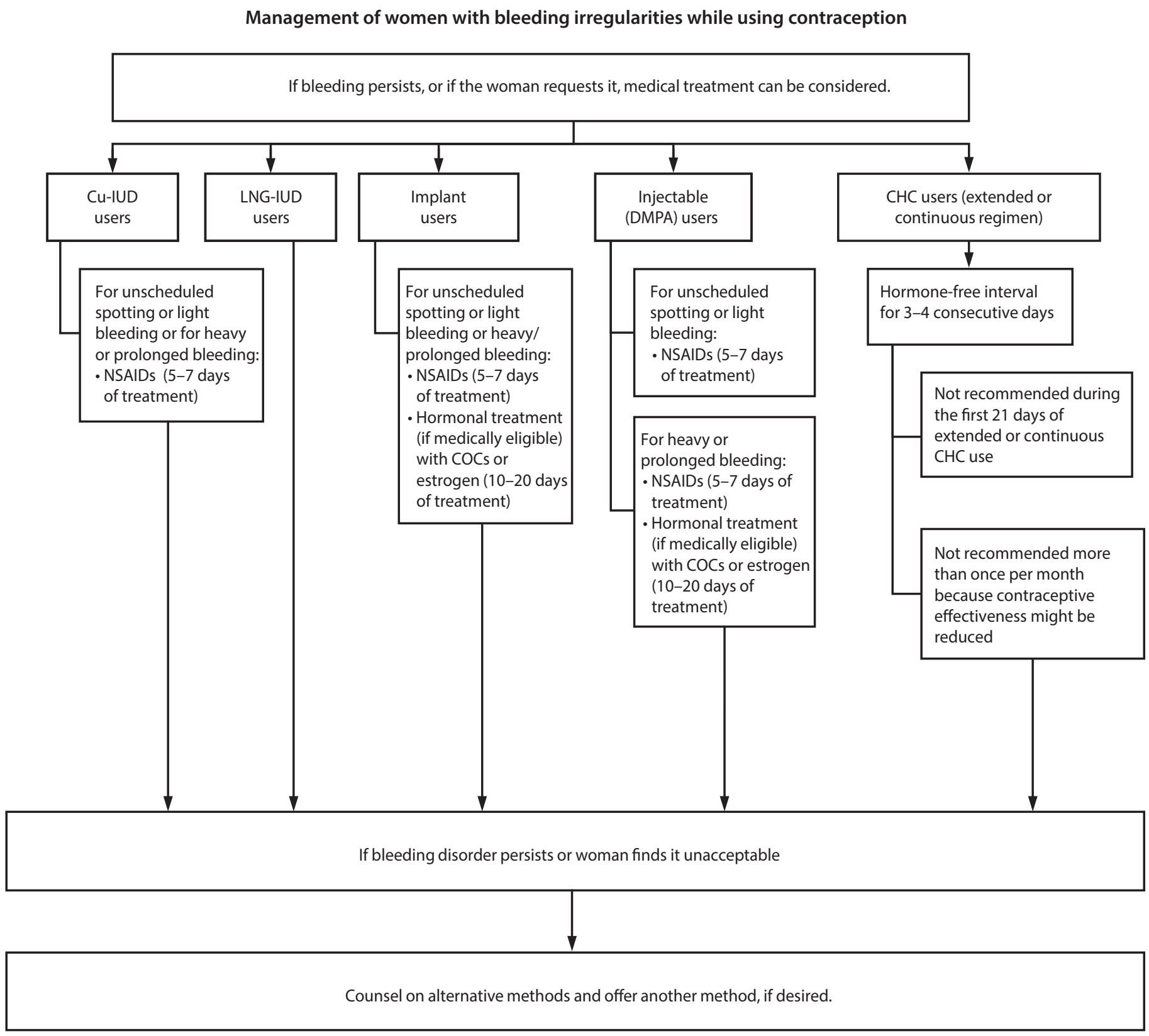

Abbreviations: $\mathrm{CHC}=$ combined hormonal contraceptive; $\mathrm{COC}=$ combined oral contraceptive; $\mathrm{Cu}-\mathrm{IUD}=$ copper-containing intrauterine device; DMPA $=$ depot medroxyprogesterone acetate; LNG-IUD = levonorgestrel-releasing intrauterine device; NSAIDs = nonsteroidal antiinflammatory drugs.

* If clinically warranted, evaluate for underlying condition. Treat the condition or refer for care. Heavy or prolonged bleeding, either unscheduled or menstrual, is uncommon among LNG-IUD users and implant users. 


\section{Appendix F}

\section{Management of Intrauterine Devices When Users are Found To Have Pelvic Inflammatory Disease*}

Management of intrauterine devices when users of copper-containing intrauterine devices or levonorgestrel-releasing intrauterine devices are found to have pelvic inflammatory disease

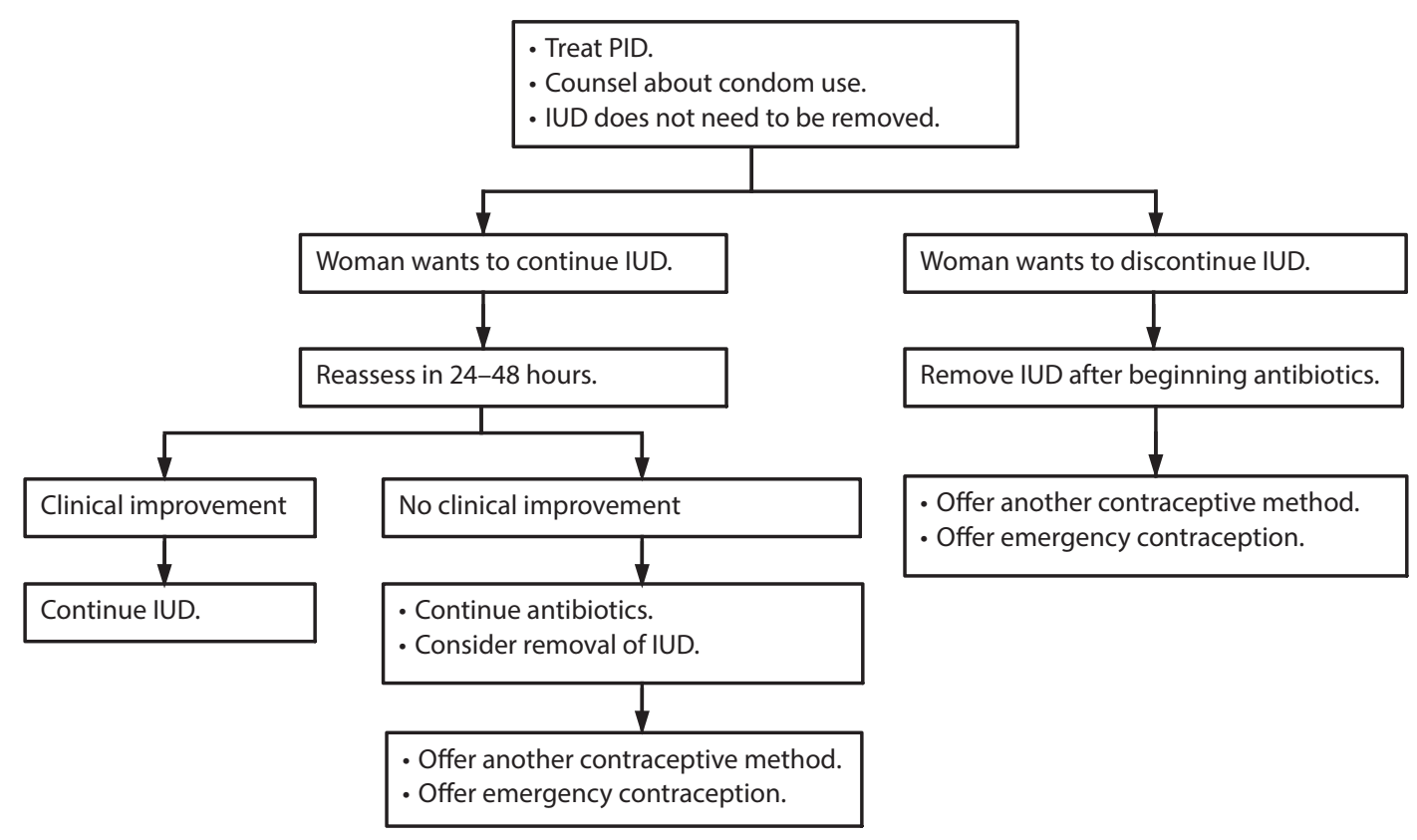

Abbreviations: IUD = intrauterine device; PID = pelvic inflammatory disease.

* Treat according to the CDC Sexually Transmitted Diseases Treatment Guidelines (http://www.cdc.gov/std/treatment). 



The Morbidity and Mortality Weekly Report (MMWR) Series is prepared by the Centers for Disease Control and Prevention (CDC) and is available free of charge in electronic format. To receive an electronic copy each week, visit MMWR's free subscription page at http://www.cdc.gov/mmwr/mmwrsubscribe.html. Paper copy subscriptions are available through the Superintendent of Documents, U.S. Government Printing Office, Washington, DC 20402; telephone 202-512-1800.

Readers who have difficulty accessing this PDF file may access the HTML file at http://www.cdc.gov/mmwr/volumes/65/rr/rr6504a1.htm?s_cid=rr6504a1_w. Address all inquiries about the $M M W R$ Series, including material to be considered for publication, to Executive Editor, MMWR Series, Mailstop E-90, CDC, 1600 Clifton Rd., N.E., Atlanta, GA 30329-4027 or to mmwrq@cdc.gov.

All material in the MMWR Series is in the public domain and may be used and reprinted without permission; citation as to source, however, is appreciated. Use of trade names and commercial sources is for identification only and does not imply endorsement by the U.S. Department of Health and Human Services.

References to non-CDC sites on the Internet are provided as a service to $M M W R$ readers and do not constitute or imply endorsement of these organizations or their programs by CDC or the U.S. Department of Health and Human Services. CDC is not responsible for the content of these sites. URL addresses listed in $M M W R$ were current as of the date of publication.

ISSN: $1057-5987$ (Print) 